\title{
AJUSTES PARA A ESTATÍSTICA DA RAZÃO DE VEROSSIMILHANÇAS SINALIZADA EM MODELOS NORMAIS NÃO LINEARES
}

\author{
Daniela Carine Ramires
}

\author{
Dissertaça Apresentada aO \\ Instituto dE MATEMÁtICA E Estatística \\ DA UNIVTRSIDADE DE SÃO PAULO \\ para Obtenção do Grau de \\ Mestre eM Estatística
}

Area de Concentração: Estatística

Orientadora: Profa. Dra. Silvia Lopes de Paula Ferrari

\author{
Durante a elaboragáo deste trabalho a autora \\ recebeu apoio financiro parcial do $C N P q$
}




\title{
AJUSTES PARA A \\ ESTATÍSTICA DA RAZÃO DE \\ VEROSSIMILHANÇAS SINALIZADA \\ EM MODELOS NORMAIS \\ NÃO LINEARES
}

\author{
Este exemplar corresponde à redação final \\ da dissertação devidamente corrigida e \\ defendida por Daniela Carine Ramires \\ e aprovada pela comissão julgadora.
}

São Paulo, 20 de setembro de 2004.

Banca examinadora:

- Profa. Dra. Silvia Lopes de Paula Ferrari (Orientadora) - IME - USP

- Profa. Dra. Denise Aparecida Botter - IME - USP

- Profa. Dra. Reiko Aoki - ICMC - USP 
A Deus,

que me deu força

em todos os momentos,

DEDICO 


\section{Agradecimentos}

* Aos meus pais, Odair e Maria Luiza e às minhas irmãs Alessandra, Fabiana e Luciana, que mesmo distantes de mim, me incentivaram, me deram força e amor, sem os quais eu não teria conseguido concluir esta etapa tão importante.

* Ao meu namorado Marcos, que teve uma participação muito importante neste trabalho, me ajudando nos momentos mais difíceis e também em minha vida, contribuindo com seu amor, carinho e paciência.

* À professora Silvia Lopes de Paula Ferrari, pela orientação competente, pela confiança depositada na realização desse trabalho, pelos ensinamentos técnicos, pela ajuda e compreensão que muito contribuíram para meu amadurecimento profissional.

* A todos os professores da graduação em estatística da Universidade Estadual Paulista por fornecerem bagagem teórica e prática que contribuiu para o meu mestrado em estatística. Em especial, ao professor Sergio Minoru Oikawa, que me orientou na iniciação científica e contribuiu com seus ensinamentos técnicos, lições de perseverança, estímulo, conselhos e amizade.

* Aos professores do Departamento de Estatística do IME-USP, pelos valiosos ensinamentos recebidos ao longo do mestrado e, em especial, ao Prof. Gilberto 
A. Paula por diversas oportunidades que me proporcionou durante o mestrado e ao Prof. Carlos Alberto B. Pereira que me orientou em meu primeiro artigo.

* À Universidade de São Paulo e ao CNPq, por me concederem a oportunidade de cursar o Mestrado e pelos recursos disponibilizados.

* À Universidade Metodista de São Paulo por me empregar como professora de estatística nos cursos de ciência da computação, engenharia da computação, matemática e administração geral.

* Aos meus amigos Cléber e Lourdes e aos meus amigos do trabalho, que me auxiliaram direta ou indiretamente durante a execução desse trabalho, fornecendo suas possíveis colaborações nas horas em que mais precisei. 


\section{Resumo}

A estatística da razão de verossimilhanças sinalizada $(R)$ tem, assintoticamente, distribuição normal padrão com um erro de ordem $O\left(n^{-1 / 2}\right)$. Em amostras pequenas ou de tamanho moderado, a aproximação normal para a distribuição de $R$ pode ser bem imprecisa. Diversos ajustes para esta estatística são apresentados na literatura, entre os quais, destacamos a estatística da razão de verossimilhanças sinalizada modificada $\left(R^{*}\right)$ proposta por Barndorff-Nielsen $(1986,1991)$, a estatística $R_{0}^{*}$ proposta por DiCiccio e Martin (1993) para o caso em que os parâmetros são ortogonais, a estatística $\bar{R}^{*}$ baseada em covariâncias proposta por Skovgaard (1996), a estatística $\widehat{R}^{*}$ baseada em covariâncias empíricas proposta por Severini (1999) e, finalmente, a estatística $\widetilde{R}^{*}$ baseada numa estatística aproximadamente ancilar proposta por Fraser, Reid e Wu (1999). Nessa dissertação obtivemos estas estatísticas para os modelos de regressão normais lineares e não lineares e, através de um estudo de simulação, fizemos uma comparação dos desempenhos dos testes baseados nas diversas estatísticas consideradas. Por fim, aplicamos os resultados teóricos a dados reais. 


\section{Abstract}

The signed likelihood ratio statistic $(R)$ is asymptotically distributed according to a standard normal distribution up to an error of order $O\left(n^{-1 / 2}\right)$. If the sample size is small, the normal approximation to the distribution of $R$ may be very inaccurate. A number of adjusted statistics with better finite sample properties are presented in the statistical literature. In this thesis we obtain some adjusted signed likelihood ratio statistics in normal linear and nonlinear regression models, namely: the statistic $R^{*}$ proposed by Barndorff-Nielsen $(1986,1991)$, the statistic $R_{0}^{*}$ obtained by DiCiccio and Martin (1993) using an orthogonal parameterization, the statistic $\bar{R}^{*}$ based on covariances (Skovgaard, 1996), the statistic $\widehat{R}^{*}$ based on empirical covariances (Severini, 1999) and the statistic $\widetilde{R}^{*}$ based on an approximately ancillary statistic obtained by Fraser, Reid and Wu (1999). We compare the performance of the tests that use these statistics coupled with the normal approximation using Monte Carlo simulated samples. Finally, we apply the theoretical results in real data sets. 


\section{Índice}

1 Introdução

2 Modelos Normais Não Lineares 4

2.1 Introdução . . . . . . . . . . . . . . . . . . 4

2.2 Estimação dos Parâmetros . . . . . . . . . . . . . . . . . . 6

2.3 Estatística da Razão de Verossimilhanças Sinalizada $(R) \ldots \ldots$

3 Ajustes para a Estatística da Razão de Verossimilhanças Sinalizada 16

3.1 Notação . . . . . . . . . . . . . . . . . . . . . . . . . 16

3.2 Estatística da Razão de Verossimilhanças Sinalizada Modificada $\left(R^{*}\right) \quad 17$

3.3 Aproximações para $R^{*} \ldots \ldots \ldots \ldots$. . . . . . . . . . . . 21

3.3 .1 Introdução . . . . . . . . . . . . . . . . . . . . . 21

3.3.2 Estatística da Razão de Verossimilhanças Sinalizada Modificada baseada em Parâmetros Ortogonais $\left(R_{0}^{*}\right) \ldots \ldots . . .22$

viii 
Índice

3.3.3 Estatística da Razão de Verossimilhanças Sinalizada Modificada baseada em Covariâncias $\left(\bar{R}^{*}\right) \ldots \ldots \ldots . \ldots 23$

3.3.4 Estatística da Razão de Verossimilhanças Sinalizada Modifcada baseada em Covariâncias Empíricas $\left(\widehat{R}^{*}\right) \ldots \ldots \ldots$

3.3.5 Estatística da Razão de Verossimilhanças Sinalizada Modificada baseada numa Estatística Aproximadamente Ancilar $\left(\widetilde{R}^{*}\right) \quad 28$

4 Estudos de Simulação $\quad 32$

4.1 Introdução . . . . . . . . . . . . . . . . . . . 32

4.2 Detalhes Metodológicos . . . . . . . . . . . . . . . . . . 33

4.3 Tamanho dos Testes . . . . . . . . . . . . . . . . 34

4.4 Poder dos Testes . . . . . . . . . . . . . . . . . 51

5 Aplicação a Dados Reais $\quad 60$

5.1 Câncer de Mama . . . . . . . . . . . . . . . . . . . 60

5.2 Crescimento de Feijoeiros . . . . . . . . . . . . . . 64

$\begin{array}{lll}6 & \text { Conclusões } & 67\end{array}$

$\begin{array}{ll}\text { A Programas } & 69\end{array}$

$\begin{array}{ll}\text { Referências Bibliográficas } & 74\end{array}$ 


\title{
Lista de Símbolos
}

\author{
$\psi \quad$ parâmetro escalar de interesse \\ $\lambda \quad$ vetor de parâmetros de perturbação $\left(\beta^{\top}, \sigma\right)^{\top}$ de dimensão $s \times 1$ \\ $\beta \quad$ vetor de parâmetros de dimensão $p \times 1$ \\ $\sigma \quad$ parâmetro escalar \\ $\theta \quad$ vetor de parâmetros $\left(\psi, \beta^{\top}, \sigma\right)^{\top}$ de dimensão $(s+1) \times 1$ \\ $\nu \quad$ vetor de parâmetros $\left(\psi, \beta^{\top}\right)^{\top}$ de dimensão $s \times 1$ \\ $e \quad$ vetor coluna $n \times 1$ de uns \\ 0 vetor ou matriz de zeros de dimensão adequada \\ $L(\theta)$ função de verossimilhança \\ $l(\theta) \quad$ logaritmo natural da função de verossimilhança \\ $\widehat{\theta} \quad$ estimador de máxima verossimilhança de $\theta$ \\ $\widehat{\psi} \quad$ estimador de máxima verossimilhança de $\psi$ \\ $\hat{\lambda} \quad$ estimador de máxima verossimilhança de $\lambda$ \\ $\widehat{\lambda}_{\psi} \quad$ estimador de máxima verossimilhança de $\lambda$ quando $\psi$ é fixado \\ $l_{\theta}(\theta) \quad \partial l(\theta) / \partial \theta$ \\ $l(\theta ; \hat{\theta}, a)$ logaritmo natural da função de verossimilhança escrito como \\ função de $\theta, \widehat{\theta}$ e, se necessário, uma estatística ancilar $a$ tal \\ que $(\widehat{\theta}, a)$ seja uma estatística suficiente
}


Lista de Símbolos

$$
\begin{array}{ll}
l_{; \widehat{\psi}}(\theta) & \partial l(\theta ; \widehat{\theta}, a) / \partial \widehat{\psi} \\
l_{\widehat{\lambda}^{\top}}(\theta) & \partial l(\theta ; \widehat{\theta}, a) / \partial \widehat{\lambda}^{\top} \\
l_{\lambda ; \widehat{\psi}}(\theta) & \partial^{2} l(\theta ; \widehat{\theta}, a) / \partial \lambda \partial \widehat{\psi} \\
l_{\theta \theta}(\theta) & \partial^{2} l(\theta) / \partial \theta \partial \theta^{\top} \\
l_{\widehat{\theta} \hat{\theta}}(\theta) & \partial^{2} l(\theta) / \partial \widehat{\theta} \partial \widehat{\theta^{\top}} \\
j(\theta) & -l_{\theta \theta}(\theta) ; \text { informação observada } \\
j_{\lambda \lambda}(\theta) & -l_{\lambda \lambda}(\theta) \\
i\left(\theta, \theta_{0}\right) & -E_{\theta_{0}}\left[l_{\theta}(\theta) l_{\theta}\left(\theta_{0}\right)^{\top}\right] \\
i_{\psi \psi}\left(\theta, \theta_{0}\right) & -E_{\theta_{0}}\left[l_{\psi}(\theta) l_{\psi}\left(\theta_{0}\right)^{\top}\right] \\
i(\theta) & i(\theta, \theta) ; \text { informação esperada } \\
|A| & \text { determinante de A } \\
R & \text { estatística da razão de verossimilhanças sinalizada } \\
R^{*} & \text { estatística da razão de verossimilhanças sinalizada modificada } \\
R_{0}^{*} & \text { estatística da razão de verossimilhanças sinalizada modificada } \\
& \text { baseada em parâmetros ortogonais } \\
\widetilde{R}^{*} & \text { estatística da razão de verossimilhanças sinalizada modificada } \\
\widetilde{R}^{*} & \text { baseada em covariâncias } \\
& \text { estatística da razão de verossimilhanças sinalizada modificada } \\
& \text { baseada numa estatística aproximadamente ancilar }
\end{array}
$$




\section{Capítulo 1}

\section{Introdução}

Um modelo estatístico paramétrico é uma coleção de distribuições de probabilidade indexadas por um vetor de parâmetros $\theta$ desconhecido, esse pertencente ao conjunto $\Theta$, o espaço paramétrico. Considere $\theta=\left(\psi, \lambda^{\top}\right)^{\top}$, onde $\psi$ é um parâmetro escalar e $\lambda$ é um parâmetro vetorial de dimensão $s$. Suponha ainda que $\psi$ seja o parâmetro de interesse primário do pesquisador enquanto que $\lambda$ é o de interesse secundário e será aqui chamado de parâmetro de perturbação.

Nessa dissertação, nosso foco estará em testes de hipóteses unilaterais sobre $\psi$. A hipótese nula $\mathrm{H}_{0}: \psi \geq \psi_{0}$ será testada contra a hipótese alternativa $\mathrm{H}_{1}: \psi<\psi_{0}$ ou ainda essas hipóteses serão especificadas como $\mathrm{H}_{0}: \psi \leq \psi_{0}$ e $\mathrm{H}_{1}: \psi>\psi_{0}$. Nessas situações, é usual a utilização da estatística da razão de verossimilhanças sinalizada, que denotaremos por $R$.

Assintoticamente, a estatística $R$ tem distribuição normal padrão com um erro de ordem $O\left(n^{-\frac{1}{2}}\right)$. Para amostras pequenas ou de tamanho moderado esta aproximação pode ser ruim. Com o intuito de melhorar a qualidade da aproximação da estatística $R$ pela distribuição normal padrão, vários ajustes foram propostos na literatura. Severini (2000, Capítulo 7) faz uma revisão bibliográfica sobre tais 
ajustes. A idéia original de ajustar a estatística da razão de verossimilhanças sinalizada foi de Barndorff-Nielsen (1986). Posteriormente foi tratada por Fraser (1990), novamente por Barndorff-Nielsen $(1991,1995)$ e por Jensen $(1992,1993,1997)$. Discussões gerais sobre as diversas formas ajustadas para $R$ e suas propriedades são dadas em Barndorff-Nielsen e Cox (1994), Jensen (1995), Reid (1996) e Skovgaard (2001). As propriedades assintóticas desses ajustes foram estudadas por alguns autores, tais como Barndorff-Nielsen (1986, 1990, 1991, 1995), Jensen (1995), Sweeting (1995) e Skovgaard $(1996,2001)$. Uma avaliação recente de dois desses ajustes pode ser encontrada em Larsen, Blæsild e Sørensen (2002).

Um dos objetivos desse trabalho é apresentar a estatística da razão de verossimilhanças sinalizada modificada $\left(R^{*}\right)$ proposta por Barndorff-Nielsen $(1986,1991)$ e as seguintes aproximações para $R^{*}$ : a estatística $R_{0}^{*}$ proposta por DiCiccio e Martin (1993) para o caso em que os parâmetros são ortogonais, a estatística $\bar{R}^{*}$ baseada em covariâncias proposta por Skovgaard (1996), a estatística $\widehat{R}^{*}$ baseada em covariâncias empíricas proposta por Severini (1999) e, finalmente, a estatística $\widetilde{R}^{*}$ baseada numa estatística aproximadamente ancilar proposta por Fraser, Reid e Wu (1999). Os outros objetivos são aplicá-las em modelos de regressão normais lineares e não lineares, realizar um estudo de simulação para comparar os desempenhos dos testes baseados nas diversas estatísticas ajustadas e, por fim, aplicar os resultados teóricos a conjuntos de dados reais.

Essa dissertação é constituída por seis capítulos. No segundo capítulo, é apresentado o modelo de regressão normal não linear, estimação dos parâmetros e a estatística da razão de verossimilhanças sinalizada $(R)$. No terceiro capítulo são apresentadas seis estatísticas da razão de verossimilhanças sinalizadas ajustadas propostas na literatura. No quarto capítulo são avaliados os comportamentos dos testes baseados nessas estatísticas com relação a tamanho e poder, sob diversas situações, por meio 
de simulações de Monte Carlo. Procuraremos identificar os testes que tenham tamanhos empíricos próximos aos nominais e, ao mesmo tempo, tenham maior poder. No quinto capítulo, são feitas aplicações dos testes a dois conjuntos de dados reais. Finalmente, no sexto capítulo, são apresentadas as conclusões. 


\section{Modelos Normais Não Lineares}

\subsection{Introdução}

O modelo de regressão normal não linear tem sido utilizado em diversas áreas há diversas décadas. A principal característica desses modelos é que, em geral, são deduzidos a partir de suposições teóricas (quase sempre equações diferenciais) e os parâmetros resultantes são interpretáveis.

A forma clássica do modelo normal não linear é dada considerando $Y_{1}, Y_{2}, \ldots, Y_{n}$ variáveis aleatórias tais que

$$
Y_{i}=g_{i}(\nu)+\sigma \varepsilon_{i}, \quad i=1, \ldots, n
$$

em que $g_{i}(\nu)=g_{i}\left(\nu, x_{i}\right)$ é uma função contínua conhecida, diferenciável em $\nu$, possivelmente não linear, com $\nu=\left(\psi, \beta^{\top}\right)^{\top}$ um vetor de dimensão $s=p+1$ de parâmetros desconhecidos a serem estimados, em que $\psi$ é o de interesse, $\psi \in \mathbb{R}$, $\beta$ é um vetor de dimensão $p, \beta \in \mathbb{R}^{p}, x_{i}$ é um vetor coluna de dimensão $s$ de covariadas conhecidas, $\sigma>0$ é um parâmetro escalar desconhecido e os $\varepsilon_{i}^{\prime} s$ são variáveis aleatórias independentes, não observáveis, com distribuição normal padrão. O parâmetro de perturbação, conforme mencionado no Capítulo 1 , é $\lambda=\left(\beta^{\top}, \sigma\right)^{\top} \mathrm{e}$ 
a função densidade de probabilidade de $Y_{i}$ é

$$
f\left(y_{i}, \theta\right)=\frac{1}{\sqrt{2 \pi} \sigma} \exp \left\{-\frac{\left[y_{i}-g_{i}(\nu)\right]^{2}}{2 \sigma^{2}}\right\}, \quad y_{i} \in \mathbb{R}
$$

em que $\theta=\left(\psi, \beta^{\top}, \sigma\right)^{\top}$.

A seguir apresentamos alguns exemplos de estruturas linear e não lineares para a média de $Y_{i}, \mathrm{E}\left(Y_{i}\right)=g_{i}(\nu)$, e no Capítulo 5 daremos maiores detalhes de suas possíveis aplicações. As estruturas não lineares, interpretação dos parâmetros e aplicações apresentadas a seguir foram extraídas do artigo de Yi, Wu e Liu (2002) e de Cordeiro e Paula (1989).

Uma possível estrutura para a média de $Y_{i}$ é

$$
g_{i}(\nu)=x_{i}^{\top} \nu, \quad i=1,2, \ldots, n
$$

com $\nu=\left(\nu_{1}, \nu_{2}, \ldots, \nu_{s}\right)^{\top}, \nu_{i} \in \mathbb{R}$, para $i=1,2, \ldots, s$ e $x_{i}=\left(x_{i 1}, x_{i 2}, \ldots, x_{i s}\right)^{\top}$, ou seja, a relação entre a $\mathrm{E}\left(Y_{i}\right)$ e os parâmetros $\nu_{1}, \nu_{2}, \ldots, \nu_{s}$ é linear.

Em aplicações de modelos normais não lineares, Yi, Wu e Liu (2002) trazem como exemplo a função não linear, média de $Y_{i}$, dada por

$$
g_{i}(\nu)=\frac{\nu_{1} x_{i}}{\nu_{2}+x_{i}}, \quad i=1,2, \ldots, 10
$$

em que $\nu_{i} \geq 0$, para $i=1,2$. Esta função é conhecida como Modelo de MichaelisMenten. Yi, Wu e Liu (2002) apresentam uma aplicação do modelo (2.1) com esta função (2.4) em um estudo do câncer de mama em mulheres. Nesse estudo, a variável $x_{i}$ é a quantidade de estrógeno receptor contido no tumor e $Y_{i}$ representa o resultado do tratamento através da terapia endócrina. O parâmetro de interesse a ser testado é, em geral, $\psi=\nu_{2}$.

Um fato interessante no modelo de Michaelis-Menten é que $\lim _{x_{i} \rightarrow+\infty} g_{i}(\nu)=$ $\lim _{x_{i} \rightarrow+\infty} g_{i}\left(\nu, x_{i}\right)=\nu_{1}$. Assim, o parâmetro $\nu_{1}$ pode ser interpretado como uma 
assíntota horizontal. Nessa aplicação, como $\nu_{1} \geq 0$, o parâmetro $\nu_{2}$ está relacionado com a taxa média de decrescimento da curva.

Cordeiro e Paula (1989, p.189) ilustram um modelo não linear geralmente aplicado na área de agricultura para explicar o comprimento de diversos tipos de feijoeiros $\left(Y_{i}\right)$ em função da quantidade de água na raíz $\left(x_{i}\right)$. O modelo apresentado pelos autores é o modelo (2.1) com

$$
g_{i}(\nu)=\nu_{1} \exp \left\{-\exp \left(\nu_{2}-\nu_{3} x_{i}\right)\right\} \quad i=1,2, \ldots, 15
$$

em que, $\nu_{1}>0, \nu_{2}$ e $\nu_{3} \in \mathbb{R}$. Esse modelo normal não linear é conhecido como modelo de Gompertz.

No modelo de Gompertz, o parâmetro de interesse é, em geral, $\psi=\nu_{3}$. Note que se $\nu_{3}>0, \lim _{x_{i} \rightarrow+\infty} g_{i}(\nu)=\lim _{x_{i} \rightarrow+\infty} g_{i}\left(\nu, x_{i}\right)=\nu_{1}$ e, sendo assim, o parâmetro $\nu_{1}$ pode ser interpretado como assíntota horizontal. O parâmetro $\nu_{2}$ está relacionado com o intercepto, isto é, com o valor de $g_{i}(\nu)=E\left(Y_{i}\right)$ correspondente a $x_{i}=0$, no sentido de que, dado o valor de $\nu_{1}$, o intercepto pode ser determinado conhecendo-se $\nu_{2}$. O parâmetro $\nu_{3}$ está relacionado com a taxa média de decrescimento da curva.

\subsection{Estimação dos Parâmetros}

Considere o modelo normal não linear dado em (2.1), ou seja, admita que $Y_{i} \sim$ $\mathrm{N}\left(g_{i}(\nu), \sigma^{2}\right)$ e que $Y_{1}, Y_{2}, \ldots, Y_{n}$ são variáveis aleatórias independentes. A função de densidade conjunta vista como função de $\theta=\left(\psi, \beta^{\top}, \sigma\right)^{\top}$,

$$
L(\theta)=L(\theta ; y)=\prod_{i=1}^{n} f\left(y_{i} ; \theta\right)
$$


é denominada função de verossimilhança de $\theta$ correspondente à amostra observada $y=\left(y_{1}, y_{2}, \ldots, y_{n}\right)^{\top}$. Em modelos normais não lineares, temos

$$
L(\theta)=\sigma^{-n} \exp \left\{-\sum_{i=1}^{n} \frac{\left[y_{i}-g_{i}(\nu)\right]^{2}}{2 \sigma^{2}}\right\} .
$$

Note que na função $L(\theta)$, o fator $(2 \pi)^{-\frac{n}{2}}$ foi omitido, pois não depende de $\theta$.

A verossimilhança é interpretada como função do vetor de parâmetros $(\theta)$ para um conjunto de dados fixo $(y)$. Dizemos que um conjunto de dados é mais consistente com um vetor $\theta_{1}$ do que com $\theta_{2}$ se a verossimilhança associada a $\theta_{1}$ for maior do que a associada a $\theta_{2}$. Generalizando, entre os possíveis candidatos para estimar o parâmetro verdadeiro (digamos $\theta_{0}=\left(\psi_{0}, \beta_{0}^{\top}, \sigma_{0}\right)^{\top}$ ), baseando-se nos mesmos dados $y$, o vetor de parâmetros mais plausível será aquele com maior verossimilhança.

Para estimarmos o vetor de parâmetros verdadeiro $\theta_{0}$, devemos escolher o vetor de parâmetros que maximiza a função de verossimilhança no domínio de $\theta$. Assim, a estimativa de máxima verossimilhança $(E M V)$ de $\theta$ é o vetor $\widehat{\theta}$ que maximiza $L(\theta)$ em $\Theta$, isto é, $L(\widehat{\theta}) \geq L(\theta)$ para todo $\theta \in \Theta$.

Geralmente o processo de maximização é melhor conduzido trabalhando-se com o logaritmo natural de $L(\theta)$. Define-se o logaritmo natural da função de verossimilhança (também chamada de função suporte) por $l(\theta)=\log L(\theta)$. Assim, no caso de modelos normais não lineares,

$$
l(\theta)=-\frac{1}{2 \sigma^{2}} \sum_{i=1}^{n}\left[y_{i}-g_{i}(\nu)\right]^{2}-n \log \sigma .
$$

Como a função logaritmo é monótona crescente, maximizar $L(\theta)$ e $l(\theta)$ em $\Theta$ são processos equivalentes. Então a $E M V \hat{\theta}$ é definida de modo que, para todo $\theta \in \Theta$, $l(\widehat{\theta}) \geq l(\theta)$.

Como $l(\theta)$ é contínua e diferenciável em $\Theta=\mathbb{R}^{p+2}$, então a derivada de primeira 
ordem de $l(\theta)$ em relação a $\theta, l_{\theta}(\theta)=\partial l(\theta) / \partial \theta$, chamada de função (ou vetor) escore, é

$$
l_{\theta}(\theta)=\left[\begin{array}{c}
l_{\psi}(\theta) \\
l_{\beta}(\theta) \\
l_{\sigma}(\theta)
\end{array}\right]=\left[\begin{array}{c}
\frac{\partial l(\theta)}{\partial \psi} \\
\frac{\partial l(\theta)}{\partial \beta} \\
\frac{\partial l(\theta)}{\partial \sigma}
\end{array}\right]
$$

em que o operador $\partial / \partial \theta$ é interpretado como um vetor coluna e, portanto, $l_{\theta}(\theta)$ é um vetor $(p+2) \times 1$. Considere $l_{\lambda}(\theta)$ como sendo a derivada de primeira ordem do vetor $\left(\beta^{\top}, \sigma\right)^{\top}$, ou seja,

$$
l_{\lambda}(\theta)=\left[\begin{array}{l}
l_{\beta}(\theta) \\
l_{\sigma}(\theta)
\end{array}\right] .
$$

Neste caso, o vetor escore para o modelo normal não linear é composto por

$$
\begin{aligned}
& l_{\psi}(\theta)=\frac{1}{\sigma^{2}} \sum_{i=1}^{n}\left[y_{i}-g_{i}(\nu)\right] \frac{\partial g_{i}(\nu)}{\partial \psi} \\
& l_{\beta}(\theta)=\frac{1}{\sigma^{2}} \sum_{i=1}^{n}\left[y_{i}-g_{i}(\nu)\right] \frac{\partial g_{i}(\nu)}{\partial \beta}
\end{aligned}
$$

e

$$
l_{\sigma}(\theta)=\frac{1}{\sigma^{3}} \sum_{i=1}^{n}\left[y_{i}-g_{i}(\nu)\right]^{2}-\frac{n}{\sigma}
$$

A $E M V$ de $\theta$ é obtida da solução do sistema de equações simultâneas $l_{\theta}(\theta)=0$, que geralmente não têm solução analítica explícita. No final dessa seção são apresentados três métodos numéricos para a obtenção da $E M V$.

A matriz de derivadas de primeira ordem da função escore em relação a $\theta$, com sinal negativo, é denominada matriz de informação observada $(j(\theta))$ e é dada por

$$
j(\theta)=-l_{\theta \theta}(\theta)=-\left[\begin{array}{ccc}
l_{\psi \psi}(\theta) & l_{\psi \beta^{\top}}(\theta) & l_{\psi \sigma}(\theta) \\
l_{\beta \psi}(\theta) & l_{\beta \beta^{\top}}(\theta) & l_{\beta \sigma}(\theta) \\
l_{\sigma \psi}(\theta) & l_{\sigma \beta^{\top}}(\theta) & l_{\sigma \sigma}(\theta)
\end{array}\right]=-\left[\begin{array}{ccc}
\frac{\partial^{2} l(\theta)}{\partial \psi \partial \psi} & \frac{\partial^{2} l(\theta)}{\partial \psi \partial \beta^{\top}} & \frac{\partial^{2} l(\theta)}{\partial \psi \partial \sigma} \\
\frac{\partial^{2} l(\theta)}{\partial \beta \partial \psi} & \frac{\partial^{2} l(\theta)}{\partial \beta \partial \beta^{\top}} & \frac{\partial^{2} l(\theta)}{\partial \beta \partial \sigma} \\
\frac{\partial^{2} l(\theta)}{\partial \sigma \partial \psi} & \frac{\partial^{2} l(\theta)}{\partial \sigma \partial \beta^{\top}} & \frac{\partial^{2} l(\theta)}{\partial \sigma \partial \sigma}
\end{array}\right]
$$


Definimos $j_{\psi \psi}(\theta)=-l_{\psi \psi}(\theta)$ e

$$
j_{\lambda \lambda}(\theta)=-\left[\begin{array}{ll}
l_{\beta \beta^{\mathrm{T}}}(\theta) & l_{\beta \sigma}(\theta) \\
l_{\sigma \beta} \mathrm{\top}(\theta) & l_{\sigma \sigma}(\theta)
\end{array}\right] .
$$

Para o modelo normal não linear os elementos da matriz em (2.8) podem ser escritos como

$$
\begin{gathered}
-l_{\psi \psi}(\theta)=\frac{1}{\sigma^{2}}\left\{\sum_{i=1}^{n}\left(\frac{\partial g_{i}(\nu)}{\partial \psi}\right)^{2}-\sum_{i=1}^{n}\left[y_{i}-g_{i}(\nu)\right] \frac{\partial^{2} g_{i}(\nu)}{\partial \psi^{2}}\right\} \\
-l_{\psi \beta^{\top}}(\theta)=-l_{\beta \psi}(\theta)^{\top}=\frac{1}{\sigma^{2}}\left\{\sum_{i=1}^{n} \frac{\partial g_{i}(\nu)}{\partial \psi} \frac{\partial g_{i}(\nu)}{\partial \beta^{\top}}-\sum_{i=1}^{n}\left[y_{i}-g_{i}(\nu)\right] \frac{\partial^{2} g_{i}(\nu)}{\partial \psi \partial \beta^{\top}}\right\} \\
-l_{\psi \sigma}(\theta)=-l_{\sigma \psi}(\theta)=\frac{2}{\sigma^{3}} \sum_{i=1}^{n}\left[y_{i}-g_{i}(\nu)\right] \frac{\partial g_{i}(\nu)}{\partial \psi} \\
-l_{\beta \beta^{\top}}(\theta)=\frac{1}{\sigma^{2}}\left\{\sum_{i=1}^{n} \frac{\partial g_{i}(\nu)}{\partial \beta} \frac{\partial g_{i}(\nu)}{\partial \beta^{\top}}-\sum_{i=1}^{n}\left[y_{i}-g_{i}(\nu)\right] \frac{\partial^{2} g_{i}(\nu)}{\partial \beta \partial \beta^{\top}}\right\} \\
-l_{\beta \sigma}(\theta)=-l_{\sigma \beta} \top(\theta)^{\top}=\frac{2}{\sigma^{3}} \sum_{i=1}^{n}\left[y_{i}-g_{i}(\nu)\right] \frac{\partial g_{i}(\nu)}{\partial \beta}
\end{gathered}
$$

e

$$
-l_{\sigma \sigma}(\theta)=\frac{3}{\sigma^{4}} \sum_{i=1}^{n}\left[y_{i}-g_{i}(\nu)\right]^{2}-\frac{n}{\sigma^{2}} .
$$

Seja $i\left(\theta, \theta_{0}\right)=-E_{\theta_{0}}\left[l_{\theta}(\theta) l_{\theta}\left(\theta_{0}\right)^{\top}\right]$, em que $\theta_{0}=\left(\psi_{0}, \beta_{0}^{\top}, \sigma_{0}\right)^{\top}$ e $l_{\theta}(\theta)$ é a função escore. Assim,

$$
\begin{aligned}
& i\left(\theta, \theta_{0}\right)=-E_{\theta_{0}}\left\{\left[\begin{array}{l}
l_{\psi}(\theta) \\
l_{\beta}(\theta) \\
l_{\sigma}(\theta)
\end{array}\right]\left[\begin{array}{lll}
l_{\psi}\left(\theta_{0}\right) & l_{\beta^{\top}}\left(\theta_{0}\right) & l_{\sigma}\left(\theta_{0}\right)
\end{array}\right]\right\} \\
& =\left[\begin{array}{ccc}
i_{\psi \psi}\left(\theta, \theta_{0}\right) & i_{\psi \beta^{\top}}\left(\theta, \theta_{0}\right) & i_{\psi \sigma}\left(\theta, \theta_{0}\right) \\
i_{\beta \psi}\left(\theta, \theta_{0}\right) & i_{\beta \beta^{\top}}\left(\theta, \theta_{0}\right) & i_{\beta \sigma}\left(\theta, \theta_{0}\right) \\
i_{\sigma \psi}\left(\theta, \theta_{0}\right) & i_{\sigma \beta^{\top}}\left(\theta, \theta_{0}\right) & i_{\sigma \sigma}\left(\theta, \theta_{0}\right)
\end{array}\right] \text {. }
\end{aligned}
$$


A matriz $i(\theta)=i(\theta, \theta)$ é chamada de matriz de informação (ou matriz de informação esperada) de Fisher de $\theta$ contida nos dados y e tem dimensão $(p+2) \times(p+2)$. Para o modelo de regressão normal não linear,

$$
\begin{aligned}
i_{\psi \psi}\left(\theta, \theta_{0}\right) & =E_{\theta_{0}}\left\{\frac{1}{\sigma^{2} \sigma_{0}^{2}}\left[\sum_{j=1}^{n}\left[y_{i}-g_{i}(\nu)\right] \frac{\partial g_{i}(\nu)}{\partial \psi}\right]\left[\sum_{j=1}^{n}\left[y_{j}-g_{j}\left(\nu_{0}\right)\right] \frac{\partial g_{j}\left(\nu_{0}\right)}{\partial \psi}\right]\right\} \\
& =\frac{1}{\sigma^{2} \sigma_{0}^{2}} E_{\theta_{0}}\left\{\sum_{i=1}^{n} \sum_{j=1}^{n}\left[y_{i}-g_{i}(\nu)\right]\left[y_{j}-g_{j}\left(\nu_{0}\right)\right] \frac{\partial g_{i}(\nu)}{\partial \psi} \frac{\partial g_{j}\left(\nu_{0}\right)}{\partial \psi}\right\} .
\end{aligned}
$$

Mas,

$$
\begin{gathered}
\sum_{i=1}^{n} \sum_{j=1}^{n}\left[y_{i}-g_{i}(\nu)\right]\left[y_{j}-g_{j}\left(\nu_{0}\right)\right] \frac{\partial g_{i}(\nu)}{\partial \psi} \frac{\partial g_{j}\left(\nu_{0}\right)}{\partial \psi}= \\
\quad \sum_{i=1}^{n}\left[y_{i}-g_{i}(\nu)\right]\left[y_{i}-g_{i}\left(\nu_{0}\right)\right] \frac{\partial g_{i}(\nu)}{\partial \psi} \frac{\partial g_{i}\left(\nu_{0}\right)}{\partial \psi} \\
+\sum_{i \neq j}\left[y_{i}-g_{i}(\nu)\right]\left[y_{j}-g_{j}\left(\nu_{0}\right)\right] \frac{\partial g_{i}(\nu)}{\partial \psi} \frac{\partial g_{j}\left(\nu_{0}\right)}{\partial \psi}
\end{gathered}
$$

Aplicando a esperança em ambos os lados da igualdade, temos

$$
\begin{gathered}
\sum_{i=1}^{n} \frac{\partial g_{i}(\nu)}{\partial \psi} \frac{\partial g_{i}\left(\nu_{0}\right)}{\partial \psi} \mathrm{E}_{\theta_{0}}\left\{\left[y_{i}-g_{i}(\nu)\right]\left[y_{i}-g_{i}\left(\nu_{0}\right)\right]\right\} \\
+\sum_{i \neq j} \frac{\partial g_{i}(\nu)}{\partial \psi} \frac{\partial g_{j}\left(\nu_{0}\right)}{\partial \psi} \mathrm{E}_{\theta_{0}}\left\{\left[y_{i}-g_{i}(\nu)\right]\left[y_{j}-g_{j}\left(\nu_{0}\right)\right]\right\}= \\
\sum_{i=1}^{n} \frac{\partial g_{i}(\nu)}{\partial \psi} \frac{\partial g_{i}\left(\nu_{0}\right)}{\partial \psi} \sigma_{0}^{2},
\end{gathered}
$$

pois,

$$
\sum_{i \neq j} \frac{\partial g_{i}(\nu)}{\partial \psi} \frac{\partial g_{j}\left(\nu_{0}\right)}{\partial \psi} \mathrm{E}_{\theta_{0}}\left\{\left[y_{i}-g_{i}(\nu)\right]\left[y_{j}-g_{j}\left(\nu_{0}\right)\right]\right\}=0
$$


Assim,

$$
i_{\psi \psi}\left(\theta, \theta_{0}\right)=\frac{1}{\sigma^{2}} \sum_{i=1}^{n} \frac{\partial g_{i}(\nu)}{\partial \psi} \frac{\partial g_{i}\left(\nu_{0}\right)}{\partial \psi}
$$

Agora,

$$
\begin{aligned}
i_{\psi \beta^{\top}}\left(\theta, \theta_{0}\right) & =E_{\theta_{0}}\left\{\frac{1}{\sigma^{2} \sigma_{0}^{2}}\left[\sum_{j=1}^{n}\left[y_{i}-g_{i}(\nu)\right] \frac{\partial g_{i}(\nu)}{\partial \psi}\right]\left[\sum_{j=1}^{n}\left[y_{j}-g_{j}\left(\nu_{0}\right)\right] \frac{\partial g_{j}\left(\nu_{0}\right)}{\partial \beta^{\top}}\right]\right\} \\
& =\frac{1}{\sigma^{2} \sigma_{0}^{2}} E_{\theta_{0}}\left\{\sum_{i=1}^{n} \sum_{j=1}^{n}\left[y_{i}-g_{i}(\nu)\right]\left[y_{j}-g_{j}\left(\nu_{0}\right)\right] \frac{\partial g_{i}(\nu)}{\partial \psi} \frac{\partial g_{j}\left(\nu_{0}\right)}{\partial \beta^{\top}}\right\} \\
& =\frac{1}{\sigma^{2}} \sum_{i=1}^{n} \frac{\partial g_{i}(\nu)}{\partial \psi} \frac{\partial g_{i}\left(\nu_{0}\right)}{\partial \beta^{\top}}
\end{aligned}
$$

As demais quantidades necessárias para obtermos $i\left(\theta, \theta_{0}\right)$ são obtidas de forma análoga. Finalmente chegamos a

$$
i\left(\theta, \theta_{0}\right)=\frac{1}{\sigma^{2}}\left[\begin{array}{ccc}
z(\nu)^{\top} z\left(\nu_{0}\right) & z(\nu)^{\top} d\left(\nu_{0}\right) & 0 \\
d(\nu)^{\top} z\left(\nu_{0}\right) & d(\nu)^{\top} d\left(\nu_{0}\right) & 0 \\
(2 / \sigma) \delta\left(\nu, \nu_{0}\right)^{\top} z\left(\nu_{0}\right) & (2 / \sigma) \delta\left(\nu, \nu_{0}\right)^{\top} d\left(\nu_{0}\right) & n /\left(2 \sigma \sigma_{0}\right)
\end{array}\right]
$$

em que $z(\nu)$ é um vetor $(n \times 1)$ com o $i$-ésimo elemento dado por $\partial g_{i}(\nu) / \partial \psi, d(\nu)$ é uma matriz $(n \times p)$ com o $(i, j)$-ésimo elemento igual a $\partial g_{i}(\nu) / \partial \beta_{j}, \delta\left(\nu, \nu_{0}\right)$ é um vetor $(n \times 1)$ com o $i$-ésimo elemento dado por $g_{i}\left(\nu_{0}\right)-g_{i}(\nu)$. Nessa dissertação, 0 indica um vetor ou matriz de zeros de dimensão adequada. Em particular, em (2.9), 0 indica um vetor $p \times 1$ de zeros. Note que a matriz de informação de Fisher, $i(\theta)=i(\theta, \theta)$, é obtida fazendo $\theta_{0}=\theta$ e se reduz a

$$
i(\theta)=\frac{1}{\sigma^{2}}\left[\begin{array}{ccc}
z(\nu)^{\top} z(\nu) & z(\nu)^{\top} d(\nu) & 0 \\
d(\nu)^{\top} z(\nu) & d(\nu)^{\top} d(\nu) & 0 \\
0 & 0^{\top} & n / 2 \sigma^{2}
\end{array}\right]
$$

Em grandes amostras, sob condições de regularidade usuais,

$$
\widehat{\theta} \sim N\left(\theta, i(\theta)^{-1}\right)
$$


aproximadamente. Como a matriz de informação de Fisher é bloco diagonal, os parâmetros $\nu$ e $\sigma^{2}$ são assintoticamente independentes.

A matriz $i(\theta)$ pode ainda ser escrita como

$$
i(\theta)=\frac{1}{\sigma^{2}}\left[\begin{array}{cc}
F^{\top} F & 0 \\
0^{\top} & n / 2 \sigma^{2}
\end{array}\right],
$$

em que $F=\partial g(\nu) / \partial \nu^{\top}=\left[\begin{array}{ll}z(\nu) & d(\nu)\end{array}\right]$ de dimensão $n \times(p+1)$. Aqui, 0 é um vetor de dimensão $(p+1) \times 1$. A inversa de $i(\theta)$ é dada por

$$
i(\theta)^{-1}=\sigma^{2}\left[\begin{array}{cc}
\left(F^{\top} F\right)^{-1} & 0 \\
0^{\top} & 2 \sigma^{2} / n
\end{array}\right]
$$

No modelo de regressão normal linear a $E M V$ de $\theta$ poderá ser obtida resolvendo o sistema de equações simultâneas $l_{\theta}(\theta)=0$ para $\theta=\left(\psi, \beta^{\top}, \sigma\right)^{\top}$, ou seja, obtendo $\widehat{\theta}$ tal que $l_{\theta}(\widehat{\theta})=0$. Como mencionado anteriormente, no modelo de regressão normal não linear, em geral, não é possível obter uma forma analítica fechada para $\widehat{\theta}$. Neste caso, a obtenção da $E M V$ de $\theta$ é feita por algum método iterativo.

\section{Métodos Numéricos}

A seguir, serão descritos os métodos de Newton-Raphson, escore de Fisher (Cordeiro, 1992) e BFGS (Nocedal e Wright, 1999).

Expandindo $l_{\theta}(\widehat{\theta})$ (função escore em $\widehat{\theta}$ ) em série multivariada de Taylor até primeira ordem ao redor de um ponto qualquer $\theta$ pertencente a uma vizinhança de $\widehat{\theta}$, obtemos, aproximadamente,

$$
l_{\theta}(\widehat{\theta}) \doteq l_{\theta}(\theta)-j(\theta)(\widehat{\theta}-\theta)
$$

Como $l_{\theta}(\widehat{\theta})=0$ segue a relação aproximada

$$
\widehat{\theta}-\theta \doteq j(\theta)^{-1} l_{\theta}(\theta)
$$


entre a $E M V$, a função escore e a informação observada avaliadas no ponto $\theta$ próximo de $\widehat{\theta}$. O método iterativo de Newton-Raphson utilizado para o cálculo da $E M V$ consiste em utilizar a equação acima de forma iterativa. Obtemos uma nova estimativa $\theta^{(m+1)}$ a partir da anterior $\theta^{(m)}$ via

$$
\theta^{(m+1)}=\theta^{(m)}+j\left(\theta^{(m)}\right)^{-1} l_{\theta}\left(\theta^{(m)}\right), \quad m=0,1, \ldots
$$

em que quantidades avaliadas na $m$-ésima iteração do procedimento iterativo são indicadas pelo superescrito $(m)$. O processo é então repetido a partir de $\theta^{(m+1)}$ até a distância entre $\theta^{(m+1)}$ e $\theta^{(m)}$ se tornar desprezível ou menor que uma quantidade pequena especificada.

A expressão $\widehat{\theta}-\theta \doteq j(\theta)^{-1} l_{\theta}(\theta)$ tem uma forma alternativa equivalente assintoticamente pois, pela lei dos grandes números, $j(\theta)$ deve convergir para $i(\theta)$ quando $n \longrightarrow \infty$. Assim, substituindo a informação observada pela esperada, obtemos a aproximação

$$
\widehat{\theta}-\theta=i(\theta)^{-1} l_{\theta}(\theta)
$$

Esse procedimento é denominado método escore de Fisher e consiste na solução iterativa das equações

$$
\theta^{(m+1)}=\theta^{(m)}+i\left(\theta^{(m)}\right)^{-1} l_{\theta}\left(\theta^{(m)}\right), \quad m=0,1, \ldots
$$

Seja $S(\nu)=\sum_{i=1}^{n}\left[y_{i}-g_{i}(\nu)\right]^{2}$ (uma parcela de $l(\theta)$ dado em (2.6)). Fixado $\sigma^{2}$, maximizar $l(\theta)=l(\nu, \sigma)$ e minimizar $S(\nu)$, em relação a $\nu$, são processos equivalentes. A $E M V$ de $\nu$ independe de $\sigma^{2}$. Logo, as estimativas de máxima verossimilhança e de mínimos quadrados de $\nu$ coincidem. Fixado $\nu, \partial l(\theta) / \partial \sigma^{2}=0$ implica a solução $\widehat{\sigma}^{2}=$ $S(\nu) / n$, que representa um máximo, pois a derivada de segunda ordem é negativa. Como a $E M V$ de $\nu$ não depende do valor de $\sigma^{2}$, ela poderá ser calculada pelo método 
escore de Fisher, trabalhando apenas com a função escore e a informação de Fisher relativas a $\nu$. Da forma bloco diagonal da matriz de informação de Fisher e de sua inversa verifica-se que o método escore de Fisher obtém a $E M V \widehat{\nu}$ de $\nu$ através das equações

$$
\nu^{(m+1)}=\nu^{(m)}+\left\{\left(F^{\top} F\right)^{-1} F^{\top}(y-\mu)\right\}^{(m)}, \quad m=0,1, \ldots
$$

e a $E M V$ de $\sigma^{2}$ é $\widehat{\sigma}^{2}=S(\widehat{\nu}) / n$.

Além desses dois métodos iterativos apresentados, há ainda o método BFGS que foi desenvolvido por Broyden, Fletcher, Goldfarb e Shanno (ver Nocedal e Wright, 1999, Capítulo 8). Este método utiliza o mesmo princípio do método de NewtonRaphson, diferenciando-se pelo fato de utilizar uma seqüência de matrizes simétricas e positivas definidas $B^{(m)}$ no lugar da matriz $j\left(\theta^{(m)}\right)^{-1}$. Comumente, toma-se como matriz inicial, $B^{(0)}$, a matriz identidade de mesma ordem. A forma recursiva para obter as demais matrizes é dada por

$$
B^{(m+1)}=B^{(m)}-\frac{B^{(m)} s^{(m)}\left(s^{(m)}\right)^{\top} B^{(m)}}{\left(s^{(m)}\right)^{\top} B^{(m)} s^{(m)}}+\frac{c^{(m)}\left(c^{(m)}\right)^{\top}}{\left(c^{(m)}\right)^{\top} s^{(m)}} \quad m=0,1, \ldots
$$

em que $s^{(m)}=\theta^{(m+1)}-\theta^{(m)}$ e $c^{(m)}=j\left(\theta^{(m+1)}\right)-j\left(\theta^{(m)}\right)$. De forma análoga aos métodos anteriores, o máximo é obtido pela recorrência

$$
\theta^{(m+1)}=\theta^{(m)}-\left(j\left(\theta^{(m)}\right)\right)^{\top} B^{(m)}\left(\alpha^{(m)}\right)^{\top}, \quad m=0,1, \ldots
$$

em que $\alpha^{(m)}$ é um vetor de dimensão $1 \times(p+2)$ de escalares determinados por algum procedimento de busca linear a partir de $\theta^{(m)}$ na direção $-\left(j\left(\theta^{(m)}\right)\right)^{\top} B^{(m)}$ de forma que $f\left(y ; \theta^{(m)}\right)$ cresça nessa direção.

Este método está implementado na linguagem de programação ox através da função MaxBFGS. Maiores detalhes sobre este método podem ser encontrados em Nocedal e Wright (1999, Capítulo 8) e Doornik (2001, Seção 10.2). 


\subsection{Estatística da Razão de Verossimilhanças Si- nalizada $(R)$}

Severini (2000) apresenta a estatística da razão de verossimilhanças sinalizada para testes de hipóteses sobre $\psi$ definida por

$$
R \equiv R(\psi)=\operatorname{sinal}(\widehat{\psi}-\psi) \sqrt{2\left[l(\widehat{\theta})-l\left(\hat{\theta}_{\psi}\right)\right]}
$$

em que $\widehat{\theta}=\left(\widehat{\psi}, \widehat{\lambda}^{\top}\right)^{\top}, \widehat{\theta}_{\psi}=\left(\psi, \hat{\lambda}_{\psi}^{\top}\right)^{\top}, \widehat{\psi}$ e $\widehat{\lambda}$ são, respectivamente, os estimadores de máxima verossimilhança de $\psi$ e $\lambda$ e $\widehat{\lambda}_{\psi}$ é o estimador de máxima verossimilhança do vetor de parâmetros de perturbação $(\lambda)$ quando $\psi$ é fixado, ou seja, o valor de $\widehat{\lambda}_{\psi}$ é obtido maximizando o logaritmo da verossimilhança $(l(\theta))$ com respeito a $\lambda$ supondo $\psi$ constante.

Portanto, a estatística $R$ é dada por

$$
\begin{aligned}
R & =\sin a l(\widehat{\psi}-\psi)\left[-\frac{1}{\widehat{\sigma}^{2}} \sum_{i=1}^{n}\left[y_{i}-g_{i}(\widehat{\nu})\right]^{2}\right. \\
& \left.+\frac{1}{\widehat{\sigma}_{\psi}^{2}} \sum_{i=1}^{n}\left[y_{i}-g_{i}\left(\widehat{\nu}_{\psi}\right)\right]^{2}+2 n \log \left(\frac{\widehat{\sigma}_{\psi}}{\widehat{\sigma}}\right)\right]^{\frac{1}{2}} .
\end{aligned}
$$

Esta tem distribuição normal padrão com um erro de ordem $O\left(n^{-\frac{1}{2}}\right)$. Considerando as hipóteses estatísticas dadas no Capítulo $1, \psi$ deve ser substituído por $\psi_{0}$. 
Capítulo 3

\section{Ajustes para a Estatística da Razão de Verossimilhanças Sinalizada}

\subsection{Notação}

Para a obtenção das estatísticas da razão de verossimilhanças sinalizada modificadas citadas no Capítulo 1 é necessário introduzir a notação que se segue.

Vimos que $\theta=\left(\psi, \beta^{\top}, \sigma\right)^{\top}$ é um vetor de dimensão $(p+2) \times 1, \lambda=\left(\beta^{\top}, \sigma\right)^{\top}$ é um vetor de dimensão $(p+1) \times 1$, denominado parâmetro de perturbação, $\nu=\left(\psi, \beta^{\top}\right)^{\top}$ tem dimensão $(p+1) \times 1$, $\psi$ é o parâmetro (escalar) de interesse.

Seja $l(\theta ; \widehat{\theta}, a)$ o logaritmo natural da função de verossimilhança $(l(\theta))$ como função de $\theta, \widehat{\theta}$ (estimador de máxima verossimilhança de $\theta$ ) e, se necessário, uma estatística ancilar $a$, de tal forma que $(\widehat{\theta}, a)$ seja uma estatística suficiente para $\theta$. Seja $l_{; \widehat{\psi}}(\theta)=$ $\partial l(\theta ; \widehat{\theta}, a) / \partial \widehat{\psi}$ de dimensão $1 \times 1$. Assim, $l_{; \widehat{\psi}}(\widehat{\theta})=\partial l(\theta ; \widehat{\theta}, a) /\left.\partial \widehat{\psi}\right|_{\theta=\widehat{\theta}}$ e $l_{; \widehat{\psi}}\left(\widehat{\theta}_{\psi}\right)=$ $\partial l(\theta ; \widehat{\theta}, a) /\left.\partial \widehat{\psi}\right|_{\theta=\widehat{\theta}_{\psi}}$, ambos de dimensão $1 \times 1$. De forma análoga, definem-se $l_{; \widehat{\lambda}^{\top}}(\theta)$, $l_{; \hat{\lambda}^{\top}}(\widehat{\theta})$ e $l_{; \bar{\lambda}^{\top}}\left(\widehat{\theta}_{\psi}\right)$ 
As quantidades $l_{\lambda ; \widehat{\psi}}(\widehat{\theta})$ e $l_{\lambda ; \widehat{\psi}}\left(\widehat{\theta}_{\psi}\right)$ igualam-se a $l_{\lambda ; \widehat{\psi}}(\theta)=\partial^{2} l(\theta ; \widehat{\theta}, a) / \partial \widehat{\psi} \partial \lambda$ avaliados em $\theta=\widehat{\theta}$ e $\theta=\widehat{\theta}_{\psi}$, respectivamente. Note que

$$
l_{\lambda ; \widehat{\psi}}\left(\widehat{\theta}_{\psi}\right)=\left[\begin{array}{c}
l_{\beta ; \widehat{\psi}}\left(\widehat{\theta}_{\psi}\right) \\
l_{\sigma ; \widehat{\psi}}\left(\widehat{\theta}_{\psi}\right)
\end{array}\right]=\left[\begin{array}{c}
\frac{\partial}{\partial \widehat{\psi}}\left(\frac{\partial}{\partial \beta} l(\theta ; \hat{\theta}, a)\right) \\
\frac{\partial}{\partial \widehat{\psi}}\left(\frac{\partial}{\partial \sigma} l(\theta ; \widehat{\theta}, a)\right)
\end{array}\right]_{\theta=\widehat{\theta}_{\psi}}
$$

que possui dimensão $(p+1) \times 1$. Da mesma maneira temos

$$
l_{\lambda ; \hat{\lambda}^{\top}}\left(\widehat{\theta}_{\psi}\right)=\left[\begin{array}{cc}
l_{\beta ; \widehat{\beta}^{\top}}\left(\widehat{\theta}_{\psi}\right) & l_{\beta ; \sigma ;}\left(\widehat{\theta}_{\psi}\right) \\
l_{\sigma ; \widehat{\beta}^{\top}}\left(\widehat{\theta}_{\psi}\right) & l_{\sigma ; ; \sigma}\left(\widehat{\theta}_{\psi}\right)
\end{array}\right]
$$

que possui dimensão $(p+1) \times(p+1)$.

No Capítulo 2 foram definidas em (2.8) a matriz de informação observada $(j(\theta))$ e, a partir de (2.9), a matriz de informação de Fisher $(i(\theta))$. Ao longo deste capítulo utilizaremos $j(\widehat{\theta})$, a matriz de informação observada avaliada em $\theta=\widehat{\theta}$, a submatriz $j_{\lambda \lambda}\left(\widehat{\theta}_{\psi}\right)$, que também é obtida de $j(\theta)$, esta avaliada em $\theta=\widehat{\theta}_{\psi}$. Dessa forma, para obter $j_{\lambda \lambda}\left(\hat{\theta}_{\psi}\right)$, basta avaliar as quantidades $l_{\beta \beta^{\Upsilon}}(\theta), l_{\beta \sigma}(\theta)$ e $l_{\sigma \sigma}(\theta)$ dadas em (2.8) em $\theta=\widehat{\theta}_{\psi}$, ou seja,

$$
j_{\lambda \lambda}\left(\widehat{\theta}_{\psi}\right)=\left.j_{\lambda \lambda}(\theta)\right|_{\theta=\widehat{\theta}_{\psi}}=\left[\begin{array}{ll}
-l_{\beta \beta} \mathrm{T}(\theta) & -l_{\beta \sigma}(\theta) \\
-l_{\sigma \beta} \mathrm{T}(\theta) & -l_{\sigma \sigma}(\theta)
\end{array}\right]_{\theta=\widehat{\theta}_{\psi}} .
$$

As quantidades $i_{\psi \psi}(\widehat{\theta})$ e $i_{\psi \psi}\left(\widehat{\theta}_{\psi}\right)$, correspondem ao elemento $(1,1)$ da matriz de informação de Fisher avaliado em $\theta=\widehat{\theta}$ e $\theta=\widehat{\theta}_{\psi}$, respectivamente.

\subsection{Estatística da Razão de Verossimilhanças Si- nalizada Modificada $\left(R^{*}\right)$}

Nesta seção, apresentaremos a estatística da razão de verossimilhanças sinalizada modificada $\left(R^{*}\right)$ proposta por Barndorff-Nielsen $(1986,1991)$, que possui assintoti- 
camente uma distribuição normal padrão com um erro de ordem $O\left(n^{-\frac{3}{2}}\right)$, isto é,

$$
\mathrm{P}\left(R^{*} \leq t\right)=\Phi(t)\left[1+O\left(n^{-3 / 2}\right)\right]
$$

para valores fixados de $t$ (Severini, 2000, p.249). Aqui, $\Phi(\cdot)$ é a função de distribuição acumulada da normal padrão. Note que a estatística $R$ tem essa mesma distribuição porém, com um erro de ordem $O\left(n^{-\frac{1}{2}}\right)$. Espera-se, portanto, que em amostras pequenas e de tamanho moderado a distribuição de $R^{*}$ tenha uma melhor aproximação pela distribuição $\mathrm{N}(0,1)$ do que a distribuição de $R$. A estatística $R^{*}$ é definida como

$$
R^{*}=R+\frac{1}{R} \log \left(\frac{U}{R}\right)
$$

em que

$$
U=\frac{\left|\begin{array}{c}
l_{; \widehat{\theta}}(\widehat{\theta})-l_{; \widehat{\theta}}\left(\widehat{\theta}_{\psi}\right) \\
l_{\lambda ; \widehat{\theta}}\left(\widehat{\theta}_{\psi}\right)
\end{array}\right|}{|j(\widehat{\theta})|^{\frac{1}{2}}\left|j_{\lambda \lambda}\left(\widehat{\theta}_{\psi}\right)\right|^{\frac{1}{2}}}
$$

Utilizando a fórmula para o determinante de matriz particionada, dada em Rao (1973), uma forma alternativa para obter a estatística $U$ é

$$
U=\frac{\left|l_{\lambda ; \hat{\lambda}}\left(\widehat{\theta}_{\psi}\right)\right|\left|l_{; \widehat{\psi}}(\widehat{\theta})-l_{; \widehat{\psi}}\left(\widehat{\theta}_{\psi}\right)-l_{\lambda ; \widehat{\psi}}\left(\widehat{\theta}_{\psi}\right)^{\top} l_{\lambda ; \hat{\lambda}}\left(\widehat{\theta}_{\psi}\right)^{-1}\left[l_{\hat{\lambda}, \hat{\theta}}(\widehat{\theta})^{\top}-l_{; \hat{\lambda}}\left(\widehat{\theta}_{\psi}\right)^{\top}\right]\right|}{|j(\widehat{\theta})|^{\frac{1}{2}}\left|j_{\lambda \lambda}\left(\widehat{\theta}_{\psi}\right)\right|^{\frac{1}{2}}}
$$

Segundo Severini (2000), para obter a estatística $U$ em (3.2) é necessário escrever o logaritmo da função de verossimilhança em função de $\theta=\left(\psi, \beta^{\top}, \sigma\right)^{\top}$, $\widehat{\theta}=\left(\widehat{\psi}, \widehat{\beta}^{\top}, \widehat{\sigma}\right)^{\top}$, a estimativa de máxima verossimilhança de $\theta$, e, se necessário, uma estatística ancilar a. Posteriormente, calcular a derivada com respeito ao espaço amostral. Isto só será possível se o modelo for linear, caso em que $\widehat{\theta}$ tem forma fechada. Sendo assim, mostraremos como é o cálculo de $R^{*}$ no modelo de regressão normal linear, com $g_{i}(\nu)$ dado em (2.3). Denotaremos por $X$ a matriz de planejamento 
relacionada ao vetor de parâmetros $\nu$, em que $X_{i}=\left(X_{i 1}, \ldots, X_{i s}\right)^{\top}$, com $i=1, \ldots, n$. A matriz referente às covariadas (conhecidas) relacionadas aos parâmetros $\beta$ 's será denotada por $x$, em que $x_{i}=\left(X_{i 2}, \ldots, X_{i s}\right)^{\top}$, com $i=1, \ldots, n, \sum_{i=1}^{n} x_{i}=0$ e $e$, o vetor coluna de uns de dimensão $n \times 1$. Logo, a matriz $X$ também pode ser denotada como $X=\left(e, x^{\top}\right)^{\top}$. Nesse caso, a função densidade de probabilidade de $Y_{i}$ dada em (2.2) tem a forma

$$
f\left(y_{i} ; \theta\right)=\frac{1}{\sqrt{2 \pi} \sigma} \exp \left\{-\frac{\left[y_{i}-X_{i}^{\top} \nu\right]^{2}}{2 \sigma^{2}}\right\}
$$

e a função de verossimilhança é

$$
L(\theta)=\frac{1}{(\sqrt{2 \pi} \sigma)^{n}} \exp \left\{-\frac{\sum_{i=1}^{n}\left[y_{i}-X_{i}^{\top} \nu\right]^{2}}{2 \sigma^{2}}\right\}
$$

lembrando que $\nu=\left(\psi, \beta^{\top}\right)^{\top}$. Note que

$$
\sum_{i=1}^{n}\left[y_{i}-X_{i}^{\top} \nu\right]^{2}=n \widehat{\sigma}^{2}+n(\widehat{\psi}-\psi)^{2}+(\widehat{\beta}-\beta)^{\top} x^{\top} x(\widehat{\beta}-\beta) .
$$

Logo, o logaritmo da função de verossimilhança com apenas as parcelas que dependem de $\theta$ e $\widehat{\theta}$ fica dado por

$$
l(\theta)=-n \log \sigma-\frac{1}{2 \sigma^{2}}\left[n \widehat{\sigma}^{2}+n(\widehat{\psi}-\psi)^{2}+(\widehat{\beta}-\beta)^{\top} x^{\top} x(\widehat{\beta}-\beta)\right] .
$$

Para obtermos a estatística da razão de verossimilhanças sinalizada $(R)$ é necessário avaliar o logaritmo da função de verossimilhança $(l(\theta))$ em $\theta=\widehat{\theta}$ e $\theta=\widehat{\theta}_{\psi}$. A quantidade $\sum_{i=1}^{n}\left[y_{i}-X_{i}^{\top} \nu\right]^{2}$ avaliada, respectivamente, em $\theta=\widehat{\theta}$ e $\theta=\widehat{\theta}_{\psi}$ é dada por

$$
\sum_{i=1}^{n}\left[y_{i}-X_{i}^{\top} \widehat{\nu}\right]^{2}=n \widehat{\sigma}^{2}
$$

$\mathrm{e}$

$$
\sum_{i=1}^{n}\left[y_{i}-X_{i}^{\top} \widehat{\nu}_{\psi}\right]^{2}=n \widehat{\sigma}^{2}+n(\widehat{\psi}-\psi)^{2}+\left(\widehat{\beta}-\widehat{\beta}_{\psi}\right)^{\top} x^{\top} x\left(\widehat{\beta}-\widehat{\beta}_{\psi}\right)
$$

Agora, como $\widehat{\sigma}_{\psi}^{2}=\sum_{i=1}^{n}\left[y_{i}-X_{i}^{\top} \widehat{\nu}_{\psi}\right]^{2} / n$,

$$
n \widehat{\sigma}_{\psi}^{2}=n \widehat{\sigma}^{2}+n(\widehat{\psi}-\psi)^{2}+\left(\widehat{\beta}-\widehat{\beta}_{\psi}\right)^{\top} x^{\top} x\left(\widehat{\beta}-\widehat{\beta}_{\psi}\right) .
$$


Consequentemente,

$$
l(\widehat{\theta})=-n \log \widehat{\sigma}-\frac{n}{2}
$$

e

$$
l\left(\widehat{\theta}_{\psi}\right)=-n \log \widehat{\sigma}_{\psi}-\frac{n}{2} .
$$

Portanto, a estatística $R$, definida em (2.10), se reduz nesse caso particular a

$$
R=\operatorname{sinal}(\widehat{\psi}-\psi)\left[2 n\left(\log \frac{\widehat{\sigma}_{\psi}}{\widehat{\sigma}}\right)\right]^{\frac{1}{2}}
$$

Agora obteremos algumas quantidades que serão necessárias para a obtenção do numerador de $U$ :

$$
\begin{aligned}
& l_{; \widehat{\psi}}(\widehat{\theta})-l_{; \widehat{\psi}}\left(\widehat{\theta}_{\psi}\right)=\frac{n(\widehat{\psi}-\psi)}{\widehat{\sigma}_{\psi}^{2}} \\
& l_{; \hat{\lambda}^{\top}}(\widehat{\theta})-l_{; \widehat{\lambda}^{\top}}\left(\widehat{\theta}_{\psi}\right)=\left[\frac{\left(\widehat{\beta}-\widehat{\beta}_{\psi}\right)^{\top} x^{\top} x}{\widehat{\sigma}_{\psi}^{2}}-n\left(\frac{1}{\widehat{\sigma}}-\frac{\widehat{\sigma}}{\widehat{\sigma}_{\psi}^{2}}\right)\right] \\
& l_{\lambda ; \hat{\psi}}(\widehat{\theta})=\left[\begin{array}{c}
0 \\
\frac{2 n(\hat{\psi}-\psi)}{\widehat{\sigma}_{\psi}^{3}}
\end{array}\right] \quad \text { e } \quad l_{\lambda ; \hat{\lambda}^{\top}}\left(\widehat{\theta}_{\psi}\right)=\left[\begin{array}{cc}
\frac{x^{\top} x}{\widehat{\sigma}_{\psi}^{2}} & 0 \\
\frac{2\left(\widehat{\beta}-\widehat{\beta}_{\psi}\right)^{\top} x^{\top} x}{\widehat{\sigma}_{\psi}^{3}} & \frac{2 n \widehat{\sigma}}{\widehat{\sigma}_{\psi}^{3}}
\end{array}\right] \text {. }
\end{aligned}
$$

Finalmente, o numerador de $U$ definido em (3.2) é

$$
\frac{2 n^{2}(\widehat{\psi}-\psi)\left|x^{\top} x\right|}{\widehat{\sigma} \widehat{\sigma}_{\psi}^{2 p+3}}
$$

Para a obtenção do denominador de $U$ temos

$$
|j(\widehat{\theta})|=\frac{2 n^{2}\left|x^{\top} x\right|}{\widehat{\sigma}^{2 p+4}}
$$

e

$$
\left|j_{\lambda \lambda}\left(\widehat{\theta}_{\psi}\right)\right|=\frac{2 n\left|x^{\top} x\right|}{\widehat{\sigma}_{\psi}^{2 p+2}}
$$


Das expressões para o numerador e denominador de $U$, chegamos finalmente a

$$
U=\frac{\widehat{\sigma}^{p+1}}{\widehat{\sigma}_{\psi}^{p+2}} \sqrt{n}(\widehat{\psi}-\psi) .
$$

Logo, basta substituir em (3.1) as expressões (3.4) e (3.7) para obter $R^{*}$.

\subsection{Aproximações para $R^{*}$}

\subsubsection{Introdução}

Embora o uso de $R^{*}$ acarrete uma melhoria no teste da razão de verossimilhanças sinalizado, existe a desvantagem de que a obtenção de $R^{*}$ é frequentemente difícil. A dificuldade está no cálculo de $U$ que requer a determinação de derivadas em relação ao espaço amostral (ver (3.1) e (3.2)). Como discutido na Seção 3.2, o cálculo exato dessas derivadas geralmente requer que possamos escrever o logaritmo da função de verossimilhança em função de $\theta, \hat{\theta}$ e, se necessário, uma estatística ancilar a (Severini, 2000). Esta dificuldade inviabiliza a obtenção de $R^{*}$ no caso de modelos não lineares.

Nessa seção, apresentamos aproximações para $R^{*}$ através de diversos métodos e em ordem cronológica. Na Seção 3.3.2, a aproximação é baseada em parâmetros ortogonais $\left(R_{0}^{*}\right)$. Já os métodos descritos nas Seções 3.3 .3 a 3.3 .5 correspondem a aproximações baseadas em covariâncias $\left(\bar{R}^{*}\right)$, covariâncias empíricas $\left(\widehat{R}^{*}\right)$ e numa estatística aproximadamente ancilar $\left(\widetilde{R}^{*}\right)$, respectivamente. Uma descrição desses métodos pode ser encontrada em Severini (2000, Capítulo 7). 


\subsubsection{Estatística da Razão de Verossimilhanças Sinalizada Modificada baseada em Parâmetros Ortogonais $\left(R_{0}^{*}\right)$}

Suponha que $\psi$, o parâmetro de interesse primário, é ortogonal a $\lambda$, o parâmetro de perturbação, isto é, admita que $\mathrm{E}\left\{(\partial l(\theta) / \partial \psi)\left[\partial l(\theta) / \partial \lambda^{\top}\right]\right\}=0$, o que significa que a matriz de informação de Fisher é bloco diagonal. Com essa suposição, DiCiccio e Martin (1993) propuseram uma aproximação para $R^{*}$ que chamaremos de estatística da razão de verossimilhanças sinalizada baseada em parâmetros ortogonais $\left(R_{0}^{*}\right)$. Note que a matriz de informação de Fisher obtida para o caso de modelos normais não lineares, dada logo após a expressão $(2.9)$, mostra que $\nu=\left(\psi, \beta^{\top}\right)^{\top}$ é ortogonal a $\sigma^{2}$, mas que $\psi$ e $\beta$ não são ortogonais. Isso impossibilita o uso desta estatística, pois para utilizá-la é necessário que $\psi$ seja ortogonal a $\lambda=\left(\beta^{\top}, \sigma\right)^{\top}$. Como no caso de modelos normais lineares, com $\sum_{i=1}^{n} x_{i}=0, \psi$ é ortogonal a $\lambda$, obteremos esta estatística para esse caso particular. A estatística proposta por DiCiccio e Martin (1993) é dada por

$$
R_{0}^{*}=R+\frac{1}{R} \log \left(\frac{U_{0}}{R}\right)
$$

em que

$$
U_{0}=l_{\psi}\left(\widehat{\theta}_{\psi}\right) \frac{\left|j_{\lambda \lambda}\left(\widehat{\theta}_{\psi}\right)\right|^{\frac{1}{2}}\left(i_{\psi \psi}(\widehat{\theta})\right)^{\frac{1}{2}}}{|j(\widehat{\theta})|^{\frac{1}{2}}\left(i_{\psi \psi}\left(\widehat{\theta}_{\psi}\right)\right)^{\frac{1}{2}}}
$$

e

$$
R_{0}^{*}=R^{*}+O_{p}\left(n^{-1}\right)
$$

para $\psi$ da forma $\widehat{\psi}+O\left(n^{-1 / 2}\right)$; ver Severini $(2000$, p.263).

Vimos na Seção 3.2 que o logaritmo da função de verossimilhança para o caso linear é dado por (3.3) e que o determinante de $j(\widehat{\theta})$ e $j_{\lambda \lambda}\left(\widehat{\theta}_{\psi}\right)$ são dados em (3.5) e 
(3.6), respectivamente. As demais quantidades necessárias para obtermos $U_{0}$ são:

$$
\begin{gathered}
l_{\psi}\left(\widehat{\theta}_{\psi}\right)=\left.\frac{\partial l(\theta)}{\partial \psi}\right|_{\theta=\widehat{\theta}_{\psi}}=\frac{n(\widehat{\psi}-\psi)}{\widehat{\sigma}_{\psi}^{2}}, \\
i_{\psi \psi}(\widehat{\theta})=E\left[-l_{\psi \psi}(\theta)\right]_{\theta=\widehat{\theta}}=E\left[-\frac{\partial^{2} l(\theta)}{\partial \psi^{2}}\right]_{\theta=\widehat{\theta}}=\frac{n}{\widehat{\sigma}^{2}}
\end{gathered}
$$

e

$$
i_{\psi \psi}\left(\widehat{\theta}_{\psi}\right)=\frac{n}{\widehat{\sigma}_{\psi}^{2}}
$$

Assim,

$$
U_{0}=\frac{\widehat{\sigma}^{p+1}}{\widehat{\sigma}_{\psi}^{p+2}} \sqrt{n}(\widehat{\psi}-\psi)
$$

que coincide com a expressão de $U$ em (3.7).

Portanto, $R_{0}^{*}$ é dada por (3.8) com $R$ dado em (3.4) e $U_{0}$ dado em (3.10). Note que como as estatísticas $R_{0}^{*}$ e $R^{*}$ coincidem, a aproximação de DiCiccio e Martin (1993), nesse caso, é exata.

\subsubsection{Estatística da Razão de Verossimilhanças Sinalizada Modificada baseada em Covariâncias $\left(\bar{R}^{*}\right)$}

A estatística $\bar{R}^{*}$, baseada em covariâncias, foi proposta por Skovgaard (1996) como uma aproximação para $R^{*}$. Ela é dada por

$$
\bar{R}^{*}=R+\frac{1}{R} \log \left(\frac{\bar{U}}{R}\right)
$$

em que

$$
\bar{U}=\frac{\left|\begin{array}{c}
\bar{l}_{; \widehat{\theta}}(\widehat{\theta})-\bar{l}_{; \widehat{\theta}}\left(\widehat{\theta}_{\psi}\right) \\
\bar{l}_{\lambda ; \hat{\theta}}\left(\widehat{\theta}_{\psi}\right)
\end{array}\right|}{|j(\widehat{\theta})|^{\frac{1}{2}}\left|j_{\lambda \lambda}\left(\widehat{\theta}_{\psi}\right)\right|^{\frac{1}{2}}}
$$


com

$$
\begin{gathered}
\bar{l}_{; \hat{\theta}}(\widehat{\theta})-\bar{l}_{; \widehat{\theta}}\left(\widehat{\theta}_{\psi}\right)=\left[Q(\widehat{\theta}, \widehat{\theta})-Q\left(\widehat{\theta}_{\psi}, \widehat{\theta}\right)\right][i(\widehat{\theta})]^{-1} j(\widehat{\theta}) \\
\bar{l}_{\theta ; \hat{\theta}}\left(\widehat{\theta}_{\psi}\right)=i\left(\widehat{\theta}_{\psi}, \widehat{\theta}\right)[i(\widehat{\theta})]^{-1} j(\widehat{\theta}) \\
Q\left(\theta, \theta_{0}\right)=\mathrm{E}_{\theta_{0}}\left[l(\theta) l_{\theta}\left(\theta_{0}\right)^{\top}\right] \\
i\left(\theta, \theta_{0}\right)=\mathrm{E}_{\theta_{0}}\left[l_{\theta}(\theta) l_{\theta}\left(\theta_{0}\right)^{\top}\right]
\end{gathered}
$$

e $i(\widehat{\theta})=i(\hat{\theta}, \widehat{\theta})$. Essa é uma aproximação para $R^{*}$ pois, de Severini (2000, p.269), temos que $\bar{R}^{*}=R^{*}+O_{p}\left(n^{-1}\right)$, para $\psi$ da forma $\widehat{\psi}+O\left(n^{-1 / 2}\right)$.

Vimos que para a obtenção da estatística $R^{*}$ é necessário obter separadamente as quantidades $l_{; \hat{\theta}}(\widehat{\theta}), l_{; \hat{\theta}}\left(\hat{\theta}_{\psi}\right)$ e $l_{\lambda ; \hat{\theta}}\left(\widehat{\theta}_{\psi}\right)$ que são derivadas em relação ao espaço amostral e não são possíveis de serem obtidas em modelos normais não lineares. A proposta do Skovgaard (1996) é usar aproximações para as quantidades $l_{; \widehat{\theta}}(\widehat{\theta})-l_{; \widehat{\theta}}\left(\widehat{\theta}_{\psi}\right)$ e $l_{\lambda ; \hat{\theta}}\left(\widehat{\theta}_{\psi}\right)$ que não requerem o cálculo destas derivadas. Essas quantidades são aproximadas por covariâncias e, por isso, a estatística $\bar{R}^{*}$ é chamada dessa forma, ou seja,

$$
\begin{aligned}
\operatorname{Cov}_{\theta_{0}}\left(l_{\theta}(\theta), l_{\theta}\left(\theta_{0}\right)\right) & =\mathrm{E}_{\theta_{0}}\left(l_{\theta}(\theta) l_{\theta}\left(\theta_{0}\right)\right)-\mathrm{E}_{\theta_{0}}\left(l_{\theta}(\theta)\right) \mathrm{E}_{\theta_{0}}\left(l_{\theta}\left(\theta_{0}\right)\right) \\
& =\mathrm{E}_{\theta_{0}}\left(l_{\theta}(\theta) l_{\theta}\left(\theta_{0}\right)\right)=i\left(\theta, \theta_{0}\right)
\end{aligned}
$$

em que a covariância é denotada por Cov sendo igual a $i\left(\theta, \theta_{0}\right)$ definida no Capítulo 2, em (2.9) e é utilizada nas quantidades (3.13) e (3.14).

Para o modelo de regressão normal, a quantidade $Q\left(\theta, \theta_{0}\right)$ utilizada em (3.13) é dada por

$$
Q\left(\theta, \theta_{0}\right)=-\frac{1}{\sigma^{2}}\left[\delta\left(\nu, \nu_{0}\right)^{\top} z\left(\nu_{0}\right) \quad \delta\left(\nu, \nu_{0}\right)^{\top} d\left(\nu_{0}\right) \quad n \sigma_{0}\right]
$$

em que $z(\cdot), d(\cdot)$ e $\delta(\cdot)$ foram definidos em (2.9). Consequentemente

$$
Q(\widehat{\theta}, \widehat{\theta})-Q\left(\widehat{\theta}_{\psi}, \widehat{\theta}\right)=\left[\begin{array}{lll}
\frac{\delta\left(\widehat{\nu}_{\psi}, \widehat{\nu}\right)^{\top} z(\widehat{\nu})}{\widehat{\sigma}_{\psi}^{2}} & \frac{\delta\left(\widehat{\nu}_{\psi}, \widehat{\nu}\right)^{\top} d(\widehat{\nu})}{\widehat{\sigma}_{\psi}^{2}} & \left(\frac{n \widehat{\sigma}}{\widehat{\sigma}_{\psi}^{2}}-\frac{n}{\bar{\sigma}}\right)
\end{array}\right],
$$


com $\delta\left(\widehat{\nu}_{\psi}, \widehat{\nu}\right)=g(\widehat{\nu})-g\left(\widehat{\nu}_{\psi}\right)$ de dimensão $n \times 1$. A matriz de informação de Fisher, definida após a expressão (2.9), avaliada em $\theta=\widehat{\theta}$, é dada, nesse caso, por

$$
i(\widehat{\theta})=\frac{1}{\widehat{\sigma}^{2}}\left[\begin{array}{ccc}
z(\widehat{\nu})^{\top} z(\widehat{\nu}) & z(\widehat{\nu})^{\top} d(\widehat{\nu}) & 0 \\
d(\widehat{\nu})^{\top} z(\widehat{\nu}) & d(\widehat{\nu})^{\top} d(\widehat{\nu}) & 0 \\
0 & 0 & \frac{n}{2 \widehat{\sigma}^{2}}
\end{array}\right]
$$

e $j(\widehat{\theta})$ contida na expressão (3.13) é a informação observada dada em (2.8) avaliada em $\theta=\widehat{\theta}$. Portanto, substituindo a quantidade $Q(\widehat{\theta}, \widehat{\theta})-Q\left(\widehat{\theta}_{\psi}, \widehat{\theta}\right)$, a inversa de $i(\widehat{\theta})$ e a matriz $j(\widehat{\theta})$ na expressão dada em (3.13) resulta uma matriz de dimensão $1 \times(p+2)$.

A quantidade

$$
i\left(\widehat{\theta}_{\psi}, \widehat{\theta}\right)=\frac{1}{\widehat{\sigma}_{\psi}^{2}}\left[\begin{array}{ccc}
z\left(\widehat{\nu}_{\psi}\right)^{\top} z(\widehat{\nu}) & z\left(\widehat{\nu}_{\psi}\right)^{\top} d(\widehat{\nu}) & 0 \\
d\left(\widehat{\nu}_{\psi}\right)^{\top} z(\widehat{\nu}) & d\left(\widehat{\nu}_{\psi}\right)^{\top} d(\widehat{\nu}) & 0 \\
\left(2 / \widehat{\sigma}_{\psi}\right) \delta\left(\widehat{\nu}_{\psi}, \widehat{\nu}\right)^{\top} z(\widehat{\nu}) & \left(2 / \widehat{\sigma}_{\psi}\right) \delta\left(\widehat{\nu}_{\psi}, \widehat{\nu}\right)^{\top} d(\widehat{\nu}) & n /\left(2 \widehat{\sigma} \widehat{\sigma}_{\psi}\right)
\end{array}\right]
$$

obtida de (2.9) $\operatorname{com} \theta=\widehat{\theta}_{\psi}$ e $\theta_{0}=\widehat{\theta}$ é utilizada para obter (3.14). Assim, a multiplicação de $i\left(\widehat{\theta}_{\psi}, \widehat{\theta}\right)$, a inversa de $i(\widehat{\theta})$ e a matriz $j(\widehat{\theta})$ resulta na matriz (3.14), de dimensão $(p+2) \times(p+2)$.

O numerador de $\bar{U}$ (ver (3.12)) envolve as quantidades (3.13) e $\bar{l}_{\lambda ; \hat{\theta}}\left(\widehat{\theta}_{\psi}\right)$. Para obter $\bar{l}_{\lambda ; \hat{\theta}}\left(\widehat{\theta}_{\psi}\right)$ é necessário descartar a primeira linha da matriz obtida em (3.14), obtendo assim uma matriz de dimensão $(p+1) \times(p+2)$. Logo, a matriz resultante (no numerador de $\vec{U}$ ) terá a primeira linha com o resultado da expressão (3.13) de dimensão $1 \times(p+2)$ e as demais linhas com a matriz de dimensão $(p+1) \times(p+2)$, resultado de $\bar{l}_{\lambda ; \hat{\theta}}\left(\widehat{\theta}_{\psi}\right)$.

Finalmente, $\bar{R}^{*}$ será como em (3.11) com $R$ dado em (2.11) e $\bar{U}$ dado em (3.12) obtido a partir das expressões acima. 


\subsubsection{Estatística da Razão de Verossimilhanças Sinalizada Modificada baseada em Covariâncias Empíricas $\left(\widehat{R}^{*}\right)$}

Vimos na seção anterior que a estatística $\bar{R}^{*}$ foi calculada com base em aproximações para determinadas quantidades, em que essas aproximações eram baseadas em covariâncias. Podemos substituir as covariâncias utilizadas anteriormente por covariâncias empíricas sem mudar a ordem de aproximação. A estatística $\widehat{R}^{*}$ baseada em covariâncias empíricas foi proposta por Severini (1999). Ela é dada por

$$
\widehat{R}^{*}=R+\frac{1}{R} \log \left(\frac{\widehat{U}}{R}\right),
$$

em que

$$
\widehat{U}=\frac{\left|\begin{array}{c}
\hat{l}_{; \widehat{\theta}}(\widehat{\theta})-\widehat{l}_{; \widehat{\theta}}\left(\widehat{\theta}_{\psi}\right) \\
\widehat{l}_{\lambda ; \widehat{\theta}}\left(\widehat{\theta}_{\psi}\right)
\end{array}\right|}{|j(\widehat{\theta})|^{\frac{1}{2}}\left|j_{\lambda \lambda}\left(\widehat{\theta}_{\psi}\right)\right|^{\frac{1}{2}}}
$$

com

$$
\begin{gathered}
\left.\widehat{l}_{; ;}(\widehat{\theta})-\widehat{l}_{;}\left(\widehat{\theta}_{\psi}\right)=\left[\widehat{Q}(\widehat{\theta}, \widehat{\theta})-\widehat{Q}\left(\widehat{\theta}_{\psi}, \widehat{\theta}\right)\right] \hat{i}(\widehat{\theta})\right]^{-1} j(\widehat{\theta}), \\
\widehat{l}_{\theta ; \hat{\theta}}\left(\widehat{\theta}_{\psi}\right)=\widehat{i}\left(\widehat{\theta}_{\psi} ; \widehat{\theta}\right)[\widehat{i}(\widehat{\theta})]^{-1} j(\widehat{\theta}), \\
\widehat{Q}\left(\theta ; \theta_{0}\right)=\sum_{i} l^{(i)}(\theta) l_{\theta}^{(i)}\left(\theta_{0}\right)^{\top} \\
\widehat{i}\left(\theta ; \theta_{0}\right)=\sum_{i} l_{\theta}^{(i)}(\theta) l_{\theta}^{(i)}\left(\theta_{0}\right)^{\top}
\end{gathered}
$$

e

$$
\widehat{i}(\widehat{\theta})=\widehat{i}(\widehat{\theta} ; \widehat{\theta}) .
$$


A estatística baseada em covariâncias empíricas $\left(\widehat{R}^{*}\right)$ é uma aproximação para $R^{*}$ pois, de Severini $\left(2000\right.$, p.273), temos $\widehat{R}^{*}=R^{*}+O_{p}\left(n^{-1}\right)$, para $\psi$ da forma $\widehat{\psi}+O\left(n^{-1 / 2}\right)$.

Analogamente à estatística anterior, as quantidades em (3.17) e (3.18) são aproximações para $l_{; \hat{\theta}}(\widehat{\theta})-l_{; \hat{\theta}}\left(\widehat{\theta}_{\psi}\right)$ e $l_{\lambda ; \hat{\theta}}\left(\widehat{\theta}_{\psi}\right)$ que também não requerem o cálculo das derivadas em relação ao espaço amostral. Aqui, as quantidades dadas em $\widehat{U}$ são aproximadas por covariâncias empíricas e, por isso, a estatística $\widehat{R}^{*}$ é chamada dessa forma.

O termo $l^{(i)}(\theta)$ é o logaritmo da função de verossimilhança baseado apenas na $i$-ésima observação. Nos modelos normais não lineares, o logaritmo da função de verossimilhança baseado apenas na $i$-ésima observação é o logaritmo natural da densidade de $Y_{i}$ dada em (2.2). Logo,

$$
l^{(i)}(\theta)=-\log \sigma-\frac{\left[y_{i}-g_{i}(\nu)\right]^{2}}{2 \sigma^{2}} .
$$

A quantidade $l_{\theta}^{(i)}(\theta)$ é a derivada de $l^{(i)}(\theta)$ em relação a $\theta$. Assim,

em que

$$
l_{\theta}^{(i)}(\theta)=\left[\begin{array}{c}
l_{\psi}^{(i)}(\theta) \\
l_{\beta}^{(i)}(\theta) \\
l_{\sigma}^{(i)}(\theta)
\end{array}\right]=\left[\begin{array}{c}
\frac{\partial l^{(i)}(\theta)}{\partial \psi} \\
\frac{\partial l^{(i)}(\theta)}{\partial \beta} \\
\frac{\partial l^{(i)}(\theta)}{\partial \sigma}
\end{array}\right]
$$

$$
\begin{aligned}
& l_{\psi}^{(i)}(\theta)=\frac{1}{\sigma^{2}}\left[y_{i}-g_{i}(\nu)\right] \frac{\partial g_{i}(\nu)}{\partial \psi} \\
& l_{\beta}^{(i)}(\theta)=\frac{1}{\sigma^{2}}\left[y_{i}-g_{i}(\nu)\right] \frac{\partial g_{i}(\nu)}{\partial \beta}
\end{aligned}
$$

e

$$
l_{\sigma}^{(i)}(\theta)=\frac{1}{\sigma^{3}}\left[y_{i}-g_{i}(\nu)\right]^{2}-\frac{1}{\sigma}
$$

Logo,

$$
\widehat{Q}(\widehat{\theta}, \widehat{\theta})-\widehat{Q}\left(\widehat{\theta}_{\psi}, \widehat{\theta}\right)=\sum_{i} l^{(i)}(\widehat{\theta}) l_{\theta}^{(i)}(\widehat{\theta})^{\top}-\sum_{i} l^{(i)}\left(\widehat{\theta}_{\psi}\right) l_{\theta}^{(i)}(\widehat{\theta})^{\top}
$$


A quantidade $\widehat{i}(\widehat{\theta})$ fica dada por

$$
\hat{i}(\widehat{\theta})=\widehat{i}(\widehat{\theta}, \widehat{\theta})=\sum_{i} l_{\theta}^{(i)}(\widehat{\theta}) l_{\theta}^{(i)}(\widehat{\theta})^{\top}
$$

Assim, substituindo $\widehat{Q}(\widehat{\theta}, \widehat{\theta})-\widehat{Q}\left(\widehat{\theta}_{\psi}, \widehat{\theta}\right)$, a inversa de $\widehat{i}(\widehat{\theta})$ e a matriz $j(\widehat{\theta})$, que é a informação observada dada em (2.8) avaliada em $\theta=\widehat{\theta}$, obtemos a matriz (3.17) de dimensão $1 \times(p+2)$. Finalmente, a quantidade

$$
\widehat{i}\left(\widehat{\theta}_{\psi} ; \widehat{\theta}\right)=\sum_{i} l_{\theta}^{(i)}\left(\widehat{\theta}_{\psi}\right) l_{\theta}^{(i)}(\widehat{\theta})^{\top}
$$

é utilizada para a obtenção da matriz em (3.18).

Portanto, basta multiplicar a matriz $\widehat{i}\left(\widehat{\theta}_{\psi}, \widehat{\theta}\right)$, a inversa de $\widehat{i}(\widehat{\theta})$ e a matriz $j(\widehat{\theta})$ para chegar à matriz dada em (3.18), de dimensão $(p+2) \times(p+2)$.

O numerador de $\widehat{U}$ (ver (3.16)) contém as quantidades (3.17) e $\widehat{l}_{\lambda ; \hat{\theta}}\left(\widehat{\theta}_{\psi}\right)$. Para obtermos $\widehat{l}_{\lambda ; \widehat{\theta}}\left(\widehat{\theta}_{\psi}\right)$ é necessário descartar a primeira linha da matriz obtida em (3.18), assim teremos uma matriz de dimensão $(p+1) \times(p+2)$. Logo, a matriz resultante (no numerador de $\widehat{U}$ ) terá a primeira linha com o resultado da expressão (3.17) de dimensão $1 \times(p+2)$ e as demais linhas com a matriz $\widehat{l}_{\lambda ; \widehat{\theta}}\left(\widehat{\theta}_{\psi}\right)$ de dimensão $(p+1) \times$ $(p+2)$.

Portanto $\widehat{R}^{*}$ é dada como em (3.15), com $\widehat{U}$ dado em (3.16), obtido das expressões acima e $R$ dado em (2.11).

\subsubsection{Estatística da Razão de Verossimilhanças Sinalizada Modificada baseada numa Estatística Aproximadamente Ancilar $\left(\widetilde{R}^{*}\right)$}

A estatística $\widetilde{R}^{*}$, baseada numa estatística aproximadamente ancilar, é a mais recente aproximação para $R^{*}$ considerada nessa dissertação e foi proposta por Fraser, 
Reid e Wu (1999). Ela é dada por

$$
\widetilde{R}^{*}=R+\frac{1}{R} \log \left(\frac{\widetilde{U}}{R}\right),
$$

em que

$$
\widetilde{U}=\frac{\left|\begin{array}{c}
\tilde{l}_{; \hat{\theta}}(\widehat{\theta})-\tilde{l}_{; \widehat{\theta}}\left(\widehat{\theta}_{\psi}\right) \\
\tilde{l}_{\lambda ; \hat{\theta}}\left(\widehat{\theta}_{\psi}\right)
\end{array}\right|}{|j(\widehat{\theta})|^{\frac{1}{2}}\left|j_{\lambda \lambda}\left(\widehat{\theta}_{\psi}\right)\right|^{\frac{1}{2}}}
$$

com

$$
\begin{gathered}
\tilde{l}_{; \hat{\theta}}(\theta)=l_{; y}(\theta) \widehat{V}\left[l_{\theta ; y}(\widehat{\theta}) \widehat{V}\right]^{-1} j(\widehat{\theta}), \\
\widetilde{l}_{\theta ; \hat{\theta}}\left(\widehat{\theta}_{\psi}\right)=l_{\theta ; y}\left(\widehat{\theta}_{\psi}\right) \widehat{V}\left[l_{\theta ; y}(\widehat{\theta}) \widehat{V}\right]^{-1} j(\widehat{\theta})
\end{gathered}
$$

e

$$
\widehat{V}=\left[\begin{array}{c}
-\frac{\partial F\left(y_{1} ; \widehat{\theta}\right) / \partial \widehat{\theta}}{f\left(y_{1} ; \widehat{\theta}\right)} \\
\vdots \\
-\frac{\partial F\left(y_{n} ; \widehat{\theta}\right) / \partial \widehat{\theta}}{f\left(y_{n} ; \hat{\theta}\right)}
\end{array}\right] .
$$

Severini $\left(2000\right.$, p.216) mostra que $F\left(y_{i} ; \widehat{\theta}\right)$ é uma estatística aproximadamente ancilar, pois $\mathrm{P}_{\theta}\left[F\left(Y_{i} ; \widehat{\theta}\right) \leq t\right]=t+O\left(n^{-1}\right)$. Além disso, $\widetilde{R}^{*}$ é uma aproximação para $R^{*}$ pois, de Severini (2000), temos que $\widetilde{R}^{*}=R^{*}+O_{p}\left(n^{-3 / 2}\right)$, para $\psi$ da forma $\widehat{\psi}+O\left(n^{-1 / 2}\right)$. Sob a distribuição com parâmetro $\psi, \widetilde{R}^{*}$ é assintoticamente distribuída como uma normal padrão com erro $O\left(n^{-3 / 2}\right)$.

Considere $Y_{1}, Y_{2}, \ldots, Y_{n}$ variáveis aleatórias independentes, com distribuição normal com média $g_{i}(\nu)$, variância constante $\sigma^{2}$, função de distribuição acumulada $F\left(Y_{i} ; \theta\right)$ e função densidade $f\left(y_{i} ; \theta\right)$ (dada em $(2.2)$ ). Como as $Y_{i}^{\prime}$ 's possuem distribuição $\mathrm{N}\left(g_{i}(\nu), \sigma^{2}\right)$, então

$$
F\left(Y_{i} ; \theta\right)=\Phi\left[\frac{Y_{i}-g_{i}(\nu)}{\sigma}\right]
$$


em que $\Phi[\cdot]$ é a função de distribuição acumulada normal padrão. Assim,

$$
\begin{aligned}
\widehat{V} & =\left[\begin{array}{ccccc}
\frac{\partial g_{1}}{\partial \psi}(\widehat{\nu}) & \frac{\partial g_{1}}{\partial \beta_{1}}(\widehat{\nu}) & \cdots & \frac{\partial g_{1}}{\partial \beta_{p}}(\widehat{\nu}) & \frac{y_{1}-g_{1}(\widehat{\nu})}{\widehat{\sigma}} \\
\vdots & \vdots & \vdots & \vdots & \vdots \\
\frac{\partial g_{n}}{\partial \psi}(\widehat{\nu}) & \frac{\partial g_{n}}{\partial \beta_{1}}(\widehat{\nu}) & \ldots & \frac{\partial g_{n}}{\partial \beta_{p}}(\widehat{\nu}) & \frac{y_{n}-g_{n}(\widehat{\nu})}{\widehat{\sigma}}
\end{array}\right] \\
& =\left[\begin{array}{lll}
z(\widehat{\nu}) & d(\widehat{\nu}) & \gamma(\widehat{\nu})
\end{array}\right]
\end{aligned}
$$

em que $z(\cdot)$ e $d(\cdot)$ foram definidas logo após a equação $(2.9)$ e $\gamma(\cdot)$ é um vetor $(n \times 1)$ com o $i$-ésimo elemento dado por $\left[y_{i}-g_{i}(\nu)\right] / \sigma$.

Logo, resta calcular $l_{i y}(\theta)$ e $l_{\theta ; y}(\theta)$. O termo $l_{; y}(\theta)$ é a derivada de $l(\theta)$ em relação ao vetor $y=\left(y_{1}, y_{2}, \cdots, y_{n}\right)^{\top}$ e é dado por

$$
l_{; y}(\theta)=\left[\begin{array}{lll}
-\frac{1}{\sigma^{2}}\left(y_{1}-g_{1}(\nu)\right) & \cdots & -\frac{1}{\sigma^{2}}\left(y_{n}-g_{n}(\nu)\right)
\end{array}\right]^{\top}
$$

e assim, $l_{; y}(\widehat{\theta})$ e $l_{; y}\left(\widehat{\theta}_{\psi}\right)$ são obtidos avaliando a expressão acima em $\widehat{\theta}$ e $\widehat{\theta}_{\psi}$.

A quantidade $l_{\theta ; y}(\theta)$ é a derivada da função escore $\left(l_{\theta}(\theta)\right)$ em relação a $y$ e é dada por

$$
l_{\theta ; y}(\theta)=\frac{1}{\sigma^{2}}\left[\begin{array}{c}
z(\nu)^{\top} \\
d(\nu)^{\top} \\
2 \gamma(\nu)^{\top}
\end{array}\right]
$$

Consequentemente,

$$
l_{\theta ; y}(\widehat{\theta})=\frac{1}{\widehat{\sigma}^{2}}\left[\begin{array}{c}
z(\widehat{\nu})^{\top} \\
d(\widehat{\nu})^{\top} \\
2 \gamma(\widehat{\nu})^{\top}
\end{array}\right]
$$

e

$$
l_{\theta ; y}\left(\widehat{\theta}_{\psi}\right)=\frac{1}{\widehat{\sigma}_{\psi}^{2}}\left[\begin{array}{c}
z\left(\widehat{\nu}_{\psi}\right)^{\top} \\
d\left(\widehat{\nu}_{\psi}\right)^{\top} \\
2 \gamma\left(\widehat{\nu}_{\psi}\right)^{\top}
\end{array}\right]
$$


As quantidades $\widetilde{l}_{; \widehat{\theta}}(\widehat{\theta})$ e $\widetilde{l}_{;}\left(\widehat{\theta}_{\psi}\right)$ são obtidas avaliando (3.21) em $\widehat{\theta}$ e $\widehat{\theta}_{\psi}$, respectivamente. Assim, obtemos $\widetilde{l}_{; \hat{\theta}}(\widehat{\theta})-\widetilde{l}_{; \hat{\theta}}\left(\widehat{\theta}_{\psi}\right)$ de dimensão $1 \times(p+2)$.

O numerador de $\widetilde{U}$ (ver (3.20)) contém a quantidade (3.21) avaliada em $\widehat{\theta}$ e $\widehat{\theta}_{\psi}$ e $\widetilde{l}_{\lambda ; \widehat{\theta}}\left(\widehat{\theta}_{\psi}\right)$. Para obtermos $\widetilde{l}_{\lambda ; \hat{\theta}}\left(\widehat{\theta}_{\psi}\right)$ é necessário descartar a primeira linha da matriz obtida em (3.22) e assim teremos uma matriz de dimensão $(p+1) \times(p+2)$. Logo, a matriz resultante (no numerador de $\widetilde{U}$ ) terá a primeira linha igual a matriz $\widetilde{l}_{; \widehat{\theta}}(\widehat{\theta})-$ $\widetilde{l}_{;}\left(\widehat{\theta}_{\psi}\right)$ de dimensão $1 \times(p+2)$ e as demais linhas com a matriz $\widetilde{l}_{\lambda ; \hat{\theta}}\left(\widehat{\theta}_{\psi}\right)$ de dimensão $(p+1) \times(p+2)$.

Portanto, $\widetilde{R}^{*}$ é dado como em (3.19), com $\widetilde{U}$ dado em (3.20) e $R$ dado em (2.11). 
Capítulo 4

\section{Estudos de Simulação}

\subsection{Introdução}

Nesse capítulo avaliamos numericamente o comportamento da estatística da razão de verossimilhanças sinalizada e suas versões modificadas, apresentadas no capítulo anterior, em amostras finitas. O objetivo é comparar o teste baseado na estatística da razão de verossimilhanças sinalizada com os testes baseados nas versões modificadas em amostras finitas, sob diversas condições. Serão levados em conta a probabilidade de erro do tipo I, correspondente à rejeição de hipóteses nulas unilaterais definidas no Capítulo 1 e, o poder dos testes. Os testes que apresentam taxa de rejeição superior ao nível de significância nominal são chamados de liberais. Esses testes levam à rejeição da hipótese nula erroneamente com probabilidade mais alta que o desejado. O oposto disso são os testes conservativos. À primeira vista, pode parecer uma característica desejável o fato de um teste ser conservativo, uma vez que nessa situação o erro tipo I ocorre com baixa probabilidade. No entanto, em geral, testes conservativos apresentam um baixo poder. Assim, busca-se identificar testes que tenham tamanhos empíricos razoavelmente próximos (ou menores) ao nível de significância nominal e, ao mesmo tempo, tenham um alto poder. 


\subsection{Detalhes Metodológicos}

Os resultados numéricos apresentados a seguir referem-se aos testes baseados nas estatísticas dadas no Capítulo 3 aplicadas ao modelo de regressão normal linear

$$
Y_{i}=\psi+\beta_{1} x_{i 1}+\ldots+\beta_{p} x_{i p}+\sigma \varepsilon_{i}
$$

e nos modelos de regressão normais não lineares

$$
Y_{i}=\beta_{1}+\exp \left(\psi x_{i 1}\right)+\beta_{2} x_{i 2}+\ldots+\beta_{p} x_{i p}+\sigma \varepsilon_{i}
$$

e

$$
Y_{i}=\frac{\beta_{1} x_{i 1}}{\psi+x_{i 1}}+\beta_{2} x_{i 2}+\ldots+\beta_{p} x_{i p}+\sigma \varepsilon_{i}
$$

com $i=1,2, \ldots, n$, em que o parâmetro de interesse nos três modelos é $\psi$, ou seja, no modelo (4.1) o interesse recai no intercepto e nos modelos normais não lineares (4.2) e (4.3), no coeficiente da variável $x_{1}$. Mais precisamente, o interesse é testar $H_{0}: \psi \leq 0$ versus $H_{1}: \psi>0$.

Os estudos de simulação foram desenvolvidos utilizando-se a linguagem de programação matricial 0x (Doornik, 2001). Para as simulações em que as estatísticas foram obtidas baseando-se nos modelos (4.1) e (4.2) tomou-se o valor 1 para os parâmetros de perturbação $\left(\beta_{1}, \ldots, \beta_{p}, \sigma\right)$ e zero para o parâmetro de interesse $(\psi)$. Quando foi utilizado o modelo (4.3) tomou-se o mesmo valor para os parâmetros $\sigma(\sigma=1)$ e $\psi(\psi=0)$, mas, para o vetor $\beta$ de dimensão $p \times 1$, tomamos como valor inicial $(40, \ldots, 40)^{\top}$. No caso linear, a estimação dos parâmetros foi baseada no método de mínimos quadrados, enquanto que para o caso não linear, as maximizações não-lineares necessárias para o cálculo das estimativas de máxima verossimilhança foram feitas utilizando-se o algoritmo quasi-Newton BFGS, apresentado no Capítulo 2 e disponível em uma função pré-definida da linguagem $0 x$. No modelo linear, a 
primeira coluna da matriz de covariáveis correspondente ao parâmetro $\psi$ é de uns. As demais colunas foram obtidas gerando valores independentemente da distribuição $\mathcal{U}(0,1)$ e depois foi aplicada a tranformação $x-\bar{x}$, para que a soma de cada coluna relacionada ao vetor de parâmetros $\beta$ fosse 0 e assim obtermos $\psi$ e $\lambda=\left(\beta^{\top}, \sigma\right)^{\top}$ ortogonais. Logo, poderemos verificar o desempenho dos testes baseados nas seis estatísticas $\left(R, R^{*}, R_{0}^{*}, \bar{R}^{*}, \widehat{R}^{*}\right.$ e $\left.\widetilde{R}^{*}\right)$. Para os modelos não lineares a matriz de covariáveis foram obtidas a partir da distribuição $\mathcal{U}(0,1)$ sem nenhuma transformação e as estatísticas que devem ser obtidas, nesse caso, são $R, \vec{R}^{*}, \widehat{R}^{*}$ e $\widetilde{R}^{*}$.

Em uma primeira etapa, foram feitas simulações para a avaliação dos tamanhos empíricos dos testes. Buscou-se variar os elementos que podem influenciar o tamanho dos testes em amostras finitas. Tais elementos consistem no tamanho amostral $(n)$, número de parâmetros de perturbação $(p+1)$ e distribuição das variáveis independentes (covariáveis). Os testes foram realizados em 10000 amostras simuladas e baseados nos níveis nominais de $10 \%, 5 \%, 1 \%$ e $0,5 \%$. Foram obtidas as porcentagens dos valores das estatísticas que foram maiores que os quantis da distribuição normal padrão correspondentes aos níveis de significância adotados. A segunda etapa das simulações consistiu no estudo do poder dos testes sob algumas situações. Todas as entradas das tabelas apresentadas correspondem a porcentagens.

\subsection{Tamanho dos Testes}

Vamos considerar o caso do modelo normal linear (4.1). Para esta situação, podemos obter todas as seis estatísticas, mas como as estatísticas $R^{*}$ e $R_{0}^{*}$ são equivalentes, apresentaremos somente os resultados referentes à estatística $R^{*}$.

Primeiramente mostraremos os tamanhos dos testes para os tamanhos amostrais 
$n=10,20$ e 30 , fixando a dimensão do vetor $\beta$ em $p=4$ e as covariáveis geradas independentemente da distribuição $\mathcal{U}(0,1)$ e tal que a $\sum_{i=1}^{n} x_{i}=0$. Depois fixamos o tamanho amostral $(n=20)$, variamos a dimensão do vetor $\beta$ em $p=2,5 \mathrm{e}$ 8 e as covariáveis geradas independentemente da distribuição $\mathcal{U}(0,1)$ e tal que a $\sum_{i=1}^{n} x_{i}=0$. Finalmente, fixamos o tamanho amostral $(n=20)$, a dimensão de $\beta$ $(p=4)$ e geramos valores para as colunas $2, \ldots,(p+1)$ de $X$ das distribuições $t$ Student com 3 graus de liberdade e $t$-Student com 10 graus de liberdade, que possuem caudas pesadas e, qui-quadrado com 5 graus de liberdade, que é assimétrica.

A Tabela 4.1 contém os resultados dos tamanhos dos testes para as situações em que $n=10,20$ e $30, p=4$ e as covariáveis são geradas da distribuição $\mathcal{U}(0,1)$, ou seja, esta tabela apresenta as porcentagens em que $R, R^{*}, \bar{R}^{*}, \widehat{R}^{*}$ e $\widetilde{R}^{*}$ excederam os quantis da distribuição normal padrão obtidos sob os níveis nominais de $10 \%$, $5 \%, 1 \%$ e $0,5 \%$. Observando a Tabela 4.1, fica claro que os tamanhos empíricos baseados nas estatísticas ajustadas tendem a ser mais próximos dos correspondentes níveis nominais do que o tamanho empírico baseado na estatística $R$. Podemos notar também que o teste baseado na estatística $R$, para os tamanhos amostrais considerados, mostrou-se liberal no sentido de que as taxas de rejeição do teste baseado em $R$ estão sempre acima dos níveis nominais considerados. Como esperado, para tamanhos amostrais pequenos, como $n=10$, os tamanhos empíricos baseados em $R$, estão mais distantes dos níveis nominais considerados. Evidentemente, conforme cresce o tamanho amostral, os tamanhos empíricos vão se aproximando dos respectivos níveis nominais. Assim, as modificações para a estatística $R$ se tornam mais necessárias para tamanhos amostrais pequenos. Por exemplo, para $n=10$ e um nível nominal $\alpha=5 \%$, os tamanhos empíricos dos testes baseados nas estatísticas $R, R^{*}, \bar{R}^{*}, \widehat{R}^{*}$ e $\widetilde{R}^{*}$ são $13,2 \%, 8,2 \%, 5,5 \%, 8,1 \%$ e $6,1 \%$, respectivamente. Claramente é notado que a taxa de rejeição do teste baseado na estatística $R$ é muito mais distante de $5 \%$ 
que as taxas de rejeição dos testes baseados nas estatísticas ajustadas. Comparando os tamanhos empíricos dos testes baseados nas estatísticas ajustadas, o teste que apresenta uma taxa de rejeição observada mais próxima do nível nominal é o que utiliza a estatística $\bar{R}^{*}$. Em geral, destacou-se, com melhor desempenho nas situações apresentadas na Tabela 4.1, a estatística $\bar{R}^{*}$ proposta por Skovgaard (1996).

Tabela 4.1: Tamanhos empíricos dos testes no modelo normal linear (4.1), para $n=10,20$ e $30, p=4$ e covariáveis uniformes.

\begin{tabular}{ccccccc}
\hline \hline$n$ & Níveis Nominais & $R$ & $R^{*}$ & $\bar{R}^{*}$ & $\widehat{R}^{*}$ & $\widetilde{R}^{*}$ \\
\hline 10 & $10 \%$ & 18,8 & 14,6 & 10,9 & 12,9 & 11,4 \\
& $5 \%$ & 13,2 & 8,2 & 5,5 & 8,1 & 6,1 \\
& $1 \%$ & 5,6 & 2,2 & 1,0 & 3,5 & 1,4 \\
& $0,5 \%$ & 4,1 & 1,3 & 0,5 & 2,4 & 0,8 \\
\hline 20 & $10 \%$ & 13,4 & 10,4 & 10,5 & 9,3 & 10,0 \\
& $5 \%$ & 7,9 & 5,3 & 5,2 & 4,3 & 4,8 \\
& $1 \%$ & 2,2 & 1,3 & 1,1 & 0,9 & 1,2 \\
& $0,5 \%$ & 1,5 & 0,7 & 0,6 & 0,5 & 0,6 \\
\hline 30 & $10 \%$ & 12,3 & 10,3 & 10,5 & 9,9 & 10,1 \\
& $5 \%$ & 7,1 & 5,3 & 5,5 & 4,9 & 5,1 \\
& $1 \%$ & 1,8 & 1,1 & 1,1 & 0,9 & 1,0 \\
& $0,5 \%$ & 1,0 & 0,5 & 0,5 & 0,5 & 0,5 \\
\hline \hline
\end{tabular}

A Tabela 4.2 apresenta os resultados dos tamanhos empíricos quando o tamanho amostral é 20, as covariáveis são geradas da distribuição uniforme e a dimensão do vetor $\beta$ varia em $p=2,5$ e 8 . Observando a Tabela 4.2, os tamanhos empíricos do teste baseado na estatística $R$, mostraram-se acima dos níveis nominais considerados, ou seja, o teste baseado nesta estatística mostrou-se liberal. Conforme se diminui a dimensão do vetor $\beta$, os tamanhos empíricos vão se aproximando dos níveis nominais 
adotados. Para uma dimensão maior do vetor $\beta$, a aproximação para a distribuição normal padrão da estatística $R$ é deteriorada.

Tabela 4.2: Tamanhos empíricos dos testes no modelo normal linear (4.1), para $p=2,5$ e $8, n=20$ e covariáveis uniformes.

\begin{tabular}{ccccccc}
\hline \hline$p$ & Níveis Nominais & $R$ & \multicolumn{1}{c}{$R^{*}$} & $\bar{R}^{*}$ & $\widehat{R}^{*}$ & $\widetilde{R}^{*}$ \\
\hline 2 & $10 \%$ & 11,8 & 10,0 & 10,2 & 9,5 & 9,6 \\
& $5 \%$ & 6,4 & 4,8 & 5,1 & 4,5 & 4,7 \\
& $1 \%$ & 1,6 & 1,1 & 1,1 & 1,0 & 1,0 \\
& $0,5 \%$ & 1,0 & 0,7 & 0,7 & 0,6 & 0,7 \\
\hline 5 & $10 \%$ & 14,3 & 10,9 & 10,6 & 8,5 & 10,2 \\
& $5 \%$ & 8,5 & 5,3 & 5,0 & 3,9 & 4,7 \\
& $1 \%$ & 2,7 & 1,2 & 1,1 & 0,7 & 1,1 \\
& $0,5 \%$ & 1,6 & 0,6 & 0,6 & 0,3 & 0,6 \\
\hline 8 & $10 \%$ & 17,1 & 12,3 & 11,1 & 7,2 & 11,0 \\
& $5 \%$ & 11,4 & 6,5 & 5,5 & 3,2 & 5,6 \\
& $1 \%$ & 4,3 & 1,5 & 1,2 & 0,6 & 1,2 \\
& $0,5 \%$ & 2,9 & 1,0 & 0,7 & 0,3 & 0,8 \\
\hline \hline
\end{tabular}

Ainda da Tabela 4.2, podemos notar que os testes baseados nas estatísticas ajustadas se tornam mais necessários para os casos em que o número de parâmetros de perturbação $(p+1)$ é alto. Por exemplo, para $p=8$ e um nível nominal $\alpha=5 \%$, os tamanhos empíricos dos testes que usam $R, R^{*}, \bar{R}^{*}, \widehat{R}^{*}$ e $\widetilde{R}^{*}$ são $11,4 \%, 6,5 \%, 5,5 \%$, $3,2 \%$ e $5,6 \%$, respectivamente. Podemos notar facilmente o quão distante está a taxa de rejeição do teste baseado em $R$ de $5 \%$, enquanto que as taxas de rejeição dos testes baseados nas estatísticas ajustadas estão bem mais próximas deste nível nominal. $O$ teste baseado em $\widehat{R}^{*}$, nas situações apresentadas nessa tabela, mostrou-se conservativo, no sentido de que as taxas de rejeição de $\widehat{R}^{*}$ estão abaixo dos níveis nominais 
correspondentes, ou seja, o erro tipo I está ocorrendo com probabilidade menor do que o esperado. Em geral, podemos observar que, nas situações apresentadas na Tabela 4.2 , as estatísticas que apresentaram melhor desempenho foram $\bar{R}^{*}, \widehat{R}^{*}$ e $\widetilde{R}^{*}$, pois os testes que utilizaram essas estatísticas forneceram tamanhos empíricos mais próximos ou inferiores aos níveis nominais considerados.

Fixando o tamanho amostral $n=20$ e a dimensão do vetor $\beta$ em $p=4$, temos na Tabela 4.3 os tamanhos dos testes para covariáveis geradas das distribuições $t$ Student com 3 graus de liberdade, $t$-Student com 10 graus de liberdade e $\chi^{2}$ com 5 graus de liberdade.

Tabela 4.3: Tamanhos empíricos dos testes no modelo normal linear (4.1), para $p=4, n=20$ e covariáveis com distribuição $t_{3}, t_{10}$ e $\chi_{5}^{2}$.

\begin{tabular}{ccrrrrr}
\hline \hline Dist. & Niveis Nominais & \multicolumn{1}{c}{$R$} & \multicolumn{1}{c}{$R^{*}$} & $\bar{R}^{*}$ & $\widehat{R}^{*}$ & $\widetilde{R}^{*}$ \\
\hline$t_{3}$ & $10 \%$ & 13,4 & 10,6 & 10,5 & 9,5 & 10,1 \\
& $5 \%$ & 7,9 & 5,4 & 5,3 & 4,5 & 5,0 \\
& $1 \%$ & 2,3 & 1,3 & 1,2 & 0,9 & 1,2 \\
& $0,5 \%$ & 1,5 & 0,7 & 0,7 & 0,5 & 0,7 \\
\hline$t_{10}$ & $10 \%$ & 13,2 & 10,4 & 10,5 & 8,7 & 10,0 \\
& $5 \%$ & 7,6 & 5,2 & 5,1 & 4,3 & 4,8 \\
& $1 \%$ & 2,2 & 1,3 & 1,1 & 1,0 & 1,0 \\
& $0,5 \%$ & 1,3 & 0,6 & 0,6 & 0,5 & 0,5 \\
\hline$\chi_{5}^{2}$ & $10 \%$ & 13,3 & 10,4 & 10,4 & 8,9 & 9,9 \\
& $5 \%$ & 7,7 & 5,3 & 5,1 & 4,4 & 4,8 \\
& $1 \%$ & 2,1 & 1,2 & 1,1 & 0,9 & 1,1 \\
& $0,5 \%$ & 1,4 & 0,8 & 0,7 & 0,5 & 0,7 \\
\hline \hline
\end{tabular}


Podemos observar nessa tabela que o teste baseado na estatística $R$ apresentou taxas de rejeição acima dos níveis nominais correspondentes. Observamos ainda que, independentemente das distribuições consideradas para as covariáveis, a distribuição das estatísticas ajustadas está melhor representada pela distribuição normal padrão do que a estatística $R$, pois os tamanhos empíricos dos testes baseados nas estatísticas ajustadas estão mais próximos dos níveis nominais. Por exemplo, para covariáveis geradas da distribuição $t_{3}$ e um nível nominal $\alpha=5 \%$, os testes que usam as estatísticas $R, R^{*}, \bar{R}^{*}, \widehat{R}^{*}$ e $\widetilde{R}^{*}$ apresentaram os tamanhos empíricos 7,9\%, 5,4\%, $5,3 \%, 4,5 \%$ e $5 \%$, respectivamente. Enfim, quando as covariáveis são geradas das distribuições consideradas na Tabela 4.3, os desempenhos dos testes baseados nas estatísticas ajustadas são próximos.

Apresentamos na Tabela 4.4 os resultados da simulação de 100000 amostras de Monte Carlo, ou seja, 10 vezes mais réplicas do que as simulações anteriores. O uso de um número maior de réplicas permitirá avaliar os tamanhos empíricos dos testes para valores bem pequenos do nível nominal. Uma vez que as hipóteses estatísticas consideradas são $\mathrm{H}_{0}: \psi \leq 0$ e $\mathrm{H}_{1}: \psi>0$, os tamanhos empíricos dos testes foram obtidos baseando-se na porcentagem de estatísticas que ficaram acima dos quantis da distribuição normal padrão localizados na cauda à direita. Observa-se na Tabela 4.4 que no extremo da cauda à direita da distribuição (que corresponde a tomar níveis nominais bem pequenos), o teste baseado na estatística $R$ mostrou-se muito liberal. Por exemplo, quando $\alpha=0,1 \%$, a taxa de rejeição empírica do teste baseado nessa estatística é de $0,43 \%$, enquanto que os tamanhos empíricos dos testes baseados nas demais estatísticas são mais próximos de $0,1 \%$, principalmente a taxa de rejeição do teste que usa a estatística $\tilde{R}^{*}$. Em todas as situações, com exceção do caso em que $\alpha=50 \%$, há uma discrepância maior do teste baseado na estatística $R$ do que os testes que usam as outras estatísticas consideradas. 
Tabela 4.4: Tamanhos empíricos dos testes no modelo normal linear (4.1), para $p=4, n=20$ e covariáveis uniformes.

\begin{tabular}{cccccc}
\hline Níveis Nominais & $R$ & \multicolumn{1}{c}{$R^{*}$} & $\bar{R}^{*}$ & $\widehat{R}^{*}$ & $\widetilde{R}^{*}$ \\
\hline $50 \%$ & 49,95 & 49,95 & 49,95 & 49,96 & 49,95 \\
$15 \%$ & 18,76 & 15,80 & 15,89 & 14,59 & 15,25 \\
$10 \%$ & 13,73 & 10,76 & 10,79 & 9,56 & 10,27 \\
$5 \%$ & 8,03 & 5,61 & 5,52 & 4,73 & 5,25 \\
$3 \%$ & 5,48 & 3,48 & 3,38 & 2,75 & 3,22 \\
$1 \%$ & 2,38 & 1,22 & 1,11 & 0,89 & 1,09 \\
$0,5 \%$ & 1,39 & 0,61 & 0,56 & 0,41 & 0,55 \\
$0,1 \%$ & 0,43 & 0,15 & 0,11 & 0,07 & 0,12 \\
$0,05 \%$ & 0,26 & 0,07 & 0,05 & 0,05 & 0,07 \\
$0,01 \%$ & 0,08 & 0,02 & 0,01 & 0,01 & 0,01 \\
\hline \hline
\end{tabular}

A Figura 4.1 apresenta o gráfico das discrepâncias relativas entre os níveis descritivos ("p-values") dos testes e os respectivos níveis descritivos assintóticos. Mais precisamente, denotando por $S$ a estatística do teste $\left(R, R^{*}, \bar{R}^{*}, \widehat{R}^{*}\right.$ ou $\widetilde{R}^{*}$, conforme o caso), a discrepância relativa acima mencionada, para um valor $z$ observado da estatística $S$, é definida por

$$
\frac{P(S>z)-P(Z>z)}{P(Z>z)}
$$

em que $Z \sim \mathrm{N}(0,1)$. 


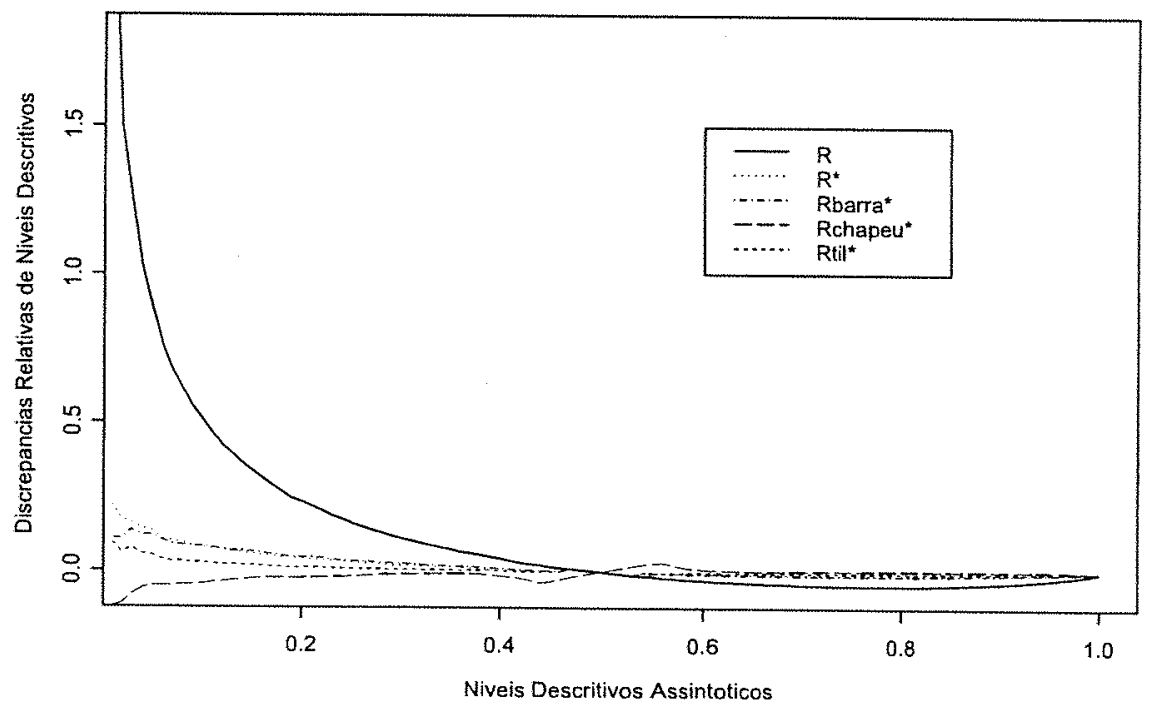

Figura 4.1: Gráfico das discrepâncias relativas para o modelo normal linear (4.1) com $n=20, p=4$ e covariáveis geradas da distribuição $\mathcal{U}(0,1)$.

Na Figura 4.1 podemos observar claramente que, quando se caminha em direção ao extremo da cauda à direita da distribuição normal padrão, as discrepâncias relativas para o teste baseado na estatística $R$ tendem a crescer rapidamente. A discrepância é bem menor para os outros testes.

Agora vamos considerar resultados numéricos para os modelos de regressão normais não lineares (4.2) e (4.3). Conforme mencionado anteriormente, para o caso não linear, podemos obter apenas as estatísticas $R, \bar{R}^{*}, \widehat{R}^{*}$ e $\widetilde{R}^{*}$. Os tamanhos dos testes foram obtidos para os tamanhos amostrais $n=10,20$ e 30, fixando a dimensão do vetor $\beta$ em $p=4$ e as covariáveis geradas independentemente a partir da distribuição $\mathcal{U}(0,1)$. Depois, mostraremos os resultados da simulação quando fixamos o tamanho amostral em $n=20$, a dimensão do vetor $\beta$ em $p=2,5$ e 8 e as covariáveis geradas da distribuição $\mathcal{U}(0,1)$. Por fim, estarão os resultados da simulação quando é fixado 
o tamanho amostral $(n=20)$, a dimensão de $\beta(p=4)$ e as covariáveis geradas de diferentes distribuições. Nesta etapa, a primeira coluna da matriz $X$, de dimensão $n \times(p+1)$ é de uns e as demais colunas são geradas a partir das distribuições $t_{3}, t_{10}$ e $\chi_{5}^{2}$, sem nenhuma transformação.

Inicialmente temos os resultados dos tamanhos dos testes para o modelo de regressão normal não linear (4.2) nas Tabelas 4.5, 4.6 e 4.7. Em seguida, apresentamos na Tabela 4.8 os resultados de simulação de 100000 amostras de Monte Carlo, ou seja, 10 vezes mais réplicas do que as simulações anteriores, conforme foi realizado para o caso linear.

$\mathrm{Na}$ Tabela 4.5 são apresentados os resultados de dez mil amostras geradas para cada combinação dos tamanhos amostrais $(n=10,20$ e 30), a dimensão do vetor $\beta$ foi fixado em $p=4$ e as covariáveis geradas da distribuição $\mathcal{U}(0,1)$.

Tabela 4.5: Tamanhos empíricos dos testes no modelo normal não linear (4.2), para $n=10,20$ e 30, $p=4$ e covariáveis uniformes.

\begin{tabular}{c|rrrr|rrrr|rrrr}
\hline \hline \multicolumn{1}{c|}{$n=10$} & \multicolumn{1}{c}{$n=20$} \\
\hline \hline Nominal & \multicolumn{1}{c|}{$R$} & $\bar{R}^{*}$ & $\widehat{R}^{*}$ & $\widetilde{R}^{*}$ & \multicolumn{1}{c}{$R$} & $\widetilde{R}^{*}$ & $\widehat{R}^{*}$ & $\widetilde{R}^{*}$ & $R$ & $\bar{R}^{*}$ & $\widehat{R}^{*}$ & $\widetilde{R}^{*}$ \\
\hline $10 \%$ & 19,2 & 11,3 & 11,1 & 11,9 & 13,8 & 10,8 & 10,2 & 10,4 & 11,8 & 10,4 & 9,7 & 9,9 \\
$5 \%$ & 13,4 & 5,3 & 6,5 & 6,2 & 8,2 & 5,8 & 5,0 & 5,5 & 6,6 & 5,5 & 4,9 & 5,1 \\
$1 \%$ & 5,4 & 1,0 & 2,1 & 1,5 & 2,5 & 1,2 & 0,8 & 1,2 & 1,6 & 0,9 & 0,8 & 0,8 \\
$0,5 \%$ & 3,8 & 0,5 & 1,6 & 0,9 & 1,3 & 0,5 & 0,4 & 0,5 & 0,8 & 0,5 & 0,4 & 0,4 \\
\hline \hline
\end{tabular}

Observa-se que o teste baseado na estatística $R$ mostrou-se liberal para todos os tamanhos amostrais considerados, no sentido de que tende a rejeitar a hipótese nula com uma frequência bem maior do que a indicada pelo correspondente nível nominal. Nota-se uma necessidade maior de ajustes para a estatística $R$ quando o tamanho amostral é pequeno. Por exemplo, para $n=10$ e um nível de $5 \%$, os tamanhos 
empíricos dos testes baseados em $R, \bar{R}^{*}, \widehat{R}^{*}$ e $\widetilde{R}^{*}$ são $13,4 \%, 5,3 \%, 6,5 \%$ e $6,2 \%$, respectivamente. Entre as estatísticas ajustadas, destaca-se com melhor desempenho a estatística $\bar{R}^{*}$, pois as taxas de rejeição observadas baseadas nessa estatística são mais próximas dos níveis nominais considerados. Evidentemente, ajustes à estatística $R$ têm menor efeito quando o tamanho amostral é grande.

Considere agora o caso em que é fixado o tamanho amostral, $n=20$, variada a dimensão do vetor $\beta$ em $p=2,5$ e 8 e as covariáveis são geradas da distribuição $\mathcal{U}(0,1)$. Os resultados são apresentados na Tabela 4.6 .

Tabela 4.6: Tamanhos empiricos dos testes no modelo normal não linear (4.2), para $p=2,5$ e $8, n=20$ e covariáveis uniformes.

\begin{tabular}{c|rrrr|rrrr|rrrr}
\hline \hline \multicolumn{1}{c|}{$p=2$} & \multicolumn{1}{c}{$p=5$} \\
\hline \hline Nominal & \multicolumn{1}{c|}{$R$} & $\bar{R}^{*}$ & $\widehat{R}^{*}$ & $\widetilde{R}^{*}$ & \multicolumn{1}{c}{$R$} & $\bar{R}^{*}$ & $\widehat{R}^{*}$ & $\widetilde{R}^{*}$ & $R$ & $\bar{R}^{*}$ & $\widehat{R}^{*}$ & $\widetilde{R}^{*}$ \\
\hline $10 \%$ & 12,2 & 11,2 & 10,2 & 10,4 & 14,9 & 11,2 & 9,8 & 10,8 & 17,0 & 11,4 & 9,0 & 11,3 \\
$5 \%$ & 6,7 & 5,7 & 5,1 & 5,3 & 9,1 & 6,0 & 4,6 & 5,8 & 11,3 & 5,9 & 4,4 & 5,9 \\
$1 \%$ & 1,6 & 1,1 & 0,9 & 1,0 & 2,7 & 1,1 & 0,8 & 1,1 & 4,3 & 1,4 & 1,1 & 1,6 \\
$0,5 \%$ & 0,8 & 0,6 & 0,4 & 0,5 & 1,7 & 0,5 & 0,4 & 0,5 & 3,0 & 0,8 & 0,7 & 0,9 \\
\hline \hline
\end{tabular}

Observando a Tabela 4.6, nota-se que os tamanhos empíricos dos testes baseados nas estatísticas $\bar{R}^{*}, \widehat{R}^{*}$ e $\widetilde{R}^{*}$ tendem a ser mais próximos dos correspondentes níveis nominais do que os tamanhos empíricos dos testes usando $R$. O teste baseado em $R$ se mostrou liberal, apresentando taxas de rejeição observadas acima dos níveis nominais correspondentes. Como esperado, a estatística $R$ não tem boa aproximação pela distribuição normal padrão quando o número de parâmetros de perturbação $(p+1)$ é alto relativamente ao tamanho da amostra considerado. Assim, os ajustes à estatística $R$ são mais necessários para valores altos de $p$ (dimensão do vetor $\beta$ ). Por exemplo, para $p=8$ e um nível de $5 \%$, os tamanhos empíricos dos testes baseados em $R, \bar{R}^{*}, \widehat{R}^{*}$ e $\widetilde{R}^{*}$ são $11,3 \%, 5,9 \%, 4,4 \%$ e $5,9 \%$, respectivamente. Para as situações 
apresentadas nessa tabela, a estatística que se destacou com melhor desempenho foi $\widehat{R}^{*}$, pois as taxas de rejeição observadas para o teste que usa essa estatística são mais próximas (ou abaixo) dos níveis nominais considerados.

A Tabela 4.7 apresenta os resultados de dez mil amostras de tamanho $n=20$, com a dimensão do vetor $\beta$ fixado em $p=4$ e as covariáveis geradas segundo diferentes distribuições.

Tabela 4.7: Tamanhos empíricos dos testes no modelo normal não linear (4.2), para $p=4, n=20$ e covariáveis com distribuição $t_{3}, t_{10}$ e $\chi_{5}^{2}$.

\begin{tabular}{c|rrrr|rrrr|rrrr}
\hline \hline \multicolumn{1}{c|}{$t_{3}$} & \multicolumn{1}{c}{$t_{10}$} & \multicolumn{4}{c}{$\chi_{5}^{2}$} \\
\hline \hline Nominal & \multicolumn{1}{c|}{$R$} & $\widetilde{R}^{*}$ & $\widehat{R}^{*}$ & \multicolumn{1}{c}{$\widetilde{R}^{*}$} & \multicolumn{1}{c}{$R$} & $\bar{R}^{*}$ & $\widehat{R}^{*}$ & $\widetilde{R}^{*}$ & $R$ & $\bar{R}^{*}$ & $\widehat{R}^{*}$ & $\widetilde{R}^{*}$ \\
\hline $10 \%$ & 14,5 & 11,6 & 10,2 & 10,9 & 13,7 & 10,8 & 9,6 & 10,3 & 13,7 & 11,1 & 9,8 & 10,6 \\
$5 \%$ & 8,7 & 6,0 & 5,1 & 5,6 & 8,1 & 5,4 & 4,5 & 5,1 & 8,0 & 5,6 & 5,3 & 5,2 \\
$1 \%$ & 2,5 & 1,1 & 1,1 & 1,1 & 2,2 & 1,0 & 0,8 & 0,9 & 2,2 & 1,1 & 1,1 & 1,1 \\
$0,5 \%$ & 1,4 & 0,6 & 0,5 & 0,6 & 1,3 & 0,5 & 0,4 & 0,4 & 1,3 & 0,5 & 0,6 & 0,6 \\
\hline \hline
\end{tabular}

Observa-se na Tabela 4.7 que o teste baseado na estatística $R$ mostrou-se liberal, apresentando taxas de rejeição acima dos correspondentes níveis nominais. Observamos ainda que, para a grande maioria dos casos, as distribuições de $\bar{R}^{*}, \widehat{R}^{*}$ e $\widetilde{R}^{*}$ estão melhor representadas pela distribuição normal padrão do que a estatística $R$. Além disso, o teste que usa a estatística $\widehat{R}^{*}$, baseada em covariâncias empíricas, tende a ser melhor, no sentido de que apresenta tamanhos empíricos mais próximos (ou abaixo) dos níveis nominais. Por exemplo, para covariáveis geradas da distribuição $t_{3}$ e um nível de $5 \%$, os tamanhos empíricos dos testes baseados em $R, \bar{R}^{*}, \widehat{R}^{*}$ e $\widetilde{R}^{*}$ foram $8,7 \%, 6 \%, 5,1 \%$ e $5,6 \%$, respectivamente.

Apresentamos na Tabela 4.8 os resultados de simulação de cem mil amostras de Monte Carlo, ou seja, 10 vezes mais réplicas do que as simulações anteriores, com o objetivo de avaliar os tamanhos empíricos dos testes para valores bem pequenos do 
nível nominal. Os resultados apresentados nessa tabela são referentes ao caso não linear, utilizando o modelo (4.2), com amostras de tamanho $n=20$, a dimensão do vetor $\beta$ fixado em $p=4$ e covariáveis obtidas de uma distribuição $\mathcal{U}(0,1)$.

Tabela 4.8: Tamanhos empiricos dos testes no modelo normal não linear (4.2), para $p=4, n=20$ e covariáveis uniformes.

\begin{tabular}{ccccc}
\hline \hline Níveis Nominais & \multicolumn{1}{c}{$R$} & \multicolumn{1}{c}{$\bar{R}^{*}$} & $\widehat{R}^{*}$ & $\widetilde{R}^{*}$ \\
\hline $50 \%$ & 49,93 & 51,69 & 51,77 & 51,77 \\
$15 \%$ & 18,84 & 16,17 & 15,06 & 15,60 \\
$10 \%$ & 13,64 & 10,85 & 9,85 & 10,32 \\
$5 \%$ & 7,91 & 5,59 & 4,80 & 5,30 \\
$3 \%$ & 5,38 & 3,43 & 2,86 & 3,27 \\
$1 \%$ & 2,31 & 1,17 & 0,91 & 1,15 \\
$0,5 \%$ & 1,37 & 0,61 & 0,46 & 0,61 \\
$0,1 \%$ & 0,42 & 0,16 & 0,13 & 0,18 \\
$0,05 \%$ & 0,27 & 0,09 & 0,07 & 0,10 \\
$0,01 \%$ & 0,09 & 0,03 & 0,02 & 0,04 \\
\hline \hline
\end{tabular}

Notamos que, para níveis nominais bem pequenos, as taxas de rejeição do teste baseado na estatística $R$ estão bem acima dos níveis nominais considerados. Por exemplo, para $\alpha=0,1 \%$, os tamanhos empíricos dos testes baseados em $R, \bar{R}^{*}, \widehat{R}^{*}$ e $\widetilde{R}^{*}$ são $0,42 \%, 0,16 \%$ e $0,13 \%$ e $0,18 \%$, respectivamente. Para todos os níveis nominais, com excȩão do caso em que $\alpha=50 \%$, há uma discrepância maior dos testes quando se usa a estatística $R$ do que quando se utilizam as demais estatísticas consideradas. Em geral, os testes baseados nas estatísticas ajustadas apresentaram desempenhos mais satisfatórios do que os testes que usam a estatística $R$.

A Figura 4.2 apresenta o gráfico das discrepâncias relativas entre os níveis des- 
critivos ("p-values") dos testes e os respectivos níveis assintóticos para o caso não linear, utilizando o modelo (4.2).

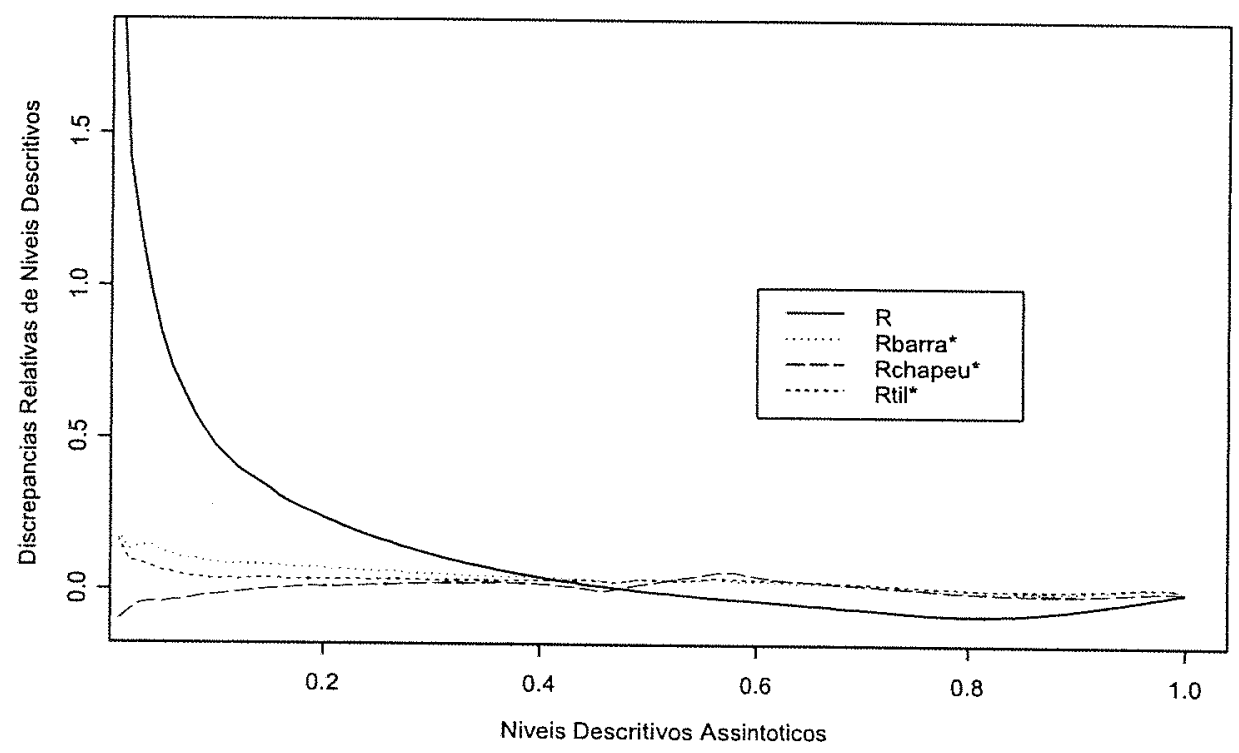

Figura 4.2: Gráfico das discrepâncias relativas para o modelo normal não linear (4.2) com $n=20, p=4$ e covariáveis geradas da distribuição $\mathcal{U}(0,1)$.

A Figura 4.2 mostra que quando se caminha em direção ao extremo da cauda à direita da distribuição normal padrão, a discrepância relativa de níveis descritivos do teste baseado na estatística $R$ tende a crescer rapidamente e é bem menor para os testes baseados nas estatísticas ajustadas.

Finalmente, apresentaremos os resultados de simulação para o outro caso não linear, utilizando o modelo (4.3). Nessa situação, também foram considerados os resultados numéricos baseados nas estatísticas $R, \bar{R}^{*}, \widehat{R}^{*}$ e $\widetilde{R}^{*}$. Da mesma forma que para o caso linear e o não linear apresentados anteriormente, foram simuladas dez mil amostras e obtidos os tamanhos empíricos dos testes nas seguintes situações: (i) tamanhos amostrais $n=10,20$ e 30 , dimensão do vetor $\beta$ fixado em $p=4$ e as 
covariáveis geradas independentemente a partir da distribuição $\mathcal{U}(0,1)$; (ii) tamanho amostral, $n=20$, dimensão do vetor $\beta$ fixado em $p=2,5$ e 8 e as covariáveis geradas da distribuição $\mathcal{U}(0,1)$ e (iii) tamanho amostral $n=20$, dimensão de $\beta$ tomado como $p=4$ e as covariáveis geradas de diferentes distribuições $\left(t_{3}, t_{10}\right.$ e $\left.\chi_{5}^{2}\right)$, sem nenhuma transformação.

Os casos (i)-(iii) avaliados numericamente serão apresentados através das Figuras $4.3,4.4$ e 4.5, respectivamente. Nessas figuras apresentam-se os gráficos de quantis ("q-q plot") para as estatísticas $R, \bar{R}^{*}, \widehat{R}^{*}$ e $\widetilde{R}^{*}$. Os gráficos foram construídos com os valores ordenados de cada estatística contra os quantis correspondentes da distribuição normal padrão. Se a distribuição da estatística analisada apresenta uma boa aproximação pela distribuição normal padrão, espera-se que este gráfico seja próximo de uma reta, formando um ângulo de $45^{\circ} \mathrm{com}$ o eixo horizontal. Para facilitar esta avaliação, construímos uma reta considerada ideal, em que os pontos deveriam estar, caso houvesse uma perfeita concordância entre os quantis amostrais da distribuição da estatística do teste analisado e os quantis correspondentes da distribuição normal padrão.

Podemos observar nas Figuras 4.3, 4.4 e 4.5 que os resultados baseados no modelo (4.3) foram muito parecidos com os resultados baseados no modelo (4.2), no sentido de que em todas as situações os testes baseados nas estatísticas ajustadas apresentaram melhor desempenho do que o teste baseado na estatística $R$. Por exemplo, na Figura 4.3, o caso extremo em que o tamanho amostral é pequeno, ou seja, quando $n=10$, os quantis das estatísticas modificadas estão bem mais próximos dos quantis da distribuição normal padrão do que os quantis da estatística $R$. Outro caso extremo, apresentado na Figura 4.4, em que os quantis das estatísticas ajustadas são mais próximos dos quantis da normal padrão do que os quantis de $R$, é quando o número de parâmetros de perturbação é alto, ou seja, quando a dimensão 
do vetor $\beta$ foi fixada em $p=8$. Por fim, na Figura 4.5, que apresenta os "q - qplots", quando são geradas covariáveis de diferentes distribuições, temos também que os quantis baseados nas estatísticas ajustadas mostraram-se mais próximos dos quantis da distribuição normal padrão do que os quantis baseados em $R$.
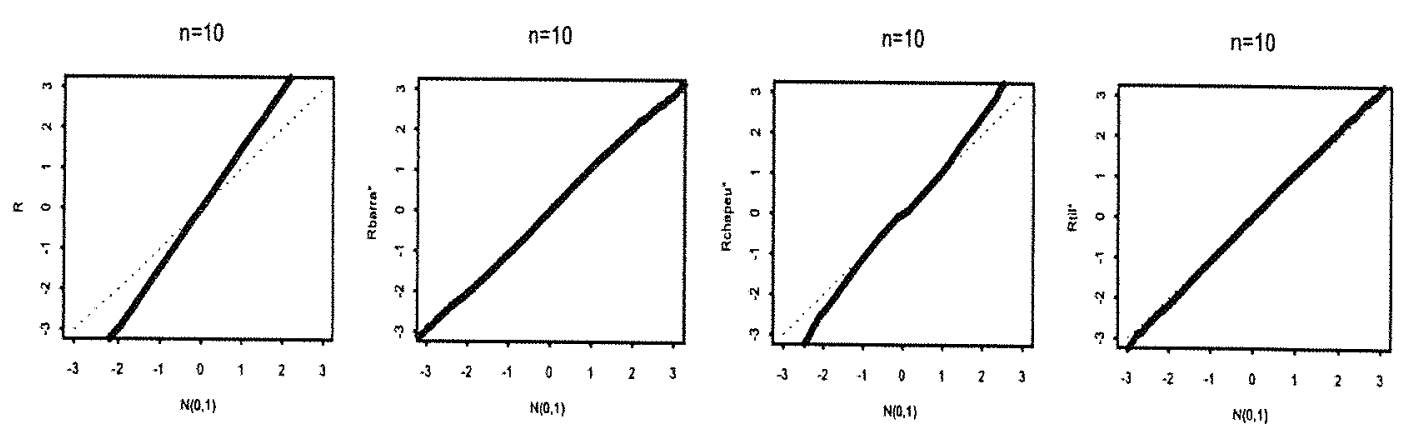

$n=20$
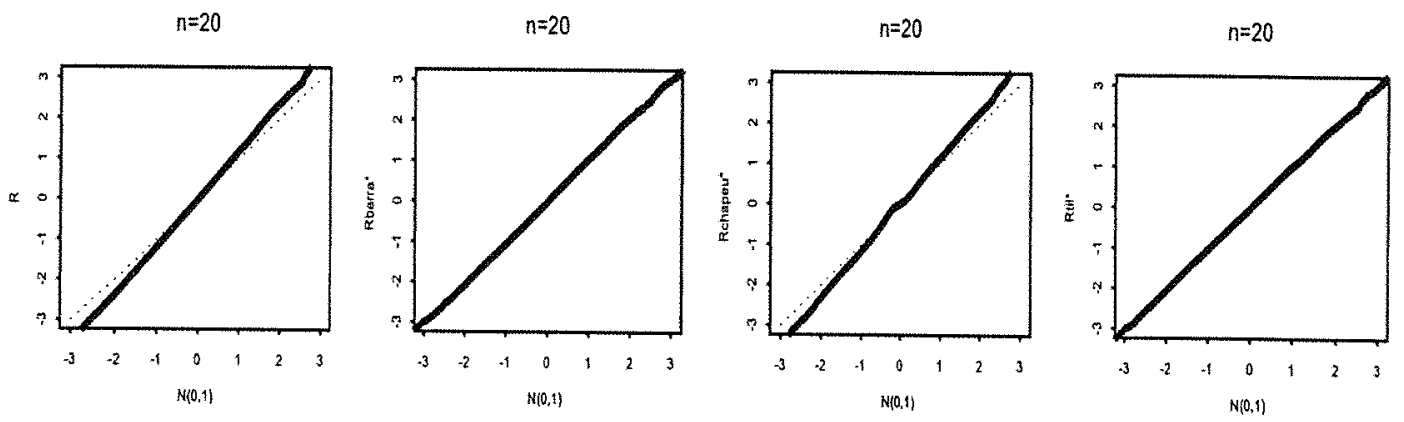

$n=30$
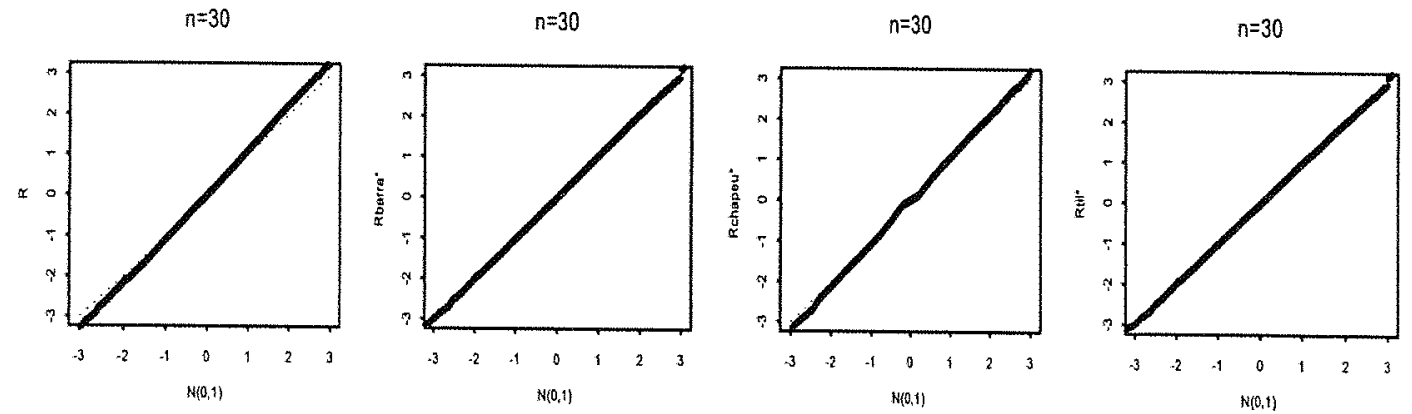

Figura 4.3: Gráficos q-q plot para as estatísticas $R, \bar{R}^{*}, \widehat{R}^{*}$ e $\widetilde{R}^{*}$ utilizando o modelo normal não linear (4.3) com $n=10$, 20 e 30, $p=4$ e covariáveis uniforme. 

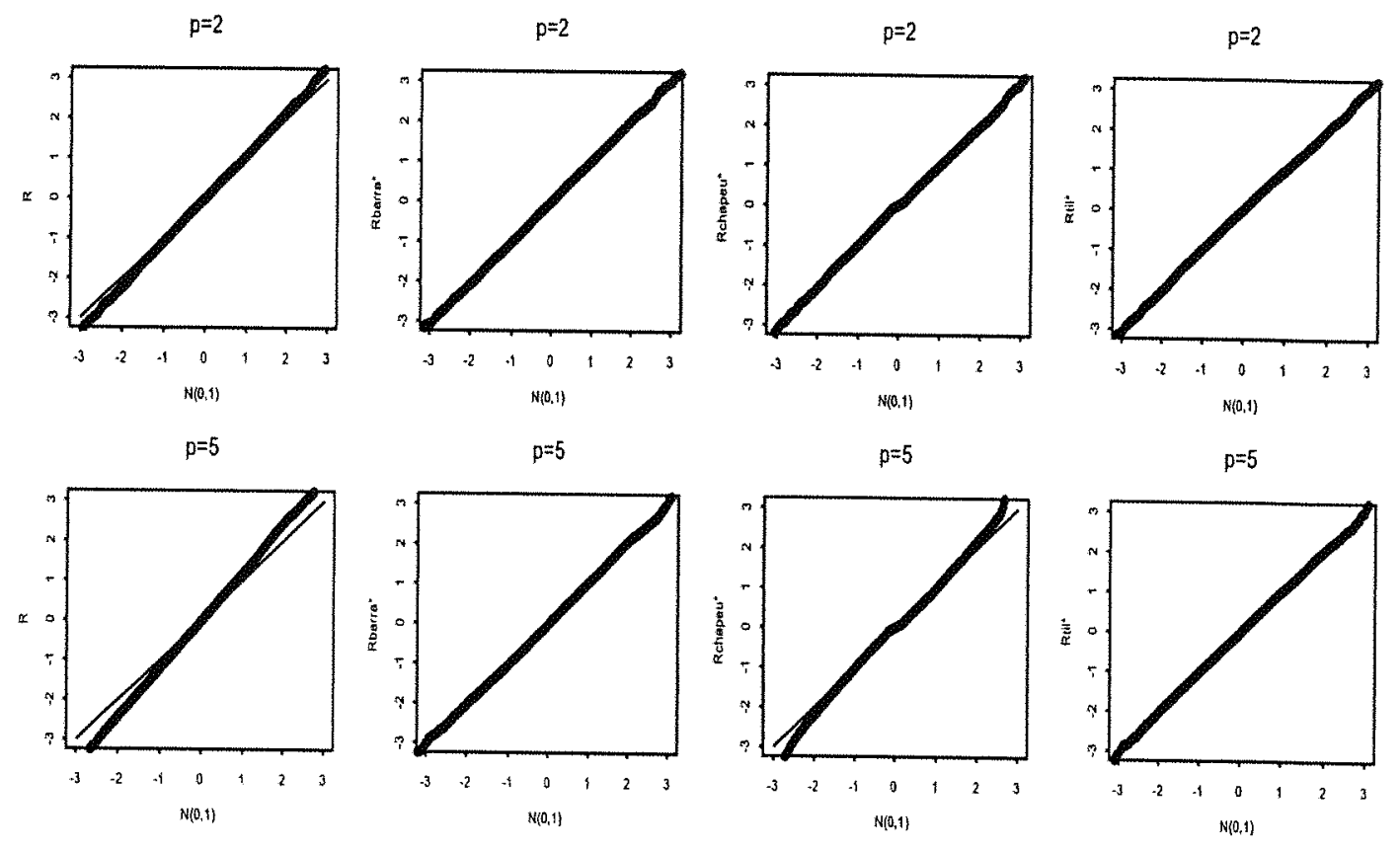

$p=8$
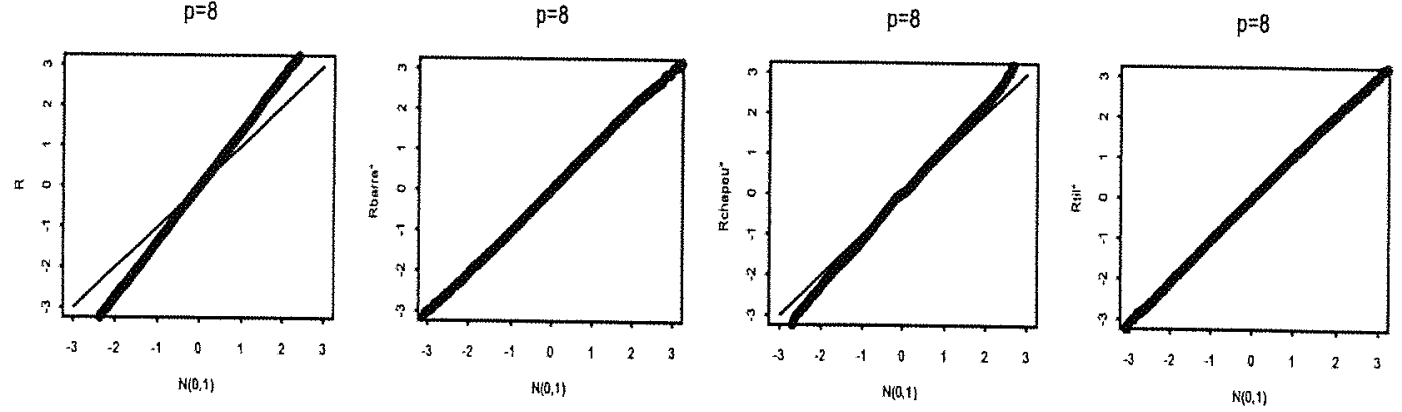

Figura 4.4: Gráficos $q$-q plot para as estatísticas $R, \bar{R}^{*}, \widehat{R}^{*}$ e $\widetilde{R}^{*}$ utilizando o modelo normal não linear (4.3) com $n=20, p=2,5$ e 8 e covariáveis uniforme. 
13

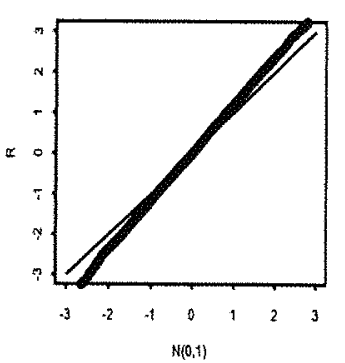

t10

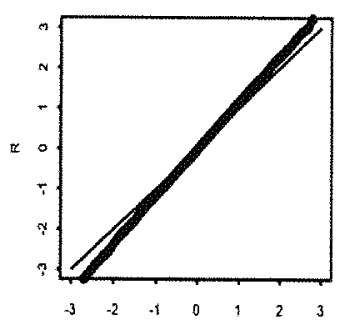

N\{0.1\}

QQ5

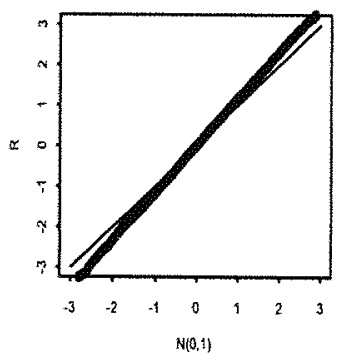

t3

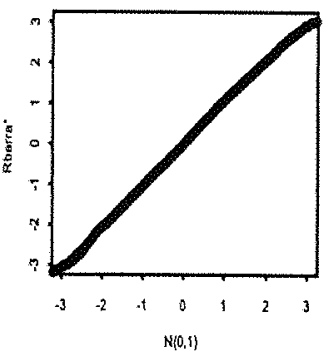

110

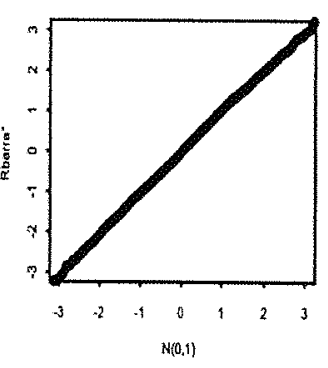

QQ5

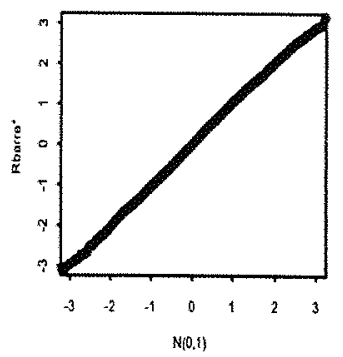

$\mathrm{t} 3$

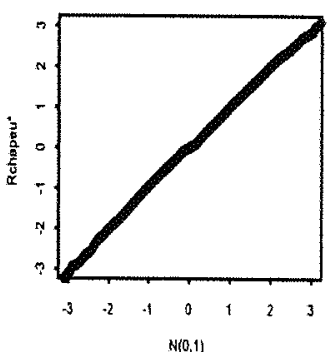

t10

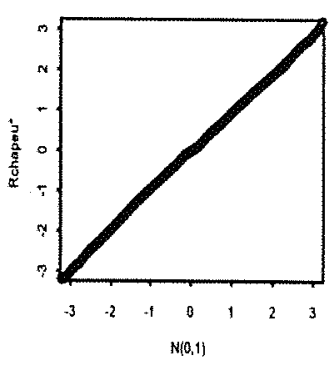

QQ5

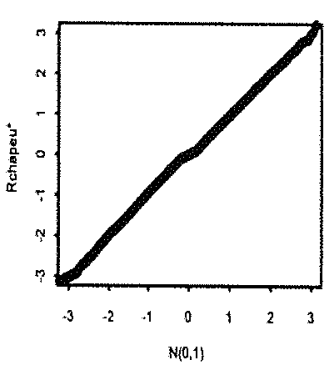

13

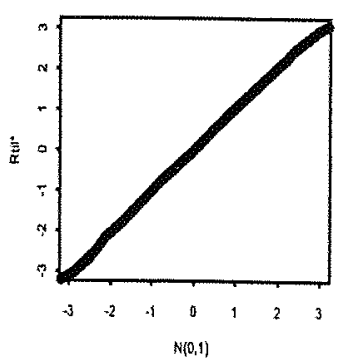

110

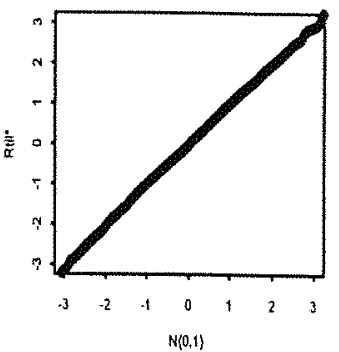

QQ5

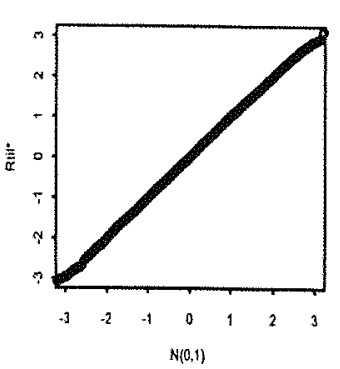

Figura 4.5: Gráficos q-q plot para as estatísticas $R, \bar{R}^{*}, \widehat{R}^{*}$ e $\widetilde{R}^{*}$ utilizando o modelo normal não linear (4.3) com $n=20, p=4$ e covariáveis $t_{3}, t_{10} e \chi_{5}^{2}$. 


\subsection{Poder dos Testes}

Nessa seção avaliamos o poder dos testes em estudo sob diversas situações. Para tal, realizamos simulações sob a hipótese alternativa em que $\psi>0$. Conforme mencionado no Capítulo 3, para o caso linear, $R^{*}$ e $R_{0}^{*}$ são iguais e, por isso, mostraremos apenas $R^{*}$. Será desconsiderado o poder do teste baseado na estatística $R$, pois nas simulações de tamanho dos testes, esse teste mostrou-se liberal, ou seja, apresentou, sob $H_{0}$, taxas de rejeição superiores aos níveis de significância nominais. Apresentaremos os testes baseados nas estatísticas que tiveram desempenhos semelhantes para que seja possível compará-los em termos de poder. Sendo assim, mostraremos o poder dos testes referentes às estatísticas $R^{*}, \bar{R}^{*}, \widehat{R}^{*}$ e $\widetilde{R}^{*}$ no caso linear e, no caso não linear, $\bar{R}^{*}, \widehat{R}^{*}$ e $\widetilde{R}^{*}$.

Em primeiro lugar, apresentamos os resultados relativos ao poder dos testes no modelo de regressão normal linear, tomando-se $\psi$ variando entre 0,2 e 1 , com incrementos iguais a 0,2 . O poder dos testes será avaliado sob diversas situações, mas sempre considerando $\alpha=5 \%$. Primeiramente fixamos a dimensão do vetor $\beta \mathrm{em}$ $p=4$, variamos o tamanho amostral em $n=10,20$ e 30 e geramos covariáveis da distribuição uniforme. Depois, fixamos o tamanho amostral em $n=20$, variamos a dimensão do vetor $\beta$ em $p=2,5$ e 8 e geramos covariáveis da distribuição uniforme. Por fim, fixamos o tamanho amostral em $n=20$, a dimensão do vetor $\beta$ em $p=4 \mathrm{e}$ geramos covariáveis das distribuições $t_{3}, t_{10}$ e $\chi_{5}^{2}$. Vale lembrar que a matriz $X$, neste caso linear, foi transformada de tal forma que $\sum_{i=1}^{n} x_{i}=0$.

A Tabela 4.9 apresenta os resultados referentes ao poder dos testes quando é fixada a dimensão do vetor $\beta$ em $p=4$, variado o tamanho amostral em $n=10,20$ e 30 e as covariáveis são uniformes. Observando a Tabela 4.9, os poderes dos testes baseados em $R^{*}, \bar{R}^{*}$ e $\widetilde{R}^{*}$ mostraram-se ligeiramente maiores que aquele baseado em $\widehat{R}^{*}$. O 
teste baseado na estatística $\bar{R}^{*}$, em geral, foi o que apresentou melhor desempenho, pois mostrou-se com maior poder. Por outro lado, o teste que se destacou com poder razoavelmente mais baixo que os demais foi o teste que usa $\widehat{R}^{*}$, a estatística baseada em covariâncias empíricas proposta por Severini (1999). Como esperado, observa-se que à medida que o tamanho amostral aumenta, os podereres dos testes também aumentam. Foram omitidos os poderes dos testes que usam as estatísticas $R^{*}$ e $\widehat{R}^{*}$, quando o tamanho amostral é $n=10$, pois no estudo dos tamanhos dos testes, os testes baseados nessas estatísticas mostraram-se liberais (ver Tabela 4.1).

Tabela 4.9: Poder dos testes no modelo normal linear (4.1) para

\begin{tabular}{ccccccc}
$n=10,20$ & $e$ & $30, p=4$ & e covariáveis com distribuição unifor \\
\hline & & & \multicolumn{6}{c}{$\psi$} \\
\cline { 3 - 8 }$n$ & Estatística & 0,2 & 0,4 & 0,6 & 0,8 & 1,0 \\
\hline \hline 10 & $\bar{R}^{*}$ & 15,33 & 33,34 & 55,45 & 76,85 & 90,62 \\
& $\widetilde{R}^{*}$ & 16,35 & 33,62 & 54,94 & 75,07 & 88,84 \\
\hline 20 & $R^{*}$ & 22,24 & 53,43 & 82,58 & 96,38 & 99,57 \\
& $\bar{R}^{*}$ & 22,65 & 55,19 & 84,20 & 96,88 & 99,73 \\
& $\widehat{R}^{*}$ & 19,45 & 48,93 & 78,33 & 94,02 & 98,86 \\
& $\widetilde{R}^{*}$ & 21,47 & 52,89 & 82,41 & 96,44 & 99,60 \\
\hline \multirow{2}{*}{30} & $R^{*}$ & 28,84 & 68,79 & 94,17 & 99,64 & 99,99 \\
& $\bar{R}^{*}$ & 29,65 & 70,31 & 94,74 & 99,75 & 99,99 \\
& $\widehat{R}^{*}$ & 27,79 & 67,34 & 93,39 & 99,55 & 99,99 \\
& $\widetilde{R}^{*}$ & 28,49 & 68,70 & 94,18 & 99,68 & 99,99 \\
\hline \hline
\end{tabular}

A Tabela 4.10 apresenta o poder dos testes quando o tamanho amostral é 20, as dimensões do vetor $\beta$ são $p=2,5$ e 8 e as covariáveis são uniformes. Podemos observar que, em geral, os testes apresentaram desempenhos próximos em termos 
de poder, com exceção do teste baseado na estatística $\widehat{R}^{*}$. O teste baseado nessa estatística é menos poderoso, porque mostrou-se conservativo (ver Tabela 4.2) e, isto implica, em geral, em teste com menos poder. O teste baseado na estatística $\vec{R}^{*}$, para as situações apresentadas nessa tabela, é o que apresentou poder mais alto. Foi omitido o poder do teste que usa a estatística $R^{*}$, quando a dimensão do vetor $\beta$ é fixada em $p=8$, pois no estudo dos tamanhos dos testes, o teste baseado nessa estatística mostrou-se liberal (ver Tabela 4.2).

Tabela 4.10: Poder dos testes no modelo normal linear (4.1) para

\begin{tabular}{|c|c|c|c|c|c|c|}
\hline \multirow[b]{2}{*}{$p$} & \multirow[b]{2}{*}{ Estatística } & \multicolumn{3}{|c|}{$\psi$} & \multirow[b]{2}{*}{0,8} & \multirow[b]{2}{*}{1,0} \\
\hline & & 0,2 & 0,4 & 0,6 & & \\
\hline \multirow[t]{4}{*}{2} & $R^{*}$ & 21,45 & 52,97 & 82,34 & 96,39 & 99,53 \\
\hline & $\bar{R}^{*}$ & 22,64 & 55,75 & 84,55 & 96,99 & 99,71 \\
\hline & $\widehat{R}^{*}$ & 20,86 & 52,37 & 82,14 & 96,18 & 99,56 \\
\hline & $\widetilde{R}^{*}$ & 21,20 & 52,88 & 82,48 & 96,45 & 99,57 \\
\hline \multirow[t]{4}{*}{5} & $R^{*}$ & 22,76 & 54,07 & 82,81 & 96,29 & 99,55 \\
\hline & $\bar{R}^{*}$ & 22,77 & 54,99 & 84,11 & 96,79 & 99,72 \\
\hline & $\widehat{R}^{*}$ & 17,78 & 45,71 & 75,50 & 91,96 & 97,93 \\
\hline & $\widetilde{R}^{*}$ & 21,88 & 53,33 & 82,54 & 96,33 & 99,59 \\
\hline \multirow[t]{3}{*}{8} & $\bar{R}^{*}$ & 23,30 & 54,96 & 84,03 & 96,80 & 99,72 \\
\hline & $\widehat{R}^{*}$ & 12,87 & 32,56 & 57,72 & 79,72 & 92,81 \\
\hline & $\widetilde{R}^{*}$ & 23,07 & 54,26 & 83,22 & 96,44 & 99,60 \\
\hline
\end{tabular}

A Tabela 4.11 apresenta o poder dos testes para a situação em que o tamanho amostral é $n=20$, a dimensão do vetor $\beta$ é $p=4$ e as covariáveis são geradas segundo as distribuições $t_{3}, t_{10}$ e $\chi_{5}^{2}$. Observa-se que o teste baseado na estatística 
$\vec{R}^{*}$ é ligeiramente mais poderoso do que os testes baseados nas demais estatísticas ajustadas, independentemente das covariáveis consideradas. Por outro lado o teste baseado na estatística $\widehat{R}^{*}$ é o menos poderoso. Note que os testes baseados nas estatísticas $R^{*}$ e $\widetilde{R}^{*}$ apresentaram poderes muito parecidos.

Tabela 4.11: Poder dos testes no modelo normal linear (4.1), para $n=20, p=4$ e covariáveis com distribuição $t_{3}, t_{10}$ e $\chi_{5}^{2}$.

\begin{tabular}{|c|c|c|c|c|c|c|}
\hline \multirow[b]{2}{*}{ Dist. } & \multirow[b]{2}{*}{ Estatística } & \multicolumn{3}{|c|}{$\psi$} & \multirow[b]{2}{*}{0,8} & \multirow[b]{2}{*}{1,0} \\
\hline & & 0,2 & 0,4 & 0,6 & & \\
\hline \multirow[t]{4}{*}{$t_{3}$} & $R^{*}$ & 22,40 & 53,90 & 82,66 & 96,29 & 99,61 \\
\hline & $\bar{R}^{*}$ & 22,74 & 55,36 & 84,16 & 96,86 & 99,72 \\
\hline & $\widehat{R}^{*}$ & 17,77 & 44,36 & 71,58 & 89,14 & 97,14 \\
\hline & $\widetilde{R}^{*}$ & 21,53 & 53,15 & 82,53 & 96,39 & 99,64 \\
\hline \multirow[t]{4}{*}{$t_{10}$} & $R^{*}$ & 22,11 & 53,91 & 82,79 & 96,19 & 99,59 \\
\hline & $\bar{R}^{*}$ & 22,48 & 55,46 & 84,33 & 96,88 & 99,72 \\
\hline & $\widehat{R}^{*}$ & 17,06 & 48,20 & 77,46 & 93,21 & 98,44 \\
\hline & $\widetilde{R}^{*}$ & 21,30 & 53,30 & 82,72 & 96,26 & 99,62 \\
\hline \multirow[t]{4}{*}{$\chi_{5}^{2}$} & $R^{*}$ & 22,20 & 53,66 & 82,88 & 96,47 & 99,55 \\
\hline & $\bar{R}^{*}$ & 22,73 & 55,25 & 84,30 & 96,93 & 99,72 \\
\hline & $\widehat{R}^{*}$ & 17,56 & 47,51 & 76,50 & 93,24 & 98,32 \\
\hline & $\widetilde{R}^{*}$ & 21,54 & 53,16 & 82,78 & 96,46 & 99,59 \\
\hline
\end{tabular}

Agora, apresentaremos os resultados relativos aos poderes dos testes no modelo normal não linear (4.2), para variações do parâmetro $\psi$ adequadas e $\alpha=5 \%$. Os resultados se encontram nas Tabelas $4.12-4.16$.

A Tabela 4.12 apresenta o poder dos testes para os tamanhos amostrais $n=10,20$ e 30 , a dimensão do vetor $\beta$ fixado em $p=4$ e as covariáveis geradas segundo uma 
distribuição uniforme. Observando essa tabela, notamos que os testes baseados nas estatísticas $\bar{R}^{*}$ e $\widetilde{R}^{*}$ são, em geral, mais poderosos que o teste baseado na estatística $\widehat{R}^{*}$.

Tabela 4.12: Poder dos testes no modelo normal não linear (4.2), para $n=10,20$ e 30, $p=4$ e covariáveis com distribuição uniforme.

\begin{tabular}{cccccccc}
\hline \hline & & \multicolumn{6}{c}{$\psi$} \\
\cline { 3 - 7 }$n$ & Estatística & 0,2 & 0,6 & 1,0 & 1,4 & 1,8 & 2,2 \\
\hline \hline \multirow{2}{*}{10} & $\bar{R}^{*}$ & 7,94 & 17,41 & 39,31 & 74,80 & 98,14 & 100,00 \\
& $\widehat{R}^{*}$ & 10,85 & 19,56 & 38,51 & 68,77 & 93,81 & 99,80 \\
& $\widetilde{R}^{*}$ & 9,13 & 18,69 & 40,71 & 74,92 & 97,48 & 100,00 \\
\hline \multirow{2}{*}{20} & $\bar{R}^{*}$ & 10,04 & 30,37 & 73,22 & 98,89 & 100,00 & 100,00 \\
& $\widehat{R}^{*}$ & 8,39 & 26,41 & 67,53 & 97,56 & 99,99 & 100,00 \\
& $\widetilde{R}^{*}$ & 9,87 & 30,31 & 72,17 & 98,60 & 100,00 & 100,00 \\
\hline 30 & $\bar{R}^{*}$ & 10,45 & 39,48 & 87,52 & 99,91 & 100,00 & 100,00 \\
& $\widehat{R}^{*}$ & 9,54 & 37,37 & 85,75 & 99,87 & 100,00 & 100,00 \\
& $\widetilde{R}^{*}$ & 10,79 & 39,25 & 87,12 & 99,93 & 100,00 & 100,00 \\
\hline \hline
\end{tabular}

A Tabela 4.13 apresenta os poderes dos testes quando o tamanho amostral é $n=20$, as dimensões do vetor $\beta$ são $p=2,5$ e 8 e as covariáveis são geradas da distribuição uniforme. Observando essa tabela, nota-se que mesmo com a variação do número de parâmetros de perturbação $(p+1)$, o teste baseado na estatística $\widetilde{R}^{*}$ mostrou-se ligeiramente mais poderoso que os testes que usam as estatísticas $\widehat{R}^{*} \mathrm{e}$ $\widetilde{R}^{*}$. Podemos notar também que o teste baseado na estatística $\widetilde{R}^{*}$ mostrou-se mais poderoso que o teste baseado na estatística $\widehat{R}^{*}$. As demais Tabelas 4.14, 4.15 e 4.16 apresentam os poderes dos testes para os casos em que o tamanho amostral é $n=20$, a dimensão do vetor $\beta$ é $p=4$ e as covariáveis são geradas segundo as distribuições 
$t_{3}, t_{10}$ e $\chi_{5}^{2}$, respectivamente. Observa-se que mesmo gerando as covariáveis de outras distribuições os testes baseados nas estatísticas $\bar{R}^{*}$ e $\widetilde{R}^{*}$ são mais poderosos que o teste baseado na estatística $\widehat{R}^{*}$. Em geral, verificamos que os testes baseados nas estatísticas $\bar{R}^{*}$ e $\widetilde{R}^{*}$ apresentaram, em termos de poder, melhores desempenhos e, além disso, seus resultados foram muito parecidos.

Tabela 4.13: Poder dos Testes no modelo normal não linear (4.2), para $p=2,5$ e $8, n=20$ e covariáveis com distribuição uniforme.

\begin{tabular}{cccccccc}
\hline & & \multicolumn{6}{c}{$\psi$} \\
\cline { 2 - 7 }$p$ & Estatística & 0,2 & 0,6 & 1,0 & 1,4 & 1,8 & 2,2 \\
\hline \hline 2 & $\bar{R}^{*}$ & 10,32 & 34,77 & 81,93 & 99,60 & 100,00 & 100,00 \\
& $\widehat{R}^{*}$ & 9,19 & 32,40 & 79,07 & 99,44 & 100,00 & 100,00 \\
& $\widetilde{R}^{*}$ & 10,21 & 33,78 & 79,41 & 99,46 & 100,00 & 100,00 \\
\hline 5 & $\widetilde{R}^{*}$ & 8,81 & 23,04 & 58,69 & 94,97 & 100,00 & 100,00 \\
& $\widehat{R}^{*}$ & 6,66 & 17,23 & 46,03 & 87,06 & 99,70 & 100,00 \\
& $\widetilde{R}^{*}$ & 8,96 & 23,85 & 57,50 & 94,78 & 100,00 & 100,00 \\
\hline 8 & $\bar{R}^{*}$ & 8,14 & 17,48 & 40,54 & 79,19 & 99,39 & 100,00 \\
& $\widehat{R}^{*}$ & 6,92 & 13,87 & 31,26 & 65,09 & 95,62 & 99,97 \\
& $\widetilde{R}^{*}$ & 8,80 & 18,80 & 40,13 & 79,37 & 99,29 & 100,00 \\
\hline \hline
\end{tabular}


Tabela 4.14: Poder dos testes no modelo normal não linear (4.2), para $p=4, n=20$ e covariáveis com distribuição $t_{3}$.

\begin{tabular}{cccccccc}
\hline \hline & & \multicolumn{6}{c}{$\psi$} \\
\cline { 3 - 8 } Dist. & Estatística & 0,1 & 0,2 & 0,3 & 0,4 & 0,5 & 0,6 \\
\hline \hline \multirow{2}{*}{$t_{3}$} & $\widetilde{R}^{*}$ & 13,06 & 28,30 & 53,77 & 82,24 & 97,54 & 99,93 \\
& $\widehat{R}^{*}$ & 11,12 & 24,49 & 46,89 & 75,77 & 95,37 & 99,82 \\
& $\widetilde{R}^{*}$ & 13,03 & 28,35 & 52,11 & 80,08 & 96,75 & 99,88 \\
\hline \hline
\end{tabular}

Tabela 4.15: Poder dos testes no modelo normal não linear (4.2),

\begin{tabular}{cccccccc}
\multicolumn{6}{c}{ para $p=4, n=20$ e covariáveis com distribuição $t_{10}}$. \\
\hline \hline & & \multicolumn{7}{c}{$\psi$} \\
\cline { 3 - 8 } Dist. & Estatística & 0,5 & 0,7 & 0,9 & 1,1 & 1,3 & 1,5 \\
\hline \hline \multirow{2}{*}{$t_{10}$} & $\bar{R}^{*}$ & 43,90 & 66,17 & 85,19 & 95,91 & 99,28 & 99,96 \\
& $\widehat{R}^{*}$ & 38,99 & 59,74 & 79,83 & 92,85 & 98,07 & 99,63 \\
& $\widetilde{R}^{*}$ & 43,11 & 65,15 & 84,08 & 95,32 & 99,18 & 99,94 \\
\hline \hline
\end{tabular}

Tabela 4.16: Poder dos testes no modelo normal não linear (4.2),

\begin{tabular}{cccccccc}
\multicolumn{8}{c}{ para $p=4, n=20$ e covariáveis com distribuição $\chi_{5}^{2}}$. \\
\cline { 3 - 8 } Dist. & Estatística & 0,06 & 0,08 & 0,10 & 0,12 & 0,14 & 0,16 \\
\hline \hline \multirow{2}{*}{$\chi_{5}^{2}$} & $\bar{R}^{*}$ & 25,98 & 45,45 & 69,30 & 90,30 & 98,88 & 100,00 \\
& $\widehat{R}^{*}$ & 20,14 & 35,53 & 57,66 & 80,89 & 94,97 & 99,42 \\
& $\widetilde{R}^{*}$ & 25,93 & 45,36 & 67,73 & 89,47 & 98,74 & 99,97 \\
\hline \hline
\end{tabular}

Finalmente, apresentaremos os poderes dos testes baseados nas estatísticas ajustadas $\widetilde{R}^{*}, \widehat{R}^{*}$ e $\widetilde{R}^{*}$, utilizando o modelo normal não linear (4.3). 
Tabela 4.17: Poder dos testes no modelo normal não linear (4.3), para $n=10, p=4$ e covariáveis com distribuição uniforme.

\begin{tabular}{ccccccc}
\hline & \multicolumn{6}{c}{$\psi$} \\
\cline { 2 - 7 } Estatística & 0,0002 & 0,0012 & 0,0022 & 0,0032 & 0,0042 & 0,0052 \\
\hline \hline$\widetilde{R}^{*}$ & 9,56 & 43,56 & 82,65 & 97,50 & 99,82 & 99,99 \\
$\widehat{R}^{*}$ & 10,63 & 37,33 & 70,07 & 88,98 & 96,41 & 98,94 \\
$\widetilde{R}^{*}$ & 11,02 & 43,91 & 80,99 & 96,50 & 99,59 & 99,98 \\
\hline \hline
\end{tabular}

Tabela 4.18: Poder dos testes no modelo normal não linear (4.3), para $n=20, p=8$ e covariáveis com distribuição uniforme.

\begin{tabular}{ccccccc}
\hline & \multicolumn{6}{c}{$\psi$} \\
\cline { 2 - 7 } Estatística & 0,0004 & 0,0010 & 0,0016 & 0,0022 & 0,0028 & 0,0034 \\
\hline \hline $\bar{R}^{*}$ & 16,76 & 44,85 & 74,18 & 91,76 & 98,62 & 99,79 \\
$\widehat{R}^{*}$ & 16,22 & 39,66 & 64,57 & 84,10 & 93,83 & 97,92 \\
$\widetilde{R}^{*}$ & 17,33 & 45,43 & 73,61 & 91,30 & 98,38 & 99,72 \\
\hline \hline
\end{tabular}

Tabela 4.19: Poder dos testes no modelo normal não linear (4.3), para $n=20, p=4$ e covariáveis com distribuição $t_{3}$.

\begin{tabular}{cccccccc}
\hline \hline & & \multicolumn{6}{c}{$\psi$} \\
\cline { 3 - 7 } Dist. & Estatística & 0,001 & 0,003 & 0,005 & 0,007 & 0,009 & 0,011 \\
\hline \hline \multirow{2}{*}{$t_{3}$} & $\tilde{R}^{*}$ & 14,69 & 46,77 & 80,45 & 96,55 & 99,73 & 100,00 \\
& $\widehat{R}^{*}$ & 12,60 & 41,02 & 72,99 & 92,36 & 98,46 & 99,84 \\
& $\widetilde{R}^{*}$ & 15,18 & 45,84 & 79,15 & 96,01 & 99,64 & 100,00 \\
\hline \hline
\end{tabular}


Tabela 4.20: Poder dos testes no modelo normal não linear (4.3), para $n=20, p=4$ e covariáveis com distribuição $t_{10}$.

\begin{tabular}{cccccccc}
\hline \hline & & \multicolumn{6}{c}{$\psi$} \\
\cline { 3 - 8 } Dist. & Estatística & 0,002 & 0,003 & 0,004 & 0,005 & 0,006 & 0,007 \\
\hline \hline \multirow{2}{*}{$t_{10}$} & $\bar{R}^{*}$ & 35,40 & 61,15 & 81,36 & 94,01 & 98,76 & 99,83 \\
& $\widehat{R}^{*}$ & 28,25 & 51,33 & 71,64 & 87,45 & 95,54 & 98,73 \\
& $\widetilde{R}^{*}$ & 34,87 & 59,79 & 80,04 & 92,95 & 98,40 & 99,76 \\
\hline \hline
\end{tabular}

Tabela 4.21: Poder dos testes no modelo normal não linear (4.3),

\begin{tabular}{ccccccccc}
\multicolumn{6}{c}{ para $n=20, p=4$ e covariáveis com distribuição $\chi_{5}^{2}}$. \\
\cline { 3 - 8 } Dist. & Estatística & 0,02 & 0,04 & 0,06 & 0,08 & 0,10 & 0,12 \\
\hline \hline \multirow{2}{*}{$\chi_{5}^{2}$} & $\bar{R}^{*}$ & 25,71 & 60,63 & 86,75 & 97,17 & 99,74 & 99,97 \\
& $\widehat{R}^{*}$ & 21,29 & 50,81 & 76,50 & 92,19 & 97,91 & 99,51 \\
& $\widetilde{R}^{*}$ & 25,40 & 59,19 & 85,34 & 96,75 & 99,71 & 99,96 \\
\hline \hline
\end{tabular}

Os resultados apresentados nas Tabelas $4.17-4.21$, como esperado, mostram que os testes baseados nas estatísticas $\widetilde{R}^{*}$ e $\widetilde{R}^{*}$ são mais poderosos que o teste que usa a estatística $\widehat{R}^{*}$.

Em linhas gerais, podemos concluir que a estatística da razão de verossimilhanças sinalizada ajustada proposta por Skovgaard (1996) e baseada em covariâncias, ou seja, $\vec{R}^{*}$, é a mais recomendável. 


\section{Aplicação a Dados Reais}

Nesse capítulo, apresentamos dois modelos de regressão normais não lineares aplicados a dois conjuntos de dados reais. Nosso objetivo é testar hipóteses acerca de um parâmetro de cada um dos modelos utilizando as estatísticas $R, \bar{R}^{*}, \widehat{R}^{*}$ e $\widetilde{R}^{*}$ dadas no Capítulo 3. Para a realização dos testes, utilizamos a linguagem de programação Ox (Doornik, 2001). Os conjuntos de dados reais considerados referem-se a um estudo de câncer de mama em mulheres e de crescimento de um certo tipo de feijoeiro, respectivamente.

\subsection{Câncer de Mama}

Yi, Wu e Liu (2002) utilizaram um modelo de regressão normal não linear para explicar a relação entre a quantidade de estrógenos não receptivos do câncer mamário $(x)$ e o resultado do tratamento endócrino $(y)$. Aqui, y é a porcentagem de estrógenos receptivos positivos que ainda restaram após o tratamento. O modelo utilizado é

$$
y_{i}=\frac{\nu_{1} x_{i}}{\nu_{2}+x_{i}}+\sigma \varepsilon_{i}
$$


com $\varepsilon_{i} \sim \mathrm{N}(0,1)$ e $i=1,2, \ldots, 10$, sendo $\varepsilon_{1}, \cdots, \varepsilon_{10}$ variáveis aleatórias independentes. A função não linear, média de $y_{i}$, foi apresentada no Capítulo 2 (ver expressão (2.4)). Além disso, os autores fizeram a implementação computacional da estatística da razão de verossimilhanças sinalizada usual $R$ e de sua versão ajustada baseada em covariâncias $\left(\bar{R}^{*}\right)$ proposta por Skovgaard (1996) e decreveram o algoritmo para a re-alização dos testes de hipóteses baseados nessas estatísticas sobre o parâmetro $\psi=\nu_{2}$ utilizando os dados da Tabela 5.1 e o software estatístico S-Plus. Na Tabela 5.1 são apresentados também os valores preditos $\widehat{y}$ e os resíduos $y-\widehat{y}$ obtidos do modelo ajustado com $\widehat{\nu}_{1}=44,36(1,02), \widehat{\nu}_{2}=241,05(18,84)$ e $\widehat{\sigma}=1,16(0,60)$. Os valores entre parênteses são os respectivos erros padrão e as estimativas dos parâmetros são obtidas por máxima verossimilhança. A Figura 5.1 mostra o gráfico do modelo ajustado e os pontos amostrais observados.

Tabela 5.1: Dados resultantes da análise de um hormônio receptor de câncer de mama em humanos.

\begin{tabular}{cccc}
\hline \hline$x$ & $y$ & Valores Preditos $(\widehat{y})$ & $y-\widehat{y}$ \\
\hline \hline 84,6 & 12,1 & 11,5 & 0,6 \\
83,9 & 12,5 & 11,5 & 1,1 \\
148,2 & 17,2 & 16,9 & 0,3 \\
147,8 & 16,7 & 16,9 & $-0,2$ \\
463,9 & 28,3 & 29,2 & $-0,9$ \\
463,8 & 26,9 & 29,2 & $-2,3$ \\
964,1 & 37,6 & 35,5 & 2,1 \\
967,6 & 35,8 & 35,5 & 0,3 \\
1925,0 & 38,5 & 39,4 & $-0,9$ \\
1900,0 & 39,9 & 39,4 & 0,5 \\
\hline \hline
\end{tabular}




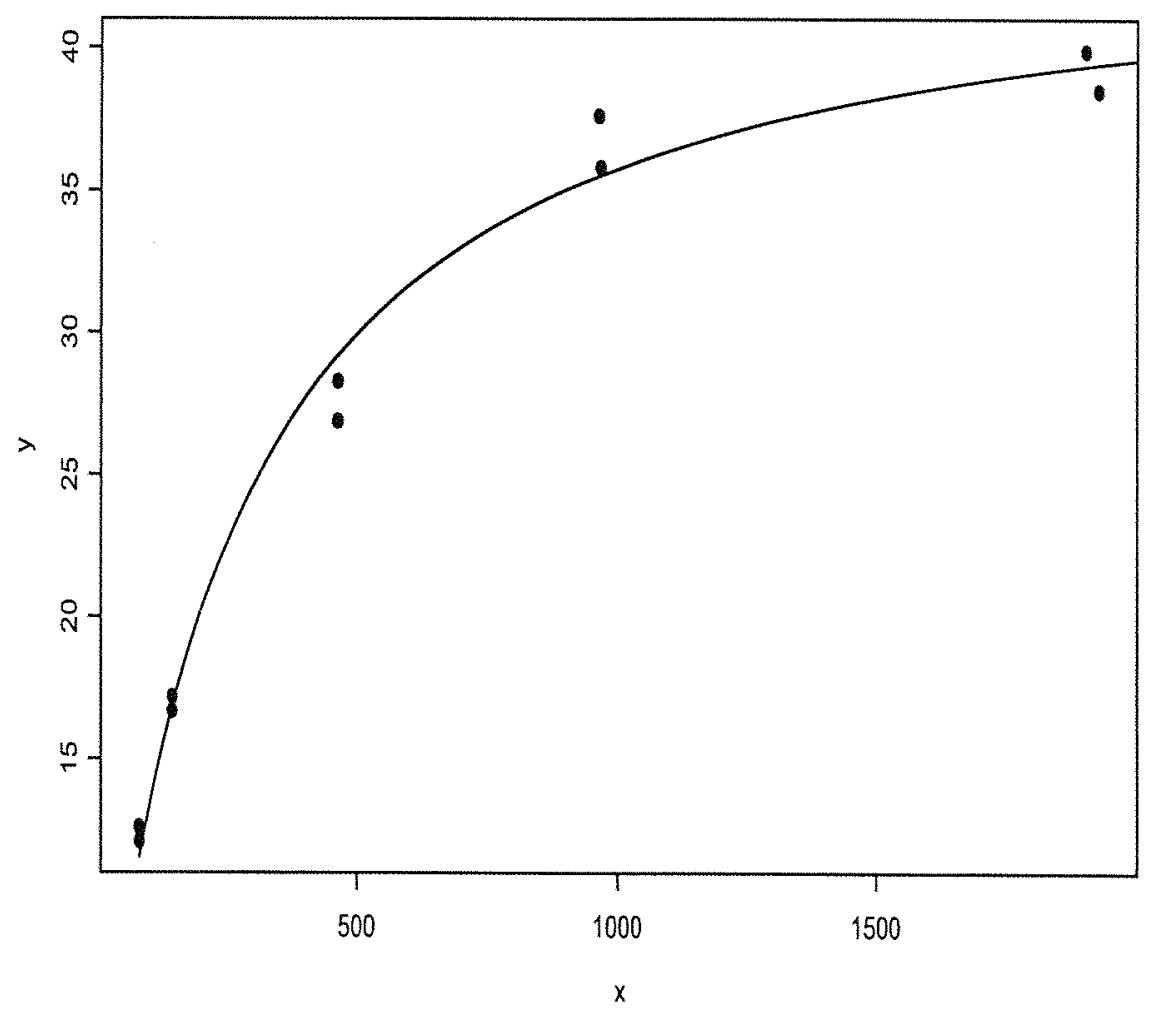

Figura 5.1: Gráfico do modelo de regressão não linear ajustado para os dados de câncer de mama.

Nosso objetivo aqui é obter os valores das estatísticas $R, \bar{R}^{*}, \widehat{R}^{*}$ e $\widetilde{R}^{*}$ utilizando o software de domínio público Ox (Doornik, 2001). No artigo de Yi, Wu e Liu (2002) foram implementados os testes baseados nas estatísticas $R$ e $\bar{R}^{*}$ e aqui, além desses testes, obtivemos os testes baseados em $\widehat{R}^{*}$ e $\widetilde{R}^{*}$, sendo essas a estatística da razão de verossimilhanças sinalizada modificada baseada em covariâncias empíricas proposta por Severini (1999) e a estatística baseada em uma estatística aproximadamente ancilar proposta por Fraser, Reid e Wu (1999), respectivamente.

Testamos a hipótese $\mathrm{H}_{0}: \psi \geq \psi_{0}$ contra $\mathrm{H}_{1}: \psi<\psi_{0}$. Yi, Wu e Liu (2002, 
p.783) construíram uma tabela com os níveis descritivos (" $p$ - values") dos testes para diferentes valores de $\psi_{0}$. A Tabela 5.1.1 segue a mesma idéia.

Tabela 5.1.1: Valores das estatisticas dos testes de hipóteses e os niveis descritivos dos testes entre parênteses.

\begin{tabular}{cccc}
\hline \hline$\psi_{0}$ & 260 & 280 & 300 \\
\hline$R$ & $-0,929(0,177)$ & $-1,737(0,041)$ & $-2,366(0,009)$ \\
$\widetilde{R}^{*}$ & $-0,954(0,170)$ & $-1,732(0,042)$ & $-2,303(0,011)$ \\
$\widehat{R}^{*}$ & $-0,825(0,205)$ & $-1,528(0,063)$ & $-2,074(0,019)$ \\
$\widetilde{R}^{*}$ & $-0,812(0,208)$ & $-1,521(0,064)$ & $-2,072(0,019)$ \\
\hline \hline
\end{tabular}

Considere o nível de significância de $5 \%$ e $\psi_{0}=260$. Nesse caso, as estatísticas $R, \bar{R}^{*}, \widehat{R}^{*}$ e $\widetilde{R}^{*}$ conduzem aos níveis descritivos $0,177,0,170,0,205$ e 0,208 , respectivamente, levando à não rejeição de $H_{0}$. Agora, suponha que desejamos testar as hipóteses considerando $\psi_{0}=280$. A hipótese nula é rejeitada apenas quando o teste é baseado na estatística da razão de verossimilhanças sinalizada usual ou quando se utiliza a estatística baseada em covariâncias $\left(\bar{R}^{*}\right)$. Mas se o interesse for testar as hipóteses quando $\psi_{0}=300$ a hipótese nula é rejeitada independentemente da estatística utilizada para realizar o teste.

O Capítulo 4 mostrou que o teste baseado na estatística $\bar{R}^{*}$ apresenta tamanhos empíricos próximos aos níveis nominais considerados e, além disso, é, em geral, o mais poderoso. Logo, os resultados baseados nessa estatística são os mais confiáveis. 


\subsection{Crescimento de Feijoeiros}

A Tabela 5.2 fornece o comprimento de feijoeiros $(y)$ e a quantidade de água na raiz (x), para uma amostra de 15 feijoeiros (Cordeiro e Paula, 1989). Além disso, acrescentamos os valores preditos $(\widehat{y})$ e os resíduos $(y-\widehat{y})$.

Tabela 5.2: Dados referentes ao crescimento de um certo tipo de feijoeiro.

\begin{tabular}{|c|c|c|c|}
\hline$x$ & $y$ & Valores Preditos $(\widehat{y})$ & $y-\widehat{y}$ \\
\hline 0,5 & 1,3 & 0,03 & 1,27 \\
\hline 1,5 & 1,3 & 0,23 & 1,07 \\
\hline 2,5 & 1,9 & 1,00 & 0,90 \\
\hline 3,5 & 3,4 & 2,72 & 0,68 \\
\hline 4,5 & 5,3 & 5,37 & $-0,07$ \\
\hline 5,5 & 7,1 & 8,51 & $-1,41$ \\
\hline 6,5 & 10,6 & 11,64 & $-1,04$ \\
\hline 7,5 & 16,0 & 14,39 & 1,61 \\
\hline 8,5 & 16,4 & 16,62 & $-0,22$ \\
\hline 9,5 & 18,3 & 18,32 & $-0,02$ \\
\hline 10,5 & 20,9 & 19,57 & 1,33 \\
\hline 11,5 & 20,5 & 20,47 & 0,03 \\
\hline 12,5 & 21,3 & 21,11 & 0,19 \\
\hline 13,5 & 21,2 & 21,55 & $-0,35$ \\
\hline 14,5 & 20,9 & 21,85 & $-0,95$ \\
\hline
\end{tabular}

Cordeiro e Paula (1989, p.189) utilizaram o modelo

$$
y_{i}=\nu_{1} \exp \left\{-\exp \left(\nu_{2}-\nu_{3} x_{i}\right)\right\}+\sigma \varepsilon_{i}
$$


com $\varepsilon_{i} \sim \mathrm{N}(0,1)$, para $i=1, \cdots, 15$ e $\varepsilon_{1}, \cdots, \varepsilon_{15}$ sendo variáveis aleatórias independentes, para explicar a relação entre $y$ e $x$. A função não linear, média de $y_{i}$, foi apresentada no Capítulo 2 (ver expressão (2.5)). As estimativas de máxima verossimilhança dos parâmetros são $\widehat{\nu}_{1}=22,51(0,75), \widehat{\nu}_{2}=2,11(0,21), \widehat{\nu}_{3}=0,39(0,04)$ e $\widehat{\sigma}=0,92(0,31)$. Os valores entre parênteses são os respectivos erros padrão. A Figura 5.2 mostra o gráfico do modelo ajustado e os pontos amostrais observados.

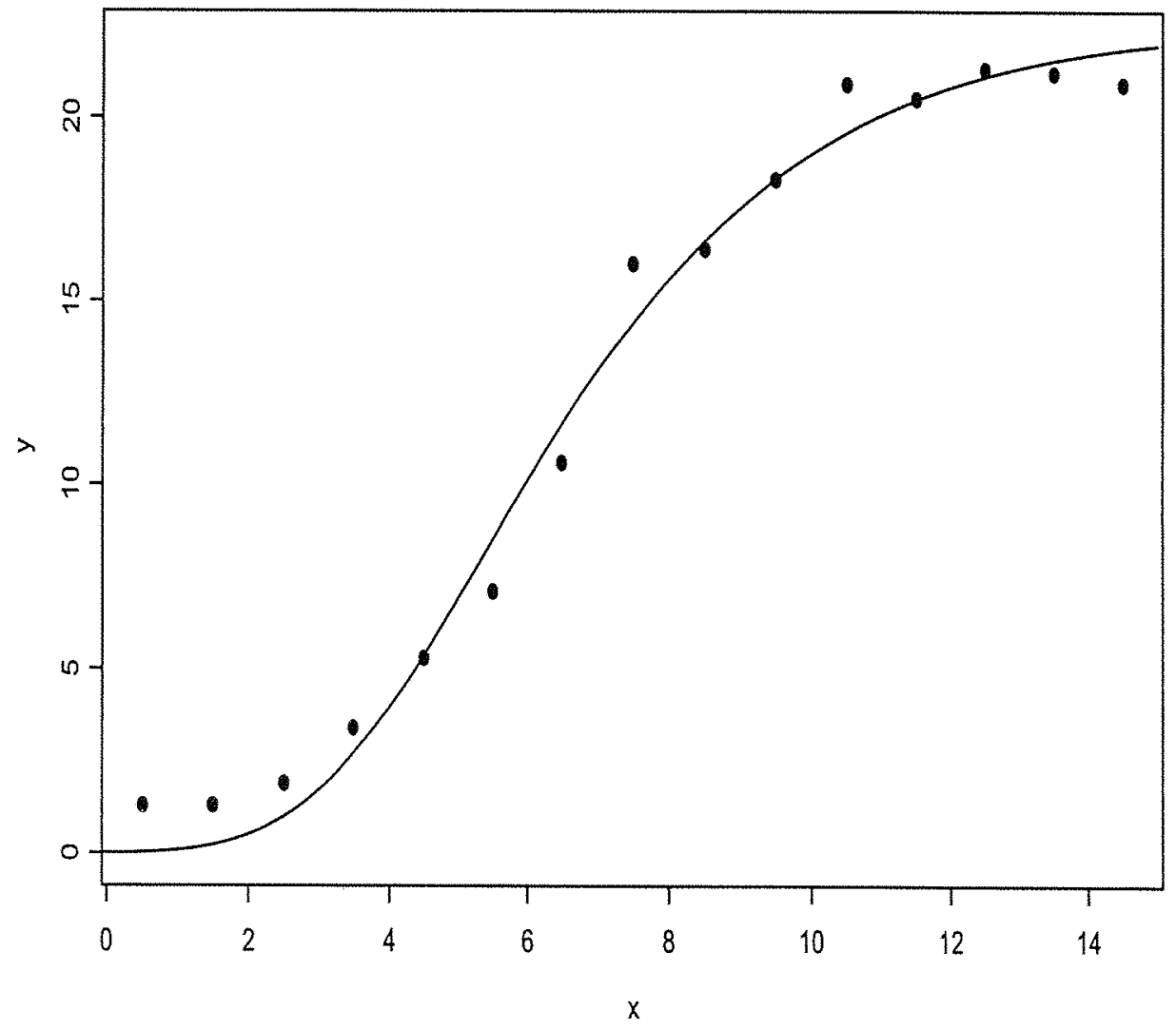

Figura 5.2: Gráfico do modelo de regressão não linear ajustado para os dados de crescimento de feijoeiros.

Os autores apresentaram o processo iterativo para estimar os parâmetros desse 
modelo e o gráfico dos valores ajustados e observados contra a quantidade de água na raiz da planta, para mostrar que esse modelo é adequado para esse conjunto de dados. Aqui, realizamos os testes baseados nas estatísticas $R, \widetilde{R}^{*}, \widehat{R}^{*}$ e $\widetilde{R}^{*}$ sobre o parâmetro $\psi=\nu_{3}$, utilizando a linguagem de programação $0 \mathrm{x}$. Testamos a hipótese $\mathrm{H}_{0}: \psi \leq \psi_{0}$ contra $\mathrm{H}_{1}: \psi>\psi_{0}$ com um nível de significância de $10 \%$. De maneira análoga à aplicação anterior, contruímos a Tabela 5.2.1.

Tabela 5.2.1: Valores das estatísticas dos testes de hipóteses e os niveis descritivos dos testes entre parênteses.

\begin{tabular}{cccc}
\hline \hline$\psi_{0}$ & $1 / 4$ & $1 / 3$ & $1 / 2$ \\
\hline$R$ & $3,150(0,001)$ & $1,295(0,098)$ & $-2,053(0,980)$ \\
$\widetilde{R}^{*}$ & $2,834(0,002)$ & $1,169(0,121)$ & $-1,907(0,972)$ \\
$\widehat{R}^{*}$ & $2,684(0,004)$ & $1,226(0,110)$ & $-1,524(0,936)$ \\
$\widetilde{R}^{*}$ & $2,744(0,003)$ & $1,115(0,132)$ & $-1,832(0,966)$ \\
\hline \hline
\end{tabular}

Para o nível de significância de $10 \%$ e supondo $\psi_{0}=1 / 4$, temos que $R, \bar{R}^{*}, \widehat{R}^{*} \mathrm{e}$ $\widetilde{R}^{*}$ conduzem aos respectivos níveis descritivos $0,001,0,002,0,004$ e 0,003 , levando à rejeição de $H_{0}$. Agora, considerando $\psi_{0}=1 / 3$, temos que $R, \vec{R}^{*}, \widehat{R}^{*}$ e $\widetilde{R}^{*}$ conduzem aos níveis descritivos $0,098,0,121,0,110$ e 0,132, respectivamente, ou seja, a hipótese nula é rejeitada quando o teste é baseado na estatística da razão de verossimilhanças sinalizada usual $(R)$ ao contrário do que ocorre quando o teste usa qualquer uma das estatísticas ajustadas. Mas se o interesse for testar as hipóteses quando $\psi_{0}=1 / 2$ a hipótese nula não é rejeitada, independentemente da estatística utilizada para realizar o teste. 
Capítulo 6

\section{Conclusões}

As simulações realizadas conduziram a diversas conclusões importantes a respeito do comportamento, em pequenas amostras, das estatísticas em estudo.

O estudo dos tamanhos dos testes indicou que o teste baseado na estatística da razão de verossimilhanças sinalizada $(R)$ é, em geral, liberal, conduzindo a taxas de rejeição da hipótese nula erroneamente com uma probabilidade maior que os níveis nominais considerados. Os testes baseados nas estatísticas da razão de verossimilhanças sinalizada modificadas fornecem taxas de rejeição observadas mais próximas dos niveis nominais considerados.

Com o intuito de verificar o impacto que o tamanho amostral pode causar na aproximação assintótica da distribuição das estatísticas para a distribuição normal padrão, foi realizado o estudo de simulação em que foi fixada a dimensão do vetor $\beta$ em $p=4$, as covariáveis geradas da distribuição uniforme e variado o tamanho amostral em $n=10,20$ e 30 . Notamos que conforme cresce o tamanho amostral, os tamanhos empíricos dos testes baseados na estatística original e nas ajustadas vão se aproximando dos níveis nominais considerados. Assim, os ajustes à estatística $R$ são mais necessários para tamanhos amostrais pequenos, como era esperado. 
Outro interesse foi verificar o impacto que o número de parâmetros de perturbação pode causar na aproximação assintótica das distribuições das estatísticas pela distribuição normal padrão. Neste caso, fixamos o tamanho amostral em $n=20$, as covariáveis foram geradas da distribuição uniforme e a dimensão do vetor $\beta$ variada em $p=2,5$ e 8 . Observamos que conforme se diminui a dimensão do vetor $\beta$, os tamanhos empíricos vão se aproximando dos níveis nominais considerados. Para uma dimensão maior do vetor $\beta$ a aproximação para a distribuição normal padrão da estatística $R$ é deteriorada. Logo, os testes baseados nas estatísticas ajustadas são mais necessários para os casos em que o número de parâmetros de perturbação for alto.

Finalmente, verificamos também o impacto que outros tipos de distribuições para as covariáveis podem causar nos desempenhos dos testes considerados. Para tanto, fixamos o tamanho amostral em $n=20$, a dimensão do vetor $\beta$ em $p=4$ e geramos covariáveis de distribuições que possuem caudas pesadas ou são assimétricas $\left(t_{3}, t_{10}\right.$ e $\left.\chi_{5}^{2}\right)$. Novamente os testes que usavam estatísticas ajustadas apresentaram melhores desempenhos que o teste baseado na estatística $R$.

Nas simulações sobre o poder dos testes, o teste baseado na estatística $\bar{R}^{*}$ mostrouse mais poderoso. O teste baseado na estatística $\widetilde{R}^{*}$ também apresentou um alto poder, mas em alguns casos perdeu, em termos de poder, para o teste baseado em $\tilde{R}^{*}$. No entanto, o teste baseado na estatística $\widehat{R}^{*}$ apresentou menor poder.

Em geral, nos estudos de simulação, destacou-se com melhor desempenho a estatística baseada em covariâncias $\left(\bar{R}^{*}\right)$, proposta por Skovgaard (1996), por fornecer testes com tamanhos empíricos próximos dos níveis nominais considerados e com maior poder do que os demais testes. Consequentemente, para as aplicações consideradas no Capítulo 5, consideram-se "mais confiáveis"as conclusões dos testes de hipóteses baseados nessa estatística. 
APÊNDICE A

\section{Programas}

Apresentamos nesse apêndice um programa desenvolvido na linguagem de programação Ox e sua saída. O programa foi utilizado para a realização de testes de hipóteses na aplicação referente ao estudo de câncer de mama em mulheres no Capítulo 5.

Os programas de simulação encontram-se em http://www. ime .usp.br/ daniest.

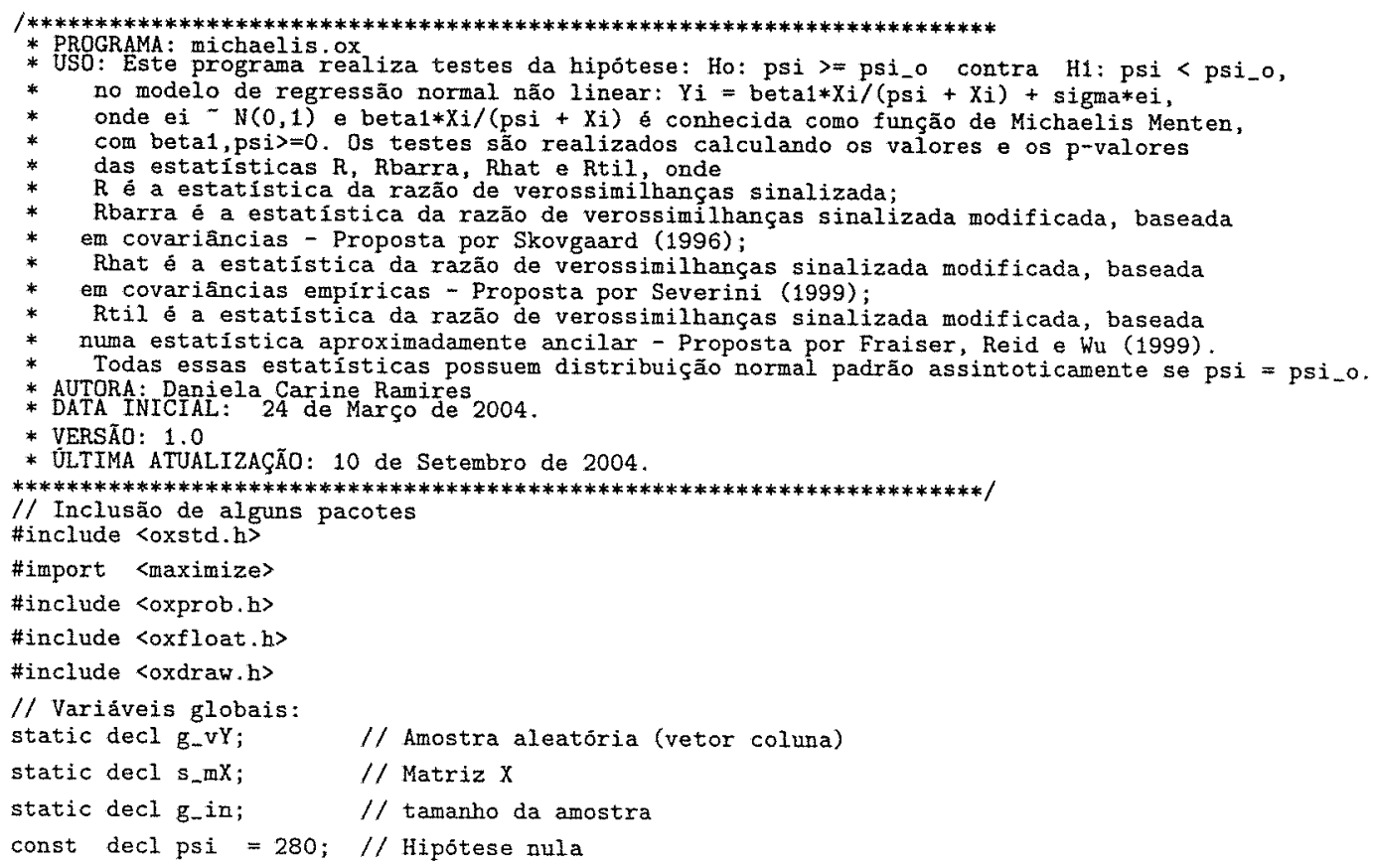




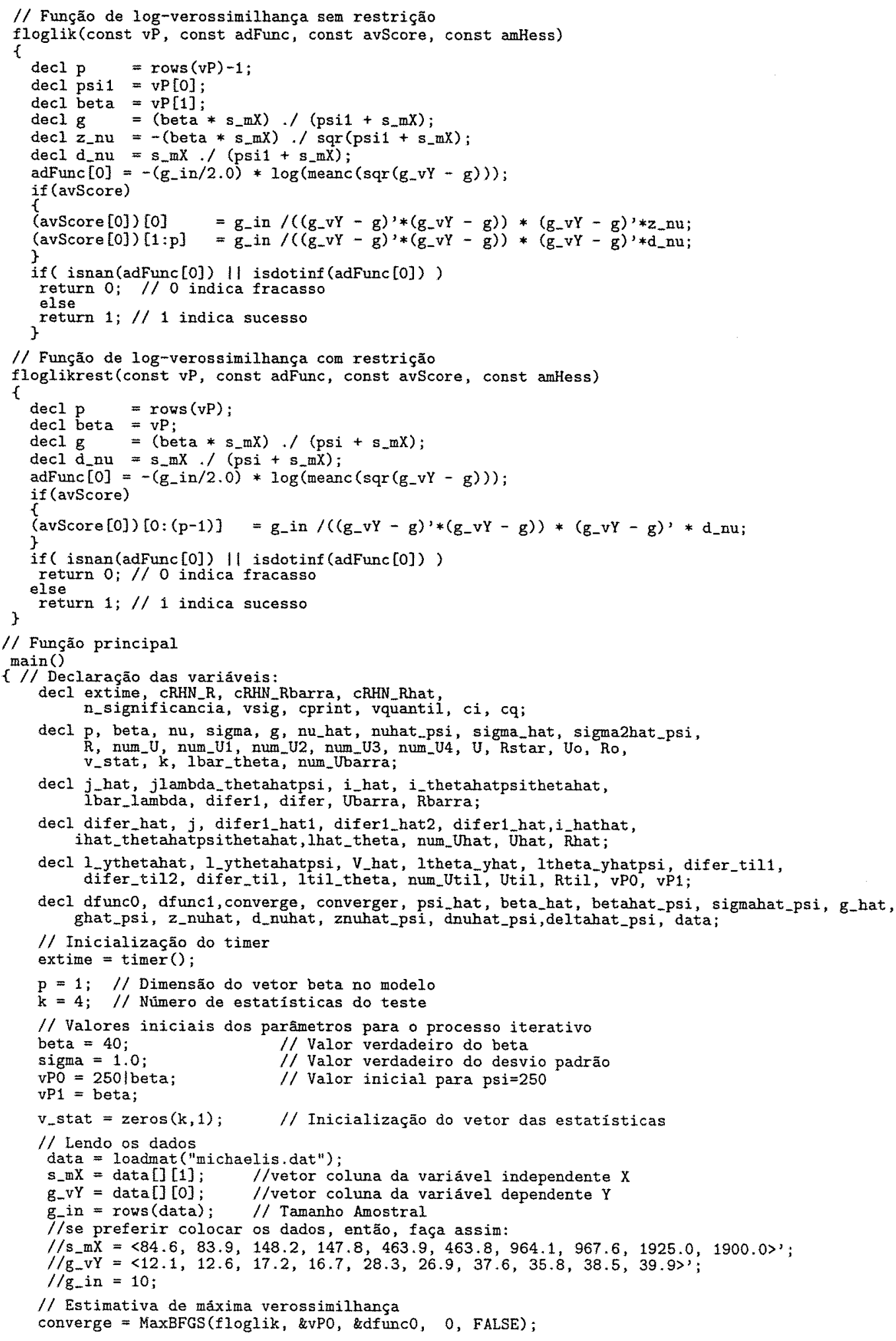




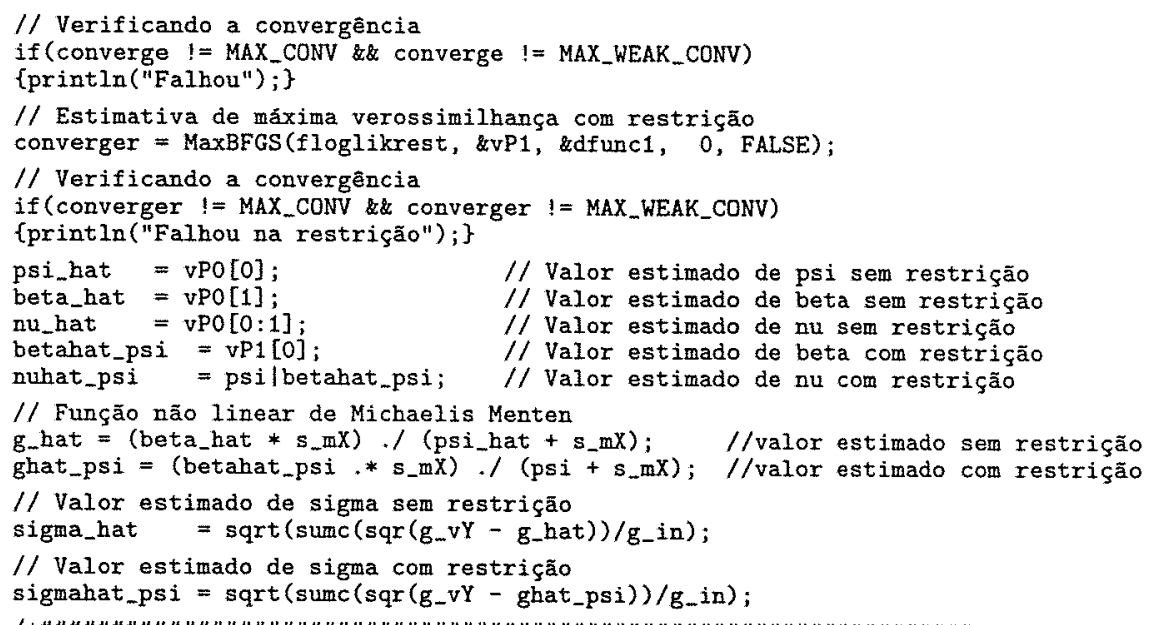

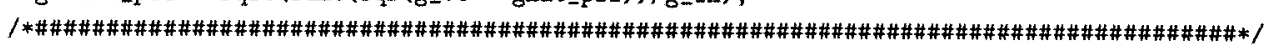

$/ / / / / / / / / / / / / / / / / /$ Estatistica da Razão de Veros. Sinalizada $\mathrm{R} / / / / / / / / / / / / / / / / / / / / / / / /$

// Log Verossimilhança avaliada em lhat theta

decl logver_hat $=-g_{-}$in $*\left(1+0.5 * \log \left(\operatorname{sqr}\left(\operatorname{sigma\_ hat}\right)\right)\right)$;

$/ /$ Log Verossimilhança avaliada em \hat \theta $\backslash p s i$

decl logverhat_psi $=-g_{-}$in $*(1+0.5 * \log ($ sqr (sigmahat_psi $))$ )

$\mathrm{R}=(($ psi_hat $-\mathrm{psi}) /$ fabs $($ psi_hat - psi) $) * \operatorname{sqrt}(2.0 *($ logver_hat - logverhat_psi) $)$;

if $(-0.1<R$ \& $R<0.1)$ Rbarra=Rhat=Rtil=R;\}

else \{

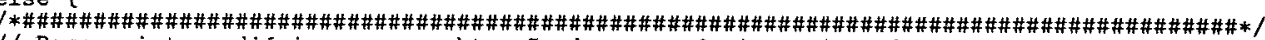

// Passos intermediários para a obtenção das estatisticas ajustadas:

$/ / z$ (nuhat) que é a derivada de $g$ (Função de Michaelis Menten) com relação a psi avaliada em nu_kat

$z_{\text {_nuhat }}=-$ (beta_hat $\left.* \mathrm{~s}_{-} \mathrm{mX}\right)$./ $\mathrm{sqr}\left(\mathrm{psi} \mathrm{i}_{\text {hat }}+\mathrm{s}_{-} \mathrm{mX}\right)$;

//d(nuhat) que é a derivada de $\mathrm{g}$ (Função de Michaelis Menten) com relação a beta avaliada em nu hat $d_{-}$nuhat $=s_{-} m X . /$ (psi_hat $\left.+s_{-} m X\right)$;

$/ / z$ (nuhat_psi) que é a derivada de g(Função de Michaelis Menten) com relação a psi avaliada //em nuhat_psi

znuhat_psi $=$ - (betahat_psi $\left.* s_{-} m X\right) . /$ sqr $\left(p s i+s_{-} m X\right)$;

//d(nuhat_psi) que é a derivada de g(Função de Michaelis Menten) com relação a beta avaliada

//em nuhat_psi

dnuhat_psi $=s_{-} \mathrm{mX}$./ (psi $+\mathrm{s}_{-} \mathrm{mX}$ )

deltahat_psi $=$ g_hat $_{-}$ghat_psi;

//zz(nuhat) que é a segunda derivada de g(Função de Michaelis Menten) com relação a psi־2

decl $z_{\text {Latat }}=\left(2.0 *\right.$ beta_hat $\left.* \mathrm{~s}_{-} \mathrm{mX}\right)$./ (psi_hat $\left.+\mathrm{s}_{-} \mathrm{mX}\right) \cdot \wedge(3.0)$;

//zd(nuhat) que é a segunda derivada de g(Função de Michaelis Menten) com relação a psi e //a beta avaliada em nuhat

decl $\mathrm{zd}_{-}$hat $=-1.0 *\left(\mathrm{~s}_{-} \mathrm{mX}\right) . / \mathrm{sqr}\left(\right.$ psi_hat $\left.+\mathrm{s}_{-} \mathrm{mX}\right)$;

// Denominador das U's:

1/ j_hat: matriz (p+2) $\mathrm{x}(\mathrm{p}+2)$

j_hat $=\left(1 /\right.$ sigma_hat $\left.{ }^{-2}\right) *\left(z_{-}\right.$nuhat ${ }^{\prime} * z_{-}$nuhat $-\left(g_{-}\right.$w $-g_{-}$hat $\left.), * z_{\text {_hat }}\right)-$

$\left(1 /\right.$ sigma_hat $\left.^{-2}\right) *\left(\left(z_{-}\right.\right.$nuhat' $\left.* d_{\text {_nuhat }}\right)-\left(g_{\text {_ }} \mathrm{vY} \text { - g_hat }\right)^{\prime} *$ zd_hat $)$

$(2 /$ sigma_hat -3$) *\left(g_{-} v Y-g_{-} \text {hat }\right)^{2} * z_{-}$nuhat

$(1 /$ sigma_hat -2$) *($ (z_nuhat' * d_nuhat $)-\left(g_{-} v Y-g_{-}\right.$hat $), *$ zd_hat $)$ -

(1.0/sigma_hat $\left.{ }^{-2}\right) *$ (d_nuhat, * d_nuhat)

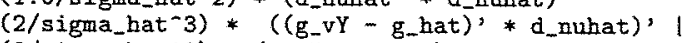

$(2 /$ sigma_hat 3$) *\left(g_{-} v \bar{Y}-g_{-}\right.$hat $\left.)\right)^{*} z_{\text {_nuhat }}$

$(2 /$ sigma hat -3$) *$ (g-vY - g hat) $) *$ d nuhat

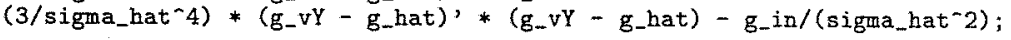

// jlambda_thetahatpsi: matriz $(p+1) \times(p+1)$

jlambda_thetahatpsi $=(1.0 /$ sigmahat psi-2) $*$ (dnuhat psi' $*$ dnuhat psi) -

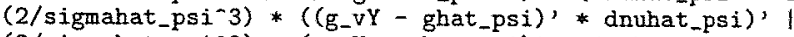

(2/sigmahat_psi $\left.{ }^{-3}\right) *\left(g_{-v Y}-g_{\text {ghat_psi) }}\right.$ * dnuhat_psi

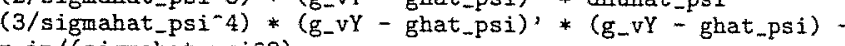

g_in/(sigwahat_psi-2);

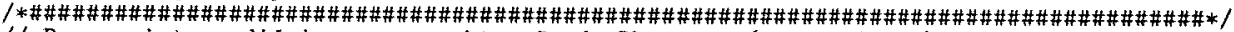

// Passos intermediários para a obtenção de Rbarra*: (ver p. 23-25)

// Passos intermediários para a obtenção do difer (equação 3.13 , p. 24)

//difer1: diferença entre os $Q$ 's (equação $3.13, p .24)$, matriz $1 x(p+2)$ 


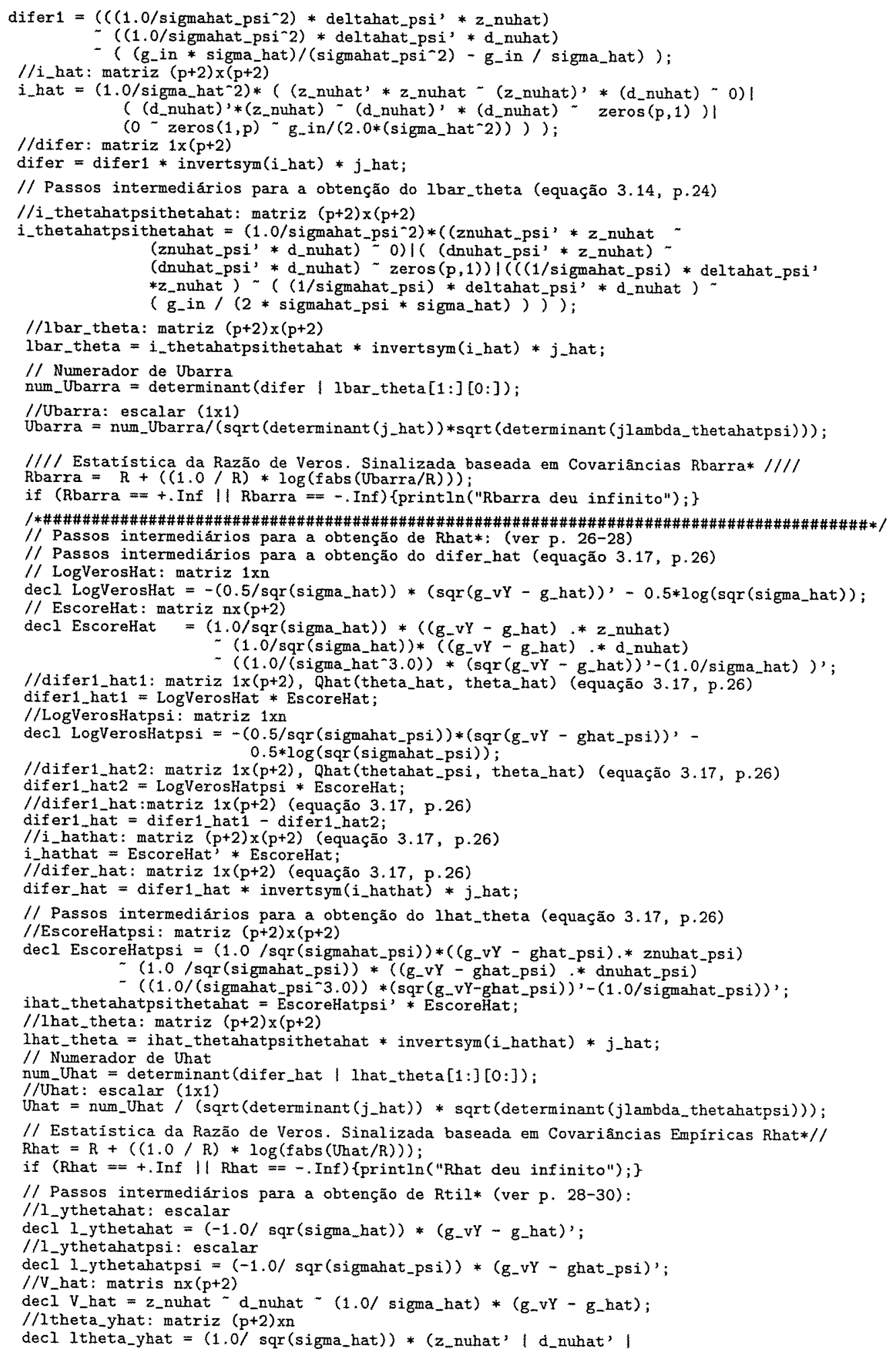




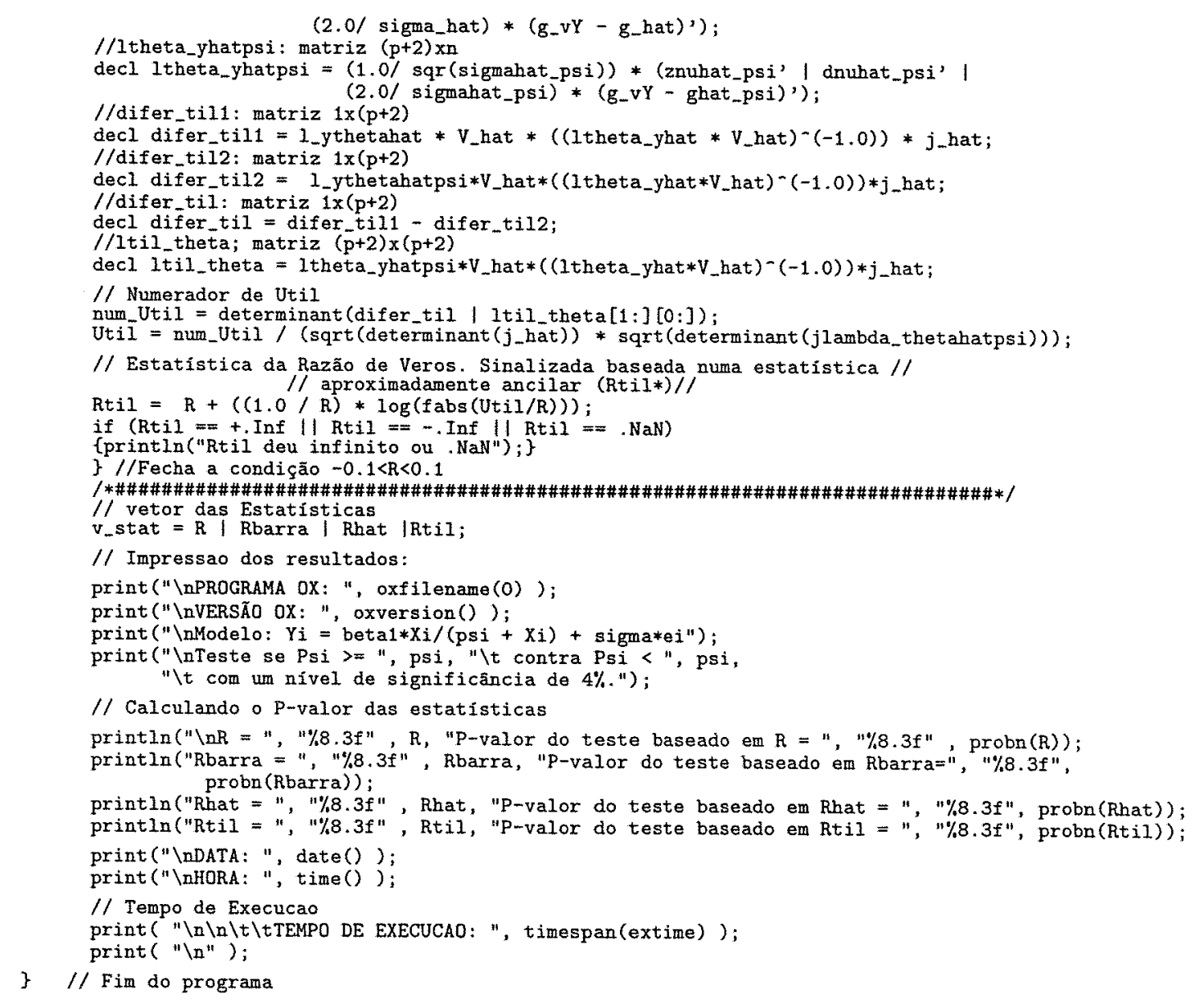

Saída:

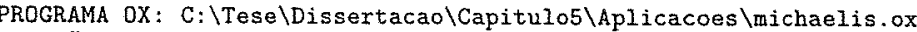

VERSÃO OX: 330

Modelo: $Y_{i} \equiv$ beta $1 * X_{i} /(p s i+X i)+$ sigma $*$ ei
Teste se Psi $>=280 \quad$ contra Psi $<280$ com un nivel de significancia de $4 \%$

$R=1.737$

P-vaior do teste baseado em $R=$

Rbarra $=$

P-vaior do teste baseado em Rbarra=

Rhat $=52$

P-vaior do teste baseado em Rhat $=$

R.til $\stackrel{0.063}{=}$

P-vaior do teste baseado em Rtil =

DATA: $20-09-2004$

TEMPO DE EXECUCAO: 0.00 


\section{Referências Bibliográficas}

[1] Barndorff-Nielsen, O. E. (1986). Inference on full and partial parameters based on the standardized signed log likelihood ratio. Biometrika, 73, 307-322.

[2] Barndorff-Nielsen, O. E. (1990). A note on the standardized signed log likelihood ratio. Scandinavian Journal of Statistics, 17, 157-160.

[3] Barndorff-Nielsen, O. E. (1991). Modified signed log likelihood ratio. Biometrika, 78, 557-563.

[4] Barndorff-Nielsen, O. E. e Cox, D. R. (1994). Inference and Asymptotics. London: Chapman and Hall.

[5] Barndorff-Nielsen, O. E. (1995). Stable and invariant adjusted profile likelihood and directed likelihood for curved exponential models. Biometrika, 82, 489-500.

[6] Cordeiro, G. M. (1992). Introdução à Teoria de Verossimilhança. $10^{0}$ Simpósio Nacional de Probabilidade e Estatística.

[7] Cordeiro, G. M. e Paula, G. A. (1989). Modelos de Regressão para Análise de Dados Univariados. $17^{\mathrm{O}}$ Colóquio Brasileiro de Matemática. Instituto de Matemática Pura e Aplicada do CNPq.

[8] DiCiccio, T. J. e Martin, M. A. (1993). Simple modifications for signed roots of likelihhod ratio statistics. Journal of the Royal Statistical Society, B, 55, 305-316. 
[9] Doornik, J. A. (2001). Ox: An Object-oriented Matrix Programming Language. 4th edition, London: Timberlake Consultants and Oxford: http://www.nuff.ox.ac.uk/Users/Doornik.

[10] Fraser, D. A. S. (1990). Tail probabilities from observed likelihoods. Biometrika, 77, 65-76.

[11] Fraser, D. A. S., Reid, N. e Wu, J. (1999). A simple formula for tail probabilities for frequentist and Bayesian inference. Biometrika, 86, 249-264.

[12] Jensen, J. L. (1992). The modified signed likelihood ratio and saddlepoint approximations. Biometrika, 79, 693-703.

[13] Jensen, J. L. (1993). A historical sketch and some new results on the improved log likelihood ratio statistic. Scandinavian Journal of Statistics, 1-15.

[14] Jensen, J. L. (1995), Saddlepoint Approximations. Oxford: Oxford University Press.

[15] Jensen, J. L. (1997). A simple derivation of $R^{*}$ for curved exponential families. Scandinavian Journal of Statistics, 24, 33-46.

[16] Larsen, P. V., Blæsild, P. e Sørensen, M. K. (2002). Improved likelihood ratio tests on the von Mises-Fisher distribution. Biometrika, 89, 4, 947-951.

[17] Nocedal, J. e Wright, S. J. (1999). Numerical Optimization. New York: SpringerVerlag.

[18] Rao, C. R. (1973). Linear Statistical Inference and its Applications. New York: John Wiley.

[19] Ratkowsky, D. A. (1983). Nonlinear Regression Modelling. New York: Marcel Dekker.

[20] Reid, N. (1996). Likelihood and higher-order appoximations to tails areas: a review and annotated bibliography. Canadian Journal of Statistics, 24, 141-166.

[21] Severini, T. A. (1999). An empirical adjustment to the likelihood ratio statistic. Biometrika, 86, 235-247.

[22] Severini, T. A. (2000). Likelihood Methods in Statistics. Oxford: University Press. 
[23] Skovgaard, I. M. (1996). An explicit large-deviation appoximation to one-parameter tests. Bernoulli, 2, 145-165.

[24] Skovgaard, I. M. (2001). Likelihood asymptotics. Scandinavian Journal of Statistics, $28,3-32$.

[25] Sweeting, T. J. (1995). Bayesian and likelihood appoximations. Biometrika, 82, 1-23.

[26] Yi, G. Y., Wu, J. e Liu, Y. (2002). Implementation of higher-order asymptotics to S-plus. Computational Statistics and Data Analysis, 40, 775-800. 